\title{
THE BOLOCAM GALACTIC PLANE SURVEY. XIV. PHYSICAL PROPERTIES OF MASSIVE STARLESS AND STAR-FORMING CLUMPS
}

\author{
Brian E. Svoboda ${ }^{1}$, Yancy L. Shirley ${ }^{1,2}$, Cara Battersby ${ }^{3}$, Erik W. Rosolowsky ${ }^{4}$, Adam G. Ginsburg ${ }^{5,6}$, \\ Timothy P. Ellsworth-Bowers ${ }^{6}$, Michele R. Pestalozzi ${ }^{7}$, Miranda K. Dunham ${ }^{8}$, Neal J. Evans II $^{9}$, \\ JOHN BALly ${ }^{6}$, AND JASON GLENN ${ }^{6}$ \\ ${ }^{1}$ Steward Observatory, University of Arizona, 933 North Cherry Avenue, Tucson, AZ 85721, USA; svobodb@email.arizona.edu \\ ${ }_{2}$ Adjunct Astronomer, National Radio Astronomy Observatory, USA \\ ${ }^{3}$ Department of Physics, 4-181 CCIS, University of Alberta, Edmonton AB T6G 2E1, Canada \\ ${ }^{4}$ CASA, University of Colorado, 389-UCB, Boulder, CO 80309, USA \\ ${ }^{5}$ European Southern Observatory, Karl-Schwarzschild-Strasse 2, D-85748 Garching bei München, Germany \\ ${ }^{6}$ University of Gothenburg, SE-412 96 Gothenburg, Sweden \\ ${ }^{7}$ Harvard-Smithsonian Center for Astrophysics, 60 Garden Street, Cambridge, MA 02138, USA \\ ${ }^{8}$ Department of Astronomy, Yale University, P.O. Box 208101, New Haven, CT 06520, USA
Department of Astronomy, The University of Texas at Austin, 2515 Speedway, Stop C1400, Austin, TX 78712-1205, USA \\ Received 2015 July 2; accepted 2015 November 25; published 2016 May 5
}

\begin{abstract}
We sort 4683 molecular clouds between $10^{\circ}<\ell<65^{\circ}$ from the Bolocam Galactic Plane Survey based on observational diagnostics of star formation activity: compact $70 \mu \mathrm{m}$ sources, mid-IR color-selected YSOs, $\mathrm{H}_{2} \mathrm{O}$ and $\mathrm{CH}_{3} \mathrm{OH}$ masers, and $\mathrm{UCH}$ II regions. We also present a combined $\mathrm{NH}_{3}$-derived gas kinetic temperature and $\mathrm{H}_{2} \mathrm{O}$ maser catalog for 1788 clumps from our own GBT $100 \mathrm{~m}$ observations and from the literature. We identify a subsample of $2223(47.5 \%)$ starless clump candidates (SCCs), the largest and most robust sample identified from a blind survey to date. Distributions of flux density, flux concentration, solid angle, kinetic temperature, column density, radius, and mass show strong ( $>1$ dex) progressions when sorted by star formation indicator. The median SCC is marginally subvirial $(\alpha \sim 0.7)$ with $>75 \%$ of clumps with known distance being gravitationally bound $(\alpha<2)$. These samples show a statistically significant increase in the median clump mass of $\Delta M \sim 170-370 M_{\odot}$ from the starless candidates to clumps associated with protostars. This trend could be due to (i) mass growth of the clumps at $\dot{M} \sim 200-440 M_{\odot} \mathrm{Myr}^{-1}$ for an average freefall $0.8 \mathrm{Myr}$ timescale, (ii) a systematic factor of two increase in dust opacity from starless to protostellar phases, and/or (iii) a variation in the ratio of starless to protostellar clump lifetime that scales as $\sim M^{-0.4}$. By comparing to the observed number of $\mathrm{CH}_{3} \mathrm{OH}$ maser containing clumps, we estimate the phase lifetime of massive $\left(M>10^{3} M_{\odot}\right)$ starless clumps to be $0.37 \pm 0.08 \mathrm{Myr}\left(M / 10^{3} M_{\odot}\right)^{-1}$; the majority $\left(M<450 M_{\odot}\right)$ have phase lifetimes longer than their average freefall time.
\end{abstract}

Key words: ISM: clouds - ISM: molecules - masers - stars: formation - submillimeter: ISM - surveys

Supporting material: machine-readable tables

\section{INTRODUCTION}

Massive stars $\left(M>8 M_{\odot}\right)$ strongly influence the evolution of galaxies and the interstellar medium (ISM), yet their formation remains an open problem in contemporary astrophysics (see reviews by McKee \& Ostriker 2007; Tan et al. 2014). A major bottleneck in the study of massive star formation is the difficulty in identifying and systematically analyzing the properties of the incipient phases of intermediateand high-mass (protocluster) star formation, high-mass starless clumps. These objects fragment into dense starless cores that subsequently contract to form individual or bound systems of protostars (Tan et al. 2014). Starless cores (and gravitationally bound prestellar cores) are cold $\left(T_{\mathrm{d}}<20 \mathrm{~K}\right)$, dense $\left(n\left(\mathrm{H}_{2}\right)>10^{4} \mathrm{~cm}^{-3}\right)$, and centrally concentrated objects that lack an embedded protostar (di Francesco et al. 2007). The study of low-mass $\left(<10 M_{\odot}\right)$ starless cores has received considerable attention owing to their proximity and the systematic mapping of nearby molecular clouds in the Gould Belt (Ward-Thompson et al. 1994; Tafalla et al. 2004; André et al. 2007, 2013). In contrast, the study of intermediateand high-mass starless cores and clumps is still in a nascent state owing in part to their greater distances and few systematic observations to identify them (Tackenberg et al. 2012; Wilcock et al. 2012; Ragan et al. 2013; Traficante et al. 2015a). Our understanding of both how cluster formation is initiated and the ensuing protocluster evolution ultimately depends on identifying and constraining the physical properties of representative samples of high-mass starless clumps. This situation is beginning to change with the publication of catalogs of 170 and 667 starless clumps identified with the Herschel Space Observatory toward Infrared Dark Clouds (IRDCs; Wilcock et al. 2012; Traficante et al. 2015a). In this paper, we analyze the star formation activity of clumps observed in the Bolocam Galactic Plane Survey (BGPS) to identify a population of over 2000 massive starless clump candidates (SCCs) in an $84.4 \mathrm{deg}^{2}$ region of the first quadrant of the Galaxy.

Recent blind surveys of the dust continuum emission at (sub) millimeter wavelengths are able to detect embedded starforming regions in a wide range of evolutionary states, including the starless phases (e.g., Tackenberg et al. 2012; Traficante et al. 2015a), throughout the Galaxy. There are three primary surveys that have mapped far-infrared through 
millimeter wavelength emission in the Milky Way. The BGPS $^{10}$ (Rosolowsky et al. 2010; Aguirre et al. 2011; Ginsburg et al. 2013) at $\lambda=1.1 \mathrm{~mm}$ surveyed in the range $-10^{\circ}<\ell<90^{\circ}$ and targeted regions in the second and third quadrants with $|b|<0.5^{\circ}$ (expands to $|b|<1.5$ at selected $\ell$ ) at $33^{\prime \prime}$ resolution. ATLASGAL (Schuller et al. 2009; Contreras et al. 2013; Csengeri et al. 2014) surveyed at $\lambda=870 \mu \mathrm{m}$ in the range $-60^{\circ}<\ell<60^{\circ}$ and $|b|<1.5$ with an extension covering $-80^{\circ}<\ell<-60^{\circ}$ and $-2.0<b<+1.0$ at $22^{\prime \prime}$ resolution. In the far-infrared and submillimeter, the Herschel Infrared Galactic Plane Survey (Hi-GAL; Molinari et al. 2010) surveyed the entire Galactic plane in the five PACS and SPIRE bands from $\lambda=60$ to $500 \mu \mathrm{m}$. In this paper, we shall focus on studying the physical properties of starless and star-forming clumps identified in the BGPS.

The BGPS identified 8294 clumps with $1.1 \mathrm{~mm}$ flux densities above $100 \mathrm{mJy}$ and 8594 clumps cataloged in total (Rosolowsky et al. 2010; Aguirre et al. 2011; Ginsburg et al. 2013). The second data release (v2.0) of the Bolocam images and source catalog (Ginsburg et al. 2013) used improved analysis of the time-stream data to improve angular flux recovery with full recovery out to $\sim 80^{\prime \prime}$ and partial recovery out to $\sim 300^{\prime \prime}$. The v2.0 data also resolve systematic flux and pointing calibration offsets and include new fields in the second quadrant. In this work we exclusively use the BGPS v2.0 data products, and all clumps are referenced to the BGPS v2.0 maps and Bolocat clumps.

Extensive follow-up observations of BGPS clumps have been carried out to determine unique velocities, kinematic distances, and gas kinetic temperatures to use in calculations of physical properties. Follow-up spectroscopic observations of the BGPS catalog in dense molecular gas tracers $\mathrm{HCO}^{+} 3-2$ and $\mathrm{N}_{2} \mathrm{H}^{+} 3-2$ with the Arizona Radio Observatory's Heinrich Hertz Submillimeter Telescope (HHSMT; Schlingman et al. 2011; Shirley et al. 2013) detected about half of the objects in the BGPS catalog ( $>97 \%$ of those clumps have a single velocity component) from which kinematic distances may be derived. A novel Bayesian technique has been developed to calculate a continuous function of the probability that a source lies at a given heliocentric distance (EllsworthBowers et al. 2013, 2015). The posterior distance probability density function (DPDF) is constructed by multiplying the bimodal likelihood function (with equal probability of being located at the near or far kinematic distance) with prior distributions that account for the source positions relative to the known $\mathrm{H}_{2}$ distribution in the Galaxy and the coincidence and contrast of $8 \mu \mathrm{m}$ absorption features (Ellsworth-Bowers et al. 2013). Since the dense molecular gas lines are only detected toward approximately half of BGPS clumps, Ellsworth-Bowers et al. (2015) extended the number of sources with bimodal likelihood functions by developing a morphological matching technique between ${ }^{13} \mathrm{CO} 1-0$ from the Galactic Ring Survey (GRS; Jackson et al. 2006) and the BGPS $1.1 \mathrm{~mm}$ emission. This technique assigns a clump the dominant ${ }^{13} \mathrm{CO}$ velocity component that matches the $1.1 \mathrm{~mm}$ morphology. The DPDF formalism is a powerful tool that permits proper accounting and propagation of the distance uncertainty in calculations of distance-dependent physical properties (i.e., size, mass, and luminosity).

\footnotetext{
${ }^{10}$ See http://irsa.ipac.caltech.edu/data/BOLOCAM_GPS/
}

Early BGPS papers have characterized clump physical properties on modest subsets of the BGPS. Schlingman et al. (2011) determined the physical properties of 529 sources drawn uniformly from the BGPS $1.1 \mathrm{~mm}$ flux distribution, finding that BGPS sources have median physical radii of $0.75 \mathrm{pc}$, median total masses of $330 M_{\odot}$, and nonthermal line widths that are 10 times the thermal line width on median. Dunham et al. (2011b) performed an initial evolutionary analysis on a subsample of 456 BGPS sources toward which $\mathrm{NH}_{3}$ was detected, finding a median gas kinetic temperature of $16 \mathrm{~K}$ and identifying a subset of SCCs that composes $\sim 1 / 3$ of their sample. While BGPS sources are commonly referred to generically as clumps, Dunham et al. (2011b) pointed out that BGPS objects are part of a continuum of hierarchical structure that samples cores, clumps, and clouds on different scales and at different distances. Indeed, higherresolution observations at $350 \mu$ m indicate that BGPS clumps typically break up into collections of smaller substructures (Merello et al. 2015). All of these early studies made crude assumptions to resolve the kinematic distance ambiguity (KDA; i.e., all sources associated with $8 \mu \mathrm{m}$ absorption are at the near distance or using the presence or lack of $\mathrm{H}$ I self-absorption to distinguish between near and far kinematic distance; see Section 4.3). Ellsworth-Bowers et al. (2015) is the first study to utilize the full power of the DPDF formalism, in which they characterize the clump mass distribution for the subset of 1710 clumps with well-constrained DPDFs. They find that the clump mass distribution is best fit with a lognormal distribution with an approximately power-law distribution for high-mass sources and a power-law index that is intermediate between that observed for giant molecular clouds (GMCs) and that observed for the stellar initial mass function (IMF).

A significant remaining challenge with the BGPS data set is to identify the star formation activity of all of the BGPS clumps and, in particular, identify the earliest phase of protocluster formation: starless clumps. The extensive characterization and follow-up of the BGPS presents an excellent sample drawn from a blind survey to rigorously calculate population statistics and physical properties. Not only will measuring the physical properties of starless clumps constrain the initial conditions of protocluster evolution, but starless clumps will also be the proving grounds between theories of high-mass star formation, such as virialized turbulent core accretion (TCA; McKee \& Tan 2003) and a subvirial competitive accretion (CA; Bonnell et al. 2001, 2007, p. 149). Resolving the current tension between these two theories is a major objective in the near-term study of high-mass star formation, but it requires the identification of a representative sample of massive starless clumps for further high-resolution study. In this paper we address questions related to how starless clumps, as the host environments of future intermediate- and high-mass stars, evolve through the starless phase into the protostellar phase. How long does the starless phase last, and how does the starless clump lifetime depend on the mass of the clump? Do clumps undergo significant evolution in their fundamental physical properties, and do they undergo significant interaction with the surrounding molecular cloud throughout their lifetimes? We study these questions by analyzing large, statistical subsamples $\left(N \sim 10^{2}-10^{3}\right)$ of BGPS clumps.

We present a catalog of 4683 BGPS clumps sorted by observational indicators of star formation activity from a comparison to other Galactic plane surveys in a common overlap range of $10^{\circ}<\ell<65^{\circ}$. We also present 1215 targeted 


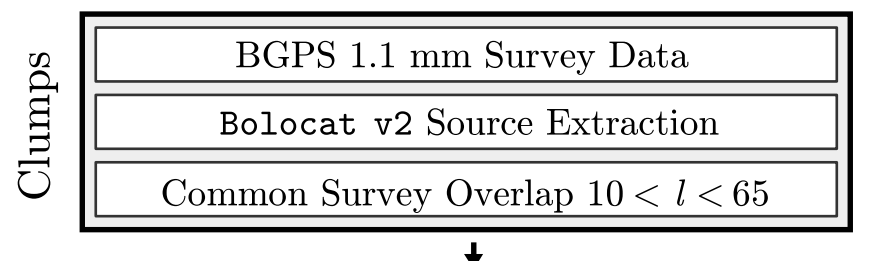

\begin{tabular}{|c|c|}
\hline \multicolumn{2}{|c|}{ Starless Clump Candidates } \\
\hline \multicolumn{2}{|c|}{ Protostellar Clumps } \\
\hline \multicolumn{2}{|c|}{ HG70 Uniq. : Hi-GAL 70 um Unique } \\
\hline Mid. IR & : 2-24 um YSO \\
\hline $\mathrm{H}_{2} \mathrm{O}$ & : Water Maser \\
\hline $\mathrm{CH}_{3} \mathrm{OH}$ & : Methanol Maser \\
\hline UCHII & : Ultra-Compact HII Reg. \\
\hline
\end{tabular}

$\downarrow$

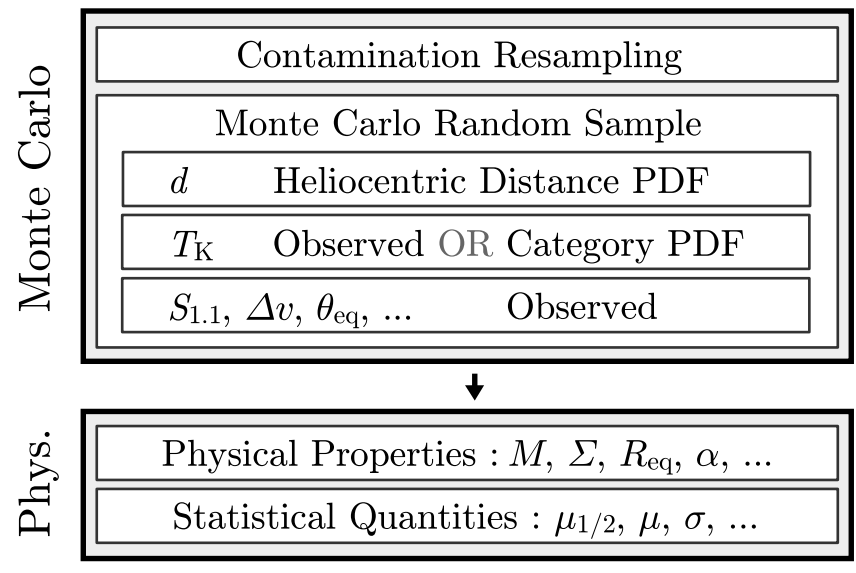

Figure 1. Flow chart describing the BGPS catalog, sorting by star formation indicator, Monte Carlo random sampling, and property calculation.

$\mathrm{NH}_{3}$ and $\mathrm{H}_{2} \mathrm{O}$ maser observations with the $100 \mathrm{~m}$ Robert $\mathrm{C}$. Byrd Green Bank Telescope (GBT) in Section 2. Schematically, we follow the procedure shown in Figure 1. In Section 3 we describe the survey data sets and methods in developing a BGPS star formation catalog. In Section 4 we describe the methods used to extend the sample of DPDFs to additional sources with a position-position-velocity (PPV) clustering algorithm. We use Monte Carlo (MC) sampling of the DPDFs, resampling techniques to account for contamination of evolved stars, and sampling of uncertainties on observed quantities to calculate and analyze the physical properties of the BGPS clumps associated with star formation indicators in Section 5. We discuss the properties of the SCC sample and possible evidence for clump mass growth during this phase in Section 6.

\section{2. $\mathrm{NH}_{3}$ AND $\mathrm{H}_{2} \mathrm{O}$ MASER SURVEY OF BGPS SOURCES}

\subsection{New GBT $\mathrm{NH}_{3}$ Observations}

We have conducted targeted, spectroscopic observations of the $\mathrm{NH}_{3}(1,1),(2,2)$, and $(3,3)$ inversion transitions and the

\footnotetext{
11 The GBT is operated by the National Radio Astronomy Observatory, which is a facility of the National Science Foundation operated under cooperative agreement by Associated Universities, Inc.
}

$J_{\mathrm{K}_{+}, \mathrm{K}_{-}}=6_{1,6}-5_{2,3} 22 \mathrm{GHz} \mathrm{H}_{2} \mathrm{O}$ maser line with the $\mathrm{GBT}^{11}$ toward 1215 new BGPS sources. Dunham et al. (2011b) observed 631 BGPS sources within narrow ranges in Galactic longitude, while the new data presented in this work observed BGPS clumps that were detected in dense gas tracer $\mathrm{HCO}^{+} 3-2$ and had $T_{\mathrm{pk}}\left(\mathrm{HCO}^{+}\right)>0.3 \mathrm{~K}$ from Schlingman et al. (2011) and Shirley et al. (2013). All new observations were targeted with the center pixel of the $K$-band Focal Plane Array (KFPA) toward the $S_{1.1 \mathrm{~mm}}$ flux density peak in the BGPS v2.0 maps (Ginsburg et al. 2013). We measure $\mathrm{NH}_{3}$ properties using the slab model previously adopted in Dunham et al. (2011b). This model assumes a uniform-temperature, beam-filling slab of $\mathrm{NH}_{3}$ with column density $N\left(\mathrm{NH}_{3}\right)$, intrinsic velocity dispersion $\sigma_{\mathrm{v}}$, kinetic temperature $T_{\mathrm{K}}$, and line-of-sight velocity $v_{\mathrm{LSR}}$. The model assumes equal column densities of ortho- and para- $\mathrm{NH}_{3}$ and that the levels are populated in thermodynamic equilibrium. Given these assumptions, we use a nonlinear leastsquares regression to determine the optimal emission model incorporating the hyperfine structure and opacity of each component that matches the observed data (Rosolowsky et al. 2008). As in previous pointed spectroscopy studies, the $\mathrm{NH}_{3}$ fitting provides an excellent reproduction of the observed data, with the derived parameters representing emission-weighted averages of the physical conditions within the GBT beam. The observational setup, data reduction methods, and $\mathrm{NH}_{3}$ fitting techniques are described in detail in Dunham et al. (2010, 2011b).

Previous KFPA observations based on the BGPS v1.0 are updated to match the v2.0 based on the coincidence of the pointing within the BGPS label mask (discussed in Section 3.1 describing the catalog cross-matching method). Because of the changes in the BGPS catalog between data releases, 178 pointings are duplicates within the same v2.0 clumps and 245 pointings are not within a half-beam of a v2.0 clump. We use the observation closest to the peak flux density $S_{1.1 \mathrm{~mm}}$ position when there are multiple $\mathrm{NH}_{3}$ observations within the clump. Columns are included in Table 2 for the Bolocat v2.0 source and coordinate offset from the peak flux position. Table 2 also includes the observations from Dunham et al. (2011b) for consistency with the transition to BGPS v2.0. We find an overall detection rate in $\mathrm{NH}_{3}(1,1)$ at $75 \%$. For accurate estimates of the kinetic temperature we require a source to have $>3 \sigma$ detections in both the $(1,1)$ and $(2,2)$ transitions, resulting in 1663 clumps with suitable $T_{\mathrm{K}}$ fits. Table 2 lists the observed $\mathrm{NH}_{3}$ properties from the fits and $3 \sigma$ upper limits for nondetections. Unique $T_{\mathrm{K}}$ measurements are added for 48 BGPS clumps in $10^{\circ}<\ell<65^{\circ}$ from observations in Wienen et al. (2012).

\subsection{New $\mathrm{GBT}_{2} \mathrm{O}$ Maser Observations}

The $22 \mathrm{GHz} \mathrm{H}_{2} \mathrm{O}$ maser line is a well-known tracer of protostellar activity and was simultaneously observed in the fourth intermediate frequency (IF) alongside the $\mathrm{NH}_{3}(1,1)-(3$, 3) inversion transitions. A separate reduction in GBTIDL is used for the $\mathrm{H}_{2} \mathrm{O}$ maser data because of the complex structure in the spectra from multiple overlapping maser spots in the beam, strong baseline ripples, and a telluric feature at the topocentric zero velocity. The observations are dual-frequency switched with a $5 \mathrm{MHz}\left(69 \mathrm{~km} \mathrm{~s}^{-1}\right)$ throw. The telluric feature is pressure broadened by $\sim 2 \mathrm{MHz}\left(\sim 30 \mathrm{~km} \mathrm{~s}^{-1}\right)$ and adds significant structure to the baseline when frequency switched. We baseline fit the spectra with first- to third-order polynomials 
Table 1

Observed $\mathrm{H}_{2} \mathrm{O}$ Maser Properties

\begin{tabular}{|c|c|c|c|c|c|c|c|c|}
\hline $\begin{array}{l}\text { ID } \\
\text { Number }\end{array}$ & $\begin{array}{l}\text { GBT R.A. } \\
\text { (J2000) }\end{array}$ & $\begin{array}{l}\text { GBT decl. } \\
\text { (J2000) }\end{array}$ & $\begin{array}{c}\theta_{\text {offset }} \\
(\operatorname{arcsec})\end{array}$ & $\begin{array}{c}v_{\mathrm{LSR}}(\text { peak }) \\
\left(\mathrm{km} \mathrm{s}^{-1}\right)\end{array}$ & $\begin{array}{c}v_{\text {spread }} \\
\left(\mathrm{km} \mathrm{s}^{-1}\right)\end{array}$ & $\begin{array}{l}T_{\mathrm{mb}} \\
(\mathrm{K})\end{array}$ & $\begin{array}{c}W \\
\left(\mathrm{~K} \mathrm{~km} \mathrm{~s}^{-1}\right)\end{array}$ & $N_{\text {lines }}$ \\
\hline 2380 & 180749.6 & -200143.2 & 5.9 & 49.97 & 5.59 & $2.94(0.17)$ & $6.67(0.22)$ & 2 \\
\hline 2381 & 180841.6 & -200740.6 & 13.2 & 6.85 & 1.31 & $1.94(0.17)$ & $1.98(0.12)$ & 1 \\
\hline 2386 & 180848.1 & -200555.0 & 9.1 & 6.04 & 12.67 & $25.23(0.17)$ & $31.67(0.26)$ & 4 \\
\hline 2392 & 180923.1 & -200808.7 & 10.9 & 42.93 & 23.69 & $29.36(0.20)$ & $34.43(0.25)$ & 3 \\
\hline 2394 & 180902.2 & -200508.7 & 9.1 & 22.57 & 20.07 & $40.00(0.17)$ & $74.63(0.36)$ & 7 \\
\hline 2396 & 180900.6 & -200334.3 & 2.2 & 9.47 & 32.58 & $15.35(0.18)$ & $105.01(0.43)$ & 9 \\
\hline 2406 & 180921.5 & -200210.0 & 10.9 & 13.30 & 0.98 & $4.40(0.17)$ & $2.99(0.12)$ & 1 \\
\hline 2411 & 180845.1 & -195433.2 & 2.4 & 71.05 & 5.59 & $60.60(0.20)$ & $95.22(0.23)$ & 3 \\
\hline 2415 & 180901.7 & -194814.0 & 12.7 & 67.53 & 1.64 & $5.33(0.17)$ & $5.25(0.14)$ & 1 \\
\hline 2418 & 181022.5 & -195631.8 & 4.6 & 2.06 & 3.45 & $1.13(0.18)$ & $4.09(0.24)$ & 1 \\
\hline
\end{tabular}

Note. Uncertainties are given in parentheses.

(This table is available in its entirety in machine-readable form.)

to accommodate the complex baseline morphology. Spectra are required to have a $>5 \sigma$ detection in a single channel to qualify as detections. Table 3 lists the observed $\mathrm{H}_{2} \mathrm{O}$ maser properties and $3 \sigma$ upper limits for nondetections. These values include the R.A. and decl. of the GBT pointing, offset from the BGPS peak position $\theta_{\text {offset }}$, velocity of the peak component $v_{\text {LSR }}$, FWZI spread between the left- and rightmost velocity components $v_{\text {spread, }}$, main-beam peak intensity $T_{\mathrm{mb}}$, main-beam integrated intensity $W$, and number of unblended velocity components $N_{\text {lines }}$. We observe 472 detections, 343 of which are unique associations not contained in the published surveys by the Mopra and Arcetri observatories discussed in Section 3.3.1. No significant deviation was seen between the center velocity defined by the first moment and that of the strongest peak. The number of unblended line peaks is identified through visual inspection, but because the maser components can be heavily blended toward strong sources, this number represents a lower limit to the number of maser components toward a BGPS source. For extreme $\mathrm{H}_{2} \mathrm{O}$ maser emission, the weaker telluric feature is covered and adds a $\sim 0.5 \mathrm{~K} \mathrm{~km} \mathrm{~s}^{-1}$ uncertainty to the calculated $\mathrm{H}_{2} \mathrm{O}$ maser integrated intensity. This overlap introduces a $<1 \%$ uncertainty in the $>100 \mathrm{~K} \mathrm{~km} \mathrm{~s}^{-1} \mathrm{H}_{2} \mathrm{O}$ maser spectra where this occurs.

\section{DEVELOPING A STAR FORMATION INDICATOR CATALOG}

Recently completed blind Galactic plane surveys with wavelength coverage from the near-infrared to radio provide a wide range of indicators of star formation activity. We develop an evolutionary catalog for clumps in the BGPS based on the indicators of star formation that can be associated with the clumps. These evolutionary indicators are then grouped into the categories so that their observed and physical properties can be compared. From this catalog, we identify a subsample of SCCs that lack indicators of protostellar activity. Below we describe the surveys and data sets used to define the flags of star formation indicators. The published surveys include the Red MSX Survey (RMS; Lumsden et al. 2002), Glimpse Red Source Catalog (Robitaille et al. 2008), Hi-GAL $70 \mu \mathrm{m}$ images (Molinari et al. 2010), $\mathrm{H}_{2} \mathrm{O}$ Southern Galactic Plane Survey (Walsh et al. 2011), Methanol Multibeam Maser Survey (Breen et al. 2015), and Coordinated Radio and Infrared Sky Survey for High-mass Star Formation (CORNISH; Hoare et al. 2012), in addition to the GBT $\mathrm{H}_{2} \mathrm{O}$ maser and $\mathrm{NH}_{3}$ observations presented in this paper. We aim for the most uniform coverage in the surveys to accurately compare star formation indicator populations. The common overlap region among the surveys described below is in the range $10^{\circ}<\ell<65^{\circ}$.

\subsection{Catalog Cross-matching}

All published catalogs are cross-matched to the BGPS v2.0 catalog based on the line-of-sight coincidence on the sky. The Bolocat modified seeded watershed algorithm extracts the clump catalog from the Bolocam data and defines the spatial extent of each clump (Rosolowsky et al. 2010). In this context the "label map" or "label mask" refers to the pixels in the Bolocam $1.1 \mathrm{~mm}$ flux image associated with a clump by the watershed. For a source from another catalog to be matched with a clump we require that it must be contained within the clump label mask. In the common overlap region between $10^{\circ}<\ell<65^{\circ}$ there are 4683 BGPS clumps and 2203 clumps with either $\mathrm{HCO}^{+}$or $\mathrm{NH}_{3}$ dense gas detections. Table 1 lists the observed $1.1 \mathrm{~mm}$ properties for the 4683 BGPS clumps within the common overlap region. Table 4 shows the detection statistics for the cross-matched catalogs described in Sections 3.2.1-3.4 to the BGPS. The table lists the number of sources in the overlap region, the number of sources matched with BGPS clumps, and the number of BGPS clumps containing at least one matched source.

Without accurate independent distances for both a BGPS clump and a catalog source for a star formation tracer, we must rely on spatial coincidence on the sky to cross-match sources. To assess the accuracy of the cross-matched associations, Dunham et al. (2011a) estimate the rate of chance alignments statistically. Dunham et al. (2011a) compare the fraction of cross-matched sources in a given catalog to the average fraction of sources cross-matched when the coordinates have been randomized on area much greater than a single BGPS clump's environment (see Table 1). Randomizing the coordinates of the catalog preserves the large-scale projected spatial distribution of sources throughout the Galaxy. If the sources in a catalog are quasi-uniformly distributed on the sky, this randomized overlap fraction would simply be the area of the sky covered by BGPS clumps divided by the survey coverage. However, because of Galactic structure and line-of-sight projection effects, the distribution of most catalog sources (e.g., red GLIMPSE sources) will not be uniform, and this will be reflected in a higher count of randomized cross-matches toward, e.g., the 
Observed $\mathrm{NH}_{3}$ Properties

\begin{tabular}{|c|c|c|c|c|c|c|c|c|c|c|c|}
\hline $\begin{array}{l}\text { ID } \\
\text { Number }^{\mathrm{a}}\end{array}$ & $\begin{array}{c}\text { GBT R.A. } \\
\text { (J2000) }\end{array}$ & $\begin{array}{l}\text { GBT decl. } \\
\text { (J2000) }\end{array}$ & $\begin{array}{c}\theta_{\text {offset }} \\
(\operatorname{arcsec})\end{array}$ & $\begin{array}{c}v_{\mathrm{LSR}} \\
\left(\mathrm{km} \mathrm{s}^{-1}\right)\end{array}$ & $\begin{array}{c}\sigma_{\mathrm{v}} \\
\left(\mathrm{km} \mathrm{s}^{-1}\right)\end{array}$ & $\begin{array}{c}T_{\mathrm{mb}}(1,1) \\
(\mathrm{K})\end{array}$ & $\begin{array}{c}W(1,1) \\
\left(\mathrm{K} \mathrm{km} \mathrm{s}^{-1}\right)\end{array}$ & $\begin{array}{c}T_{\mathrm{mb}}(2,2)^{\mathrm{b}} \\
(\mathrm{K})\end{array}$ & $\begin{array}{c}W(2,2)^{\mathrm{b}} \\
\left(\mathrm{K} \mathrm{km} \mathrm{s}^{-1}\right)\end{array}$ & $\begin{array}{c}T_{\mathrm{mb}}(3,3)^{\mathrm{b}} \\
(\mathrm{K})\end{array}$ & $\begin{array}{c}W(3,3)^{\mathrm{b}} \\
\left(\mathrm{K} \mathrm{km} \mathrm{s}^{-1}\right)\end{array}$ \\
\hline 2351 & 180728.3 & $\begin{array}{llll}-20 & 14 & 10.3\end{array}$ & 12.9 & $30.1300(0.1600)$ & $0.6900(0.1600)$ & $0.73(0.20)$ & $1.57(0.58)$ & $0.58(0.20)$ & $-0.06(0.58)$ & $0.67(0.22)$ & $0.83(0.58)$ \\
\hline 2356 & 180925.6 & -202543.6 & 11.0 & $11.7570(0.0170)$ & $0.8700(0.0160)$ & $3.82(0.22)$ & $32.32(0.64)$ & $1.37(0.20)$ & $3.07(0.63)$ & $0.88(0.22)$ & $0.21(0.64)$ \\
\hline 2358 & 180838.8 & -201858.9 & 6.1 & $28.2010(0.0170)$ & $0.8770(0.0160)$ & $3.62(0.21)$ & $27.42(0.62)$ & $1.29(0.19)$ & $3.35(0.62)$ & $0.88(0.23)$ & $0.20(0.69)$ \\
\hline 2360 & 180932.3 & -202407.7 & 11.5 & $11.7862(0.0098)$ & $0.8173(0.0093)$ & $6.03(0.21)$ & $42.04(0.67)$ & $2.39(0.20)$ & $4.62(0.69)$ & $1.02(0.21)$ & $0.05(0.74)$ \\
\hline 2361 & 180801.4 & $\begin{array}{lll}-20 & 12 & 19.1\end{array}$ & 10.8 & $41.8440(0.0160)$ & $1.0290(0.0140)$ & $4.04(0.21)$ & $34.86(0.64)$ & $2.04(0.20)$ & $5.65(0.68)$ & $0.92(0.22)$ & $-0.71(0.74)$ \\
\hline 2367 & $\begin{array}{lll}18 & 09 & 27.0\end{array}$ & $\begin{array}{llll}20 & 19 & 11.2\end{array}$ & 3.5 & $14.1900(0.0220)$ & $2.2420(0.0210)$ & $4.92(0.25)$ & $68.37(0.90)$ & $3.81(0.22)$ & $20.81(0.86)$ & $4.55(0.33)$ & $30.31(1.28)$ \\
\hline 2372 & 180936.9 & $\begin{array}{llll}-20 & 18 & 41.8\end{array}$ & 9.3 & $10.7959(0.0046)$ & $0.8328(0.0041)$ & $11.89(0.23)$ & $90.16(0.69)$ & $6.88(0.21)$ & $25.18(0.69)$ & $3.03(0.23)$ & $10.36(0.76)$ \\
\hline 2376 & $\begin{array}{lll}18 & 09 & 05.4\end{array}$ & $\begin{array}{lll}20 & 13 & 39.7\end{array}$ & 2.0 & $13.2780(0.0240)$ & $0.8210(0.0240)$ & $2.59(0.21)$ & $16.26(0.61)$ & $1.04(0.20)$ & $0.18(0.62)$ & $0.84(0.24)$ & $-0.43(0.70)$ \\
\hline 2378 & $\begin{array}{lll}18 & 09 & 24.8\end{array}$ & -201537.6 & 2.1 & $10.9600(0.0055)$ & $1.3419(0.0044)$ & $12.33(0.23)$ & $168.72(0.74)$ & $8.47(0.22)$ & $52.84(0.76)$ & $5.10(0.24)$ & $28.28(0.86)$ \\
\hline 2379 & 180900.3 & -201137.5 & 6.2 & $11.7906(0.0033)$ & $0.7766(0.0031)$ & $16.59(0.23)$ & $114.18(0.70)$ & $9.21(0.21)$ & $33.00(0.70)$ & $4.87(0.26)$ & $17.92(0.83)$ \\
\hline
\end{tabular}

Notes. Uncertainties are given in parentheses.

${ }^{\mathrm{a}} \mathrm{B}, \mathrm{C}, \mathrm{D}$, and $\mathrm{E}$ denote multiple ammonia pointings that fall within a single $1.1 \mathrm{~mm}$ source.

${ }^{\mathrm{b}}$ Upper limits are $T_{\mathrm{mb}}<4 \sigma$ and $W<5 \sigma \Delta v \sqrt{N}$, where $\sigma$ is the rms noise, $\Delta v$ is the width of a single channel in velocity, and $N$ is the number of pixels over which the average rms was calculated.

(This table is available in its entirety in machine-readable form.) 
Table 3

Observed $1.1 \mathrm{~mm}$ Properties

\begin{tabular}{|c|c|c|c|c|c|c|c|c|c|}
\hline $\begin{array}{l}\text { ID } \\
\text { Number }\end{array}$ & Source & $\begin{array}{c}\text { Peak R.A. } \\
(\mathrm{J} 2000)\end{array}$ & $\begin{array}{c}\text { Peak decl. } \\
(\text { J2000) }\end{array}$ & $\begin{array}{c}\Omega^{\mathrm{FWHM}} \\
\left(\operatorname{arcmin}^{2}\right)\end{array}$ & $\begin{array}{c}\Omega^{\text {Total }} \\
\left(\operatorname{arcmin}^{2}\right)\end{array}$ & $\begin{array}{l}\theta_{\mathrm{eq}}^{\mathrm{FWHM}} \\
(\operatorname{arcsec})\end{array}$ & $\begin{array}{c}\theta_{\mathrm{eq}}^{\text {Total }} \\
(\operatorname{arcsec})\end{array}$ & $\begin{array}{c}S_{1.1}^{\mathrm{FWHM}} \\
(\mathrm{mJy})\end{array}$ & $\begin{array}{l}S_{1.1}^{\text {Total }} \\
(\mathrm{mJy})\end{array}$ \\
\hline 2351 & BGPSv2_G010.013 + 00.082 & 180729.0 & -201418.0 & 1.613 & 2.534 & 39.7 & 51.3 & $760(160)$ & $1000(200)$ \\
\hline 2352 & BGPSv2_G010.027-00.354 & 180908.3 & -202615.8 & 1.598 & 3.456 & 39.5 & 60.7 & $1420(190)$ & $2110(290)$ \\
\hline 2353 & BGPSv2_G010.051-00.208 & 180838.6 & -202045.5 & 0.734 & 0.994 & 23.9 & 29.4 & $420(120)$ & $490(140)$ \\
\hline 2354 & BGPSv2_G010.059-00.210 & 180840.0 & -202023.8 & 0.965 & 1.037 & 28.9 & 30.3 & $250(110)$ & $290(120)$ \\
\hline 2355 & BGPSv2_G010.061-00.174 & 180832.2 & -201914.7 & 1.397 & 1.930 & 36.4 & 44.0 & $780(160)$ & $930(180)$ \\
\hline 2356 & BGPSv2_G010.069-00.406 & 180925.2 & -202534.2 & 1.210 & 5.328 & 33.4 & 76.4 & $1050(160)$ & $2750(350)$ \\
\hline 2357 & BGPSv2_G010.079-00.414 & 180928.2 & -202516.6 & 0.778 & 1.080 & 24.9 & 31.1 & $570(120)$ & $680(140)$ \\
\hline 2358 & BGPSv2_G010.079-00.194 & 180838.9 & -201852.9 & 1.670 & 3.096 & 40.5 & 57.2 & $1510(190)$ & $2010(250)$ \\
\hline 2359 & BGPSv2_G010.083-00.434 & 180933.2 & -202538.8 & 0.547 & 0.763 & 18.8 & 24.5 & $310(100)$ & $390(120)$ \\
\hline 2360 & BGPSv2_G010.105-00.416 & 180931.9 & -202358.2 & 0.936 & 2.117 & 28.3 & 46.4 & $850(140)$ & $1320(210)$ \\
\hline
\end{tabular}

Note. Uncertainties are given in parentheses.

(This table is available in its entirety in machine-readable form.)

inner Galaxy. The randomized cross-matching yields typically between $7 \%$ and $9 \%$ of catalog sources being associated with BGPS clumps. In this work we use the BGPS v2.0, whereas Dunham et al. (2011a) used the BGPS v1.0. We find similar results for the RMS, EGO, and Robitaille et al. (2008, hereafter R08) sources matched to clumps (Section 3.2) and the number of clumps with associated sources within a few percent of the total fraction of matched sources. Catalogs that are new additions in this work such as the Methanol Multibeam Survey (MMB) and CORNISH also have high association rates, $>80 \%$, so we do not suspect that the sources in these catalogs are highly contaminated by chance alignments. Furthermore, other data sets in this work such as the Hi-GAL visual inspection and the GBT $\mathrm{H}_{2} \mathrm{O}$ observations are targeted, and the coordinates cannot be randomized.

\subsection{Infrared Surveys \\ 3.2.1. Red MSX Survey}

The RMS (Lumsden et al. 2002, 2013) with observations at $8,12,14$, and $21 \mu \mathrm{m}$ defines a sample of massive YSOs complete above $L_{\text {bol }}>10^{4} L_{\odot}$ and $d_{\odot}<10 \mathrm{kpc}$, approximately a B0-type main-sequence star. The RMS catalog region has complete overlap with the BGPS for $\ell>10^{\circ}$, where confusion does not dominate the $M S X$ data. Extensive followup observations of RMS sources yield detailed classifications: protoplanetary nebula, planetary nebula, evolved star, H II region, YSO, OH/IR star, young/old star, $\mathrm{H}$ II/YSO, carbon star, and a miscellaneous "other." We use the RMS classifications associated with YSOs (YSO, H II/YSO, young/old star) to classify BGPS sources in the mid-IR category. We do not associate BGPS sources with star formation activity for the other categories because contamination between the RMS classifications is low. This is further evidenced by the minimal overlap in Table 4. The RMS sources provide a well-vetted sample of massive YSOs, but for spectral types later than B0, or YSOs that are at distances $>10 \mathrm{kpc}$, the survey is incomplete. Recent Galactic plane surveys by Spitzer and Herschel offer sensitive data sets through the mid- and far-IR to probe the star formation activity in BGPS clumps and complement the RMS data.

\subsubsection{GLIMPSE Red Source Catalog}

The GLIMPSE survey is a Spitzer/IRAC Legacy survey of the Galactic midplane, at 3.6, 4.5, 5.8, and $8.0 \mu \mathrm{m}$ (Benjamin et al. 2003; Churchwell et al. 2009). The GLIMPSE I and II fields provide complete overlap coverage with the BGPS for $10^{\circ}<\ell<65^{\circ}$. R08 use the GLIMPSE catalog to select a total number of 18,949 intrinsically red $([4.5]-[8.0] \geqslant 1)$ sources that also meet photometric quality criteria. MIPS $24 \mu \mathrm{m}$ magnitudes are also calculated at the same positions for $86.9 \%$ of the sources from the MIPSGAL survey (Carey et al. 2009). Because GLIMPSE has better spatial resolution and sensitivity than the $M S X$ survey, lower-luminosity YSOs can be detected. R08 estimate that a $100 L_{\odot}$ Stage I YSO should be detected at distances between 0.2 and $10 \mathrm{kpc}$ and a $10^{4} L_{\odot}$ YSO between 2 and $30 \mathrm{kpc}$. Intrinsically red sources are further classified by color-magnitude and color-color selection criteria (see Equations (7)-(9) Robitaille et al. 2008) into candidate YSOs, "standard" $\mathrm{C}$ - and O-rich asymptotic giant branch (AGB) stars (sAGB), and "extreme" AGB stars (xAGB). The color criteria select sources with spectral index $\alpha \geqslant-1.2$, which, following the "Class" system of Greene et al. (1994), selects all Class I and a significant number of Class II YSOs. Contamination from planetary nebula and background galaxies is expected to be between $2 \%$ and $3 \%$. However, because AGB and YSO sources have similar mid-IR colors, the YSO/AGB discrimination is uncertain based on 4.5, 8.0 , and $24 \mu \mathrm{m}$ photometry alone, with up to $50 \%$ contamination between the two categories. Dunham et al. (2011b) estimate a $\sim 40 \%$ contamination fraction of YSOs in the R08 candidate AGB sources. We shall account for this contamination in the MC sampling in Section 5. The number of R08 candidate YSO or AGB sources matched to BGPS clumps is listed in Table 4.

An additional class of sources called extended green objects (EGOs) have been identified from the Spitzer GLIMPSE data (Cyganowski et al. 2008; Chen et al. 2013). EGOs are sources of extended $4.5 \mu \mathrm{m}$ emission from shocked $\mathrm{H}_{2}$ driven by strong outflows from massive star formation. A total of 72 BGPS clumps match EGOs, and all except one clump (G044.00800.026) contain RMS YSOs, R08 YSOs, or $70 \mu \mathrm{m}$ compact sources (see Section 3.2.4). 
Table 4

Catalog Matching Statistics

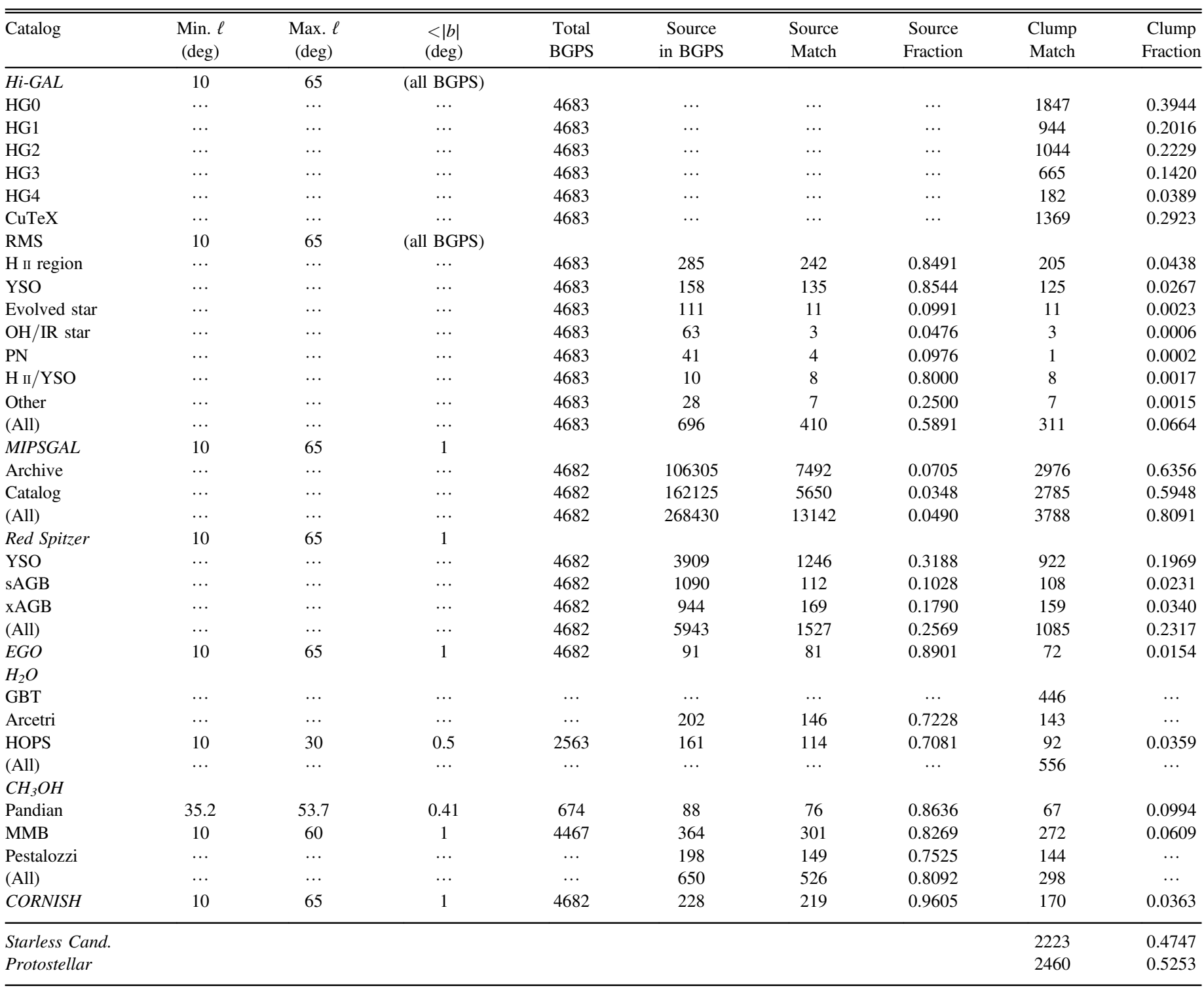

Note. Columns list the (1) catalog matched to the BGPS, (2) minimum Galactic longitude of the survey if applicable, (3) maximum Galactic longitude, (4) range in Galactic latitude, (5) total number of BGPS clumps in the catalog survey region, (6) number of catalog sources in the region with overlapping BGPS coverage, (7) number of catalog sources associated with BGPS clumps, (8) fraction of catalog sources matched to BGPS clumps, (9) number of unique associated BGPS clumps, and (10) fraction of BGPS clumps with associations.

\subsubsection{MIPSGAL $24 \mu \mathrm{m}$}

The Version 3.0 data products from the Spitzer MIPSGAL survey cover $-69^{\circ}<\ell<68^{\circ}$ and $|b|<1^{\circ}$ at $24 \mu \mathrm{m}$ (Carey et al. 2009). The MIPS $24 \mu \mathrm{m}$ band is sensitive to the hot dust found around YSOs and $\mathrm{H}$ II regions, but also to envelopes of evolved stars. Gutermuth \& Heyer (2015) have created a comprehensive point-source catalog from the MIPSGAL $24 \mu \mathrm{m}$ maps. Two catalogs are presented: a high-quality catalog and more complete archive. Combined, the catalogs total 268,430 point sources in the fields of the BGPS overlap region, with $4.9 \%$ of MIPSGAL sources associated with clumps and $80.9 \%$ of clumps associated with MIPSGAL point sources. While some subset of MIPSGAL sources do represent observational indicators of embedded protostellar activity, this low match fraction of $4.9 \%$ is consistent with the $6.8 \% \pm 2.2 \%$ randomized match fraction calculated in Dunham et al. (2011b) for RMS evolved stars (i.e., sources with no physical association). Figure 2 illustrates this poor correspondence to BGPS clumps. Two maps at 70 and $24 \mu \mathrm{m}$ are shown of the same field centered on a single, isolated clump. Whereas the $24 \mu \mathrm{m}$ image shows identified point sources from both the catalog and archive, only a few point sources have direct association with the clump $1.1 \mathrm{~mm}$ emission. In contrast, the $70 \mu$ mimage uniquely associates a single compact source with the clump. The $70 \mu \mathrm{m}$ dust continuum emission is more sensitive to the deeply embedded star formation activity that is better positionally correlated to BGPS clumps. We conclude that line-of-sight association of $24 \mu \mathrm{m}$ point sources from the MIPSGAL catalog are not a sufficient sole indicator of star formation activity because of the high contamination from evolved stars. Thus, while the MIPSGAL flags are not used in 

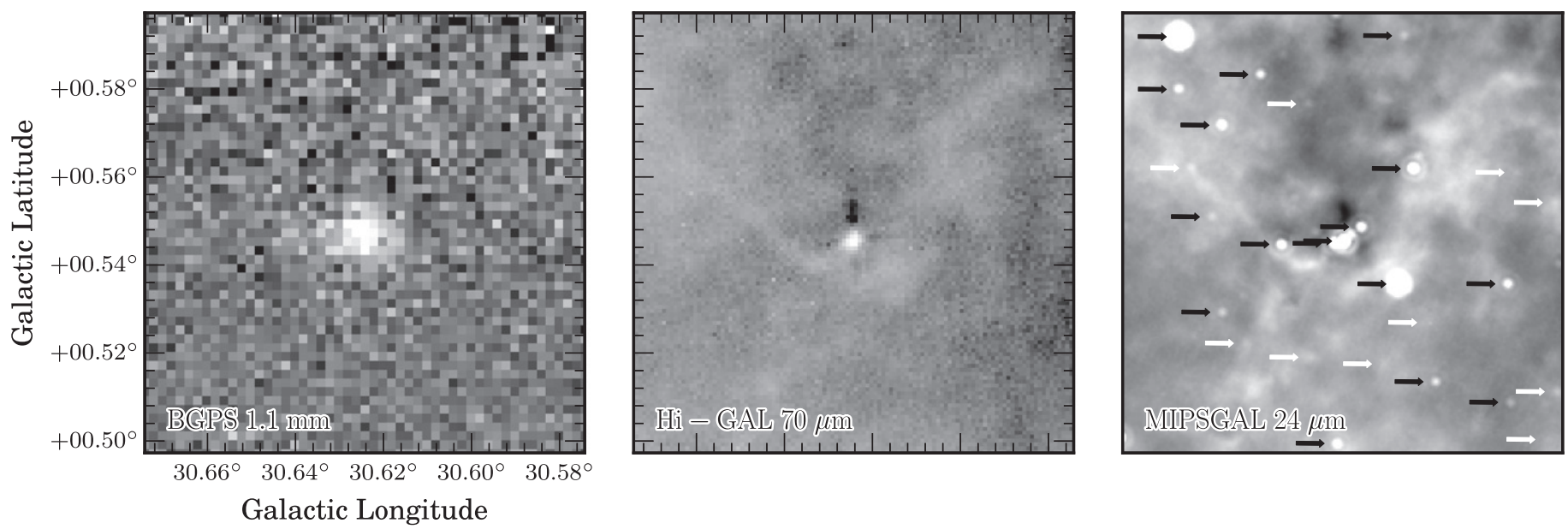

Galactic Longitude

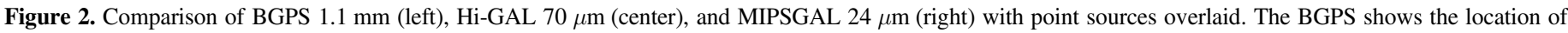

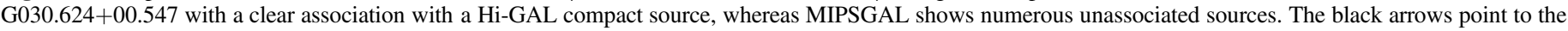
MIPSGAL high-quality catalog, and the white arrows point to the more complete archive (Gutermuth \& Heyer 2015).
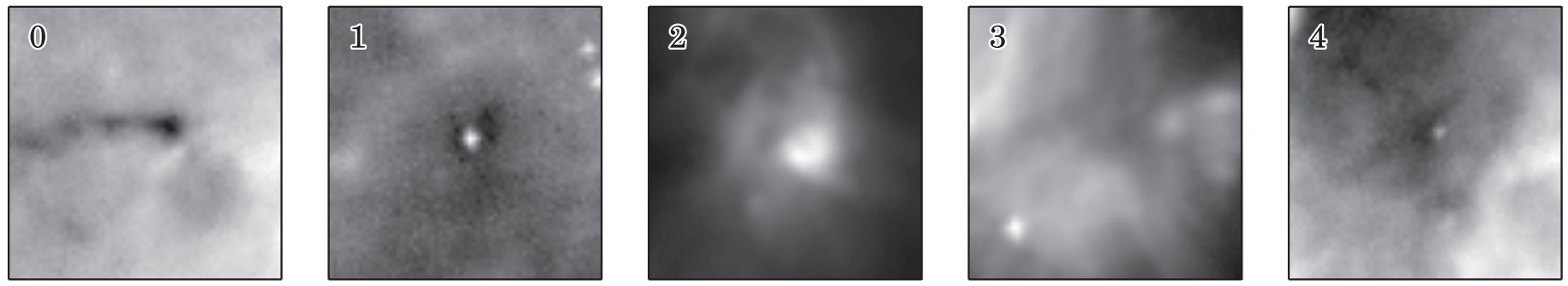

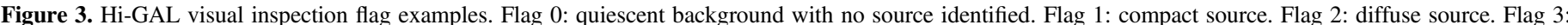
bright or complex background with no source identified. Flag 4: faint or low confidence source. The cutouts are $5^{\prime} \times 5^{\prime}$.

any calculations of physical properties, for completeness we present the flags in Table 4 (see Section 3.5). These contamination issues also strongly affect the lower-resolution WISE $22 \mu \mathrm{m}$ observations. To further refine our set of star formation indicators, rather than pursue color- and qualityselection criteria to identify a less contaminated subsample from the MIPSGAL catalog, we associate clumps with $70 \mu \mathrm{m}$ compact sources.

\subsubsection{Herschel Hi-GAL $70 \mu \mathrm{m}$}

The Hi-GAL is a far-IR survey of the Galaxy with the 70 and $170 \mu \mathrm{m}$ PACS bands and the 250, 350, and $500 \mu \mathrm{m}$ SPIRE bands (Molinari et al. 2010). The Hi-GAL survey completely covers the BGPS fields that we discuss. Previous survey data sets in the $100 \mu \mathrm{m}$ regime from IRAS and Akari are not suitable for detecting faint point sources owing to their poor angular resolution $\left(>1^{\prime}\right)$. The Herschel Hi-GAL 10 "' 2 resolution (Traficante et al. 2011) at $70 \mu \mathrm{m}$ is essential to disentangling the far-IR emission reprocessed by dust around embedded YSOs and their often active environment. Most important, however, is the less severe contamination in the Hi-GAL $70 \mu \mathrm{m}$ images from evolved stars compared to the MIPS $24 \mu \mathrm{m}$ or WISE $22 \mu \mathrm{m}$ images. Whereas $>80 \%$ clumps have at least one associated MIPSGAL source, only $46 \%$ of clumps have an associated $70 \mu \mathrm{m}$ compact source from visual identification with clearly correlated positions to the $1.1 \mathrm{~mm}$ maps.

Owing to complicated background structure, identifying compact sources algorithmically is difficult. The CuTeX algorithm (Molinari et al. 2011) was developed specifically for identifying sources in highly spatially variable backgrounds such as those found in the Hi-GAL images. Careful visual inspection is necessary because of false negatives, where CuTeX misses faint sources, and false positives, where CuTeX misidentifies sources in regions of strong emission. Cuts on CuTeX fit parameters were trained on publicly available data in the $\ell=30^{\circ}$ field (Bally et al. 2010) and then applied to all cutout images to compare with the results of the visual inspection classifications. The comparisons show that $77.7 \%$ of the classifications agree and $22.3 \%$ disagree; $81 \%$ of the disagreements originate from sources flagged with fainter $70 \mu \mathrm{m}$ sources from the visual inspection but missed by cuTeX.

We visually inspect $5^{\prime} \times 5^{\prime}$ cutouts of the reduced Hi-GAL $70 \mu \mathrm{m}$ band images (Molinari et al. 2011) for comparison against the BGPS data. For consistency with the catalogmatching scheme used for the other surveys in this study, we classify clumps as containing a Hi-GAL $70 \mu \mathrm{m}$ source if it is within the Bolocat label mask. Visual inspection was carried out on the $70 \mu \mathrm{m}$ images alone, without simultaneous comparison to GLIMPSE or MIPSGAL images to prevent systematic bias in the classifications. We assign BGPS clumps one of five flags in the visual inspection process. Multiple flags are necessary to qualify the image background level and source characteristics. Flags 1, 2, and 4 represent clumps identified with a compact source within the label mask, while flags 0 and 3 lack a clearly identifiable source. Representative $70 \mu \mathrm{m}$ cutouts of these flags are shown in Figure 3. Flag 1's show a high confidence compact source on the scale of the Herschel 10". 2 point-spread function (PSF). Flag 2's are more diffuse than the PSF but identifiable as compact sources 
owing to strong, localized emission above the background level. These are common for extremely bright $70 \mu \mathrm{m}$ sources with $\geqslant 80 \%$ of uniquely high-mass indicators, RMS sources, $\mathrm{CH}_{3} \mathrm{OH}$ masers, and $\mathrm{UCH}_{\mathrm{II}}$ regions, assigned flag 2's. We identify 944 flag 1 and 1044 flag 2 clumps, or $42.5 \%$ of clumps. Flag 4's show a weak, PSF-like source whose classification is lower confidence. Adding the 182 flag 4's increases the total to $46.3 \%$ of clumps classified with a Hi-GAL source. The brightness and complexity of the $70 \mu \mathrm{m}$ background can make the visual source identification difficult. Because of this, we assign 1847 flag 0's to clumps with quiescent, smooth, and low-level backgrounds and 655 flag 3's to clumps with bright and more complex backgrounds. The far-IR background of the Milky Way changes locally and as a function of Galactic longitude (generally $10^{2}-$ $10^{3} \mathrm{MJy} \mathrm{sr}^{-1}$ ), and thus the flux threshold for source detection changes as well.

The high rate of overlap between $70 \mu \mathrm{m}$ sources and more extreme indicators, along with the large number of unique $70 \mu \mathrm{m}$ associations, suggests that the Hi-GAL data are broadly probing a lower YSO bolometric luminosity, $L_{\text {bol }}$, than the other indicators. To assess the low-luminosity limit of the associations, we compute $L_{\mathrm{bol}}$ completeness functions for a representative sample of background levels given the sample of DPDFs in the Distance Catalog. The $70 \mu \mathrm{m}$ background level and complexity are the limiting factors in the identification of compact sources. A detailed analysis of the background level distribution for the full Hi-GAL $70 \mu$ m data set with the identification threshold quantified through fake source injection is beyond the scope of this paper. Instead, we bracket the completeness function with a sample of 30 clumps associated with the least confident $70 \mu \mathrm{m}$ identifications (classified as HiGAL flag 4), evenly split between low background levels of 500-1000 $\mathrm{MJy} \mathrm{sr}^{-1}$ and high background levels of 2000-3000 $\mathrm{MJy} \mathrm{sr}^{-1}$. Values are calculated from the cutouts described above. Aperture photometry of the $70 \mu \mathrm{m}$ sources in these selected regions yields sources identified with flux densities $\lesssim 0.3$ Jy for the low backgrounds and $\lesssim 1$ Jy for the high backgrounds. Using these two detection thresholds, we compare to fluxes derived from the 20,000 axisymmetric YSO radiative transfer models computed in Robitaille et al. (2006, 2007). We use the PACS $70 \mu$ m fluxes from the largest physical aperture, $100,000 \mathrm{au}$, which corresponds to the $20^{\prime \prime}$ diameter aperture at the median clump distance of $5 \mathrm{kpc}$ (see Section 5.1.2). We then draw from MC simulations for each clump DPDF (described in Section 5.1) to scale the Robitaille et al. (2006) PACS $70 \mu \mathrm{m}$ model flux and apply the detection threshold cut. Figure 4 shows the completeness function for all clumps with well-constrained distances, and the cyan line shows the same but for the subset of clumps between $28^{\circ}<\ell<31^{\circ}$ for which publicly available Hi-GAL data exist (Bally et al. 2010). The dotted lines show the $50 \%$ and $90 \%$ limits. We find that in the low-background environments the $70 \mu \mathrm{m}$ visual flags are $50 \%$ complete to YSOs with $L_{\text {bol }}>10 L_{\odot}$ and $90 \%$ complete to YSOs with $L_{\text {bol }}>50 L_{\odot}$. For the high-background environments, we find the $50 \%$ completeness limit to YSOs at $L_{\text {bol }}>45 L_{\odot}$ and $90 \%$ complete to YSOs with $L_{\mathrm{bol}}>140 L_{\odot}$. To put these luminosities in perspective, a $\sim 1 M_{\odot}$ and $\sim 5 R_{\odot}$ YSO accreting at $\sim 10^{-5} M_{\odot} \mathrm{yr}^{-1}$ corresponds to an accretion luminosity of $\sim 30 L_{\odot}$ (N.B. episodic accretion rates result in large variations in the accretion luminosity at a given epoch; see Dunham

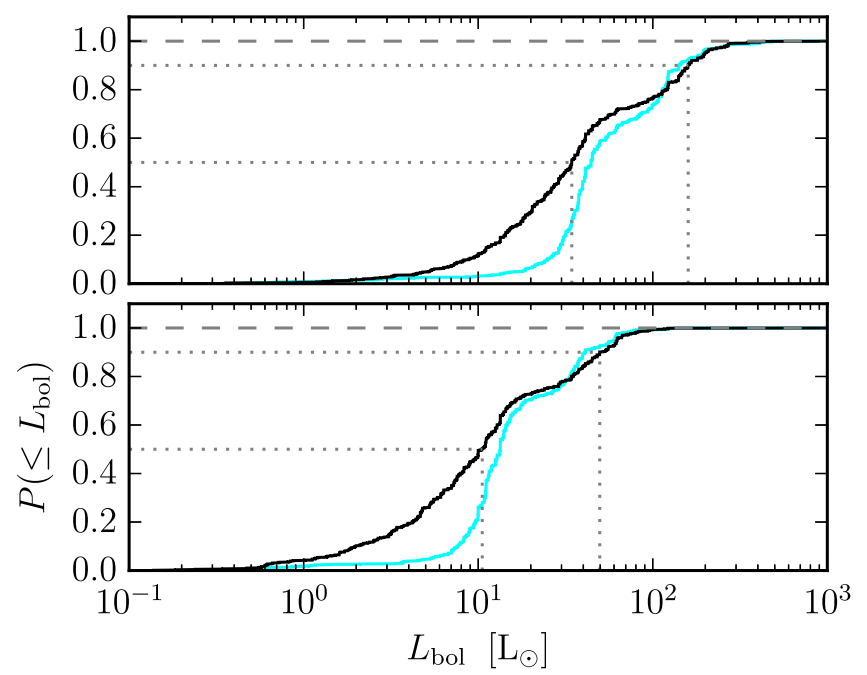

Figure 4. Completeness function for the bolometric luminosity, $L_{\mathrm{bol}}$, of HiGAL $70 \mu \mathrm{m}$ sources associated with BGPS clumps in the Distance Catalog (black line). Models from Robitaille et al. (2006) are used to compute $L_{\mathrm{bol}}$. Top: completeness in high-background (2000-3000 $\mathrm{MJy} \mathrm{sr}^{-1}$ ) regions calculated with source detection threshold of $1 \mathrm{Jy}$. Bottom: completeness in lowbackground (500-1000 $\mathrm{MJy} \mathrm{sr}^{-1}$ ) regions calculated with source detection threshold of $0.3 \mathrm{Jy}$. Dotted lines show the $50 \%$ and $90 \%$ completeness limits, and the cyan line shows the completeness function using only DPDFs between $\ell=28^{\circ}$ and $31^{\circ}$.

et al. 2014). While the true sample completeness limits likely lie between these two values, it should be kept in mind that these limits are below the $L_{\mathrm{bol}}$ of the other star formation indicators by several orders of magnitude.

Among the positive Hi-GAL classifications, 1027/2170 $(47.3 \%)$ have one or more additional star formation indicator and $1143 / 2170(52.7 \%)$ are Hi-GAL $70 \mu$ m unique, representative of a sample of deeply embedded and early protostellar candidates. In the following analysis, this sample of clumps uniquely identified with star formation activity from $70 \mu$ m compact sources is treated separately as its own category. It should be noted that the $70 \mu \mathrm{m}$ unique category sources may have an MIPS $24 \mu \mathrm{m}$ counterpart but do not contain any other star formation indicator (unique is defined in terms of our set of star formation indicators).

\subsection{Masers}

\subsection{1. $\mathrm{H}_{2} \mathrm{O}$ Masers}

We complement the mid- and far-IR star formation indicators with radio observations of masers. The outflows driven by young protostars shock dense regions of the ISM and collisionally pump $\mathrm{H}_{2} \mathrm{O}$ that creates a population inversion that drives the maser emission. The typical physical conditions for a masing region are a spatial density $n \sim 10^{7}-10^{9} \mathrm{~cm}^{-3}$, kinetic temperature $T_{\mathrm{K}} \sim 400 \mathrm{~K}$, spatial extent of $d \sim 0.66 \mathrm{au}$, and an aspect ratio of $\sim 50: 1$ (Hollenbach et al. 2013). $\mathrm{H}_{2} \mathrm{O}$ masers are time variable on scales of months to years. The beaming effect of $\mathrm{H}_{2} \mathrm{O}$ masers makes them a sensitive probe at large distances, but because of the necessary narrow geometrical alignment, the nondetection of an $\mathrm{H}_{2} \mathrm{O}$ maser does not imply a lack of star formation activity. In addition to YSO molecular outflows, $\mathrm{H}_{2} \mathrm{O}$ masers are also observed in the shells around AGB stars (Imai et al. 2002). From high-resolution follow-up of the $\mathrm{H}_{2} \mathrm{O}$ Southern Galactic Plane Survey (HOPS; Walsh et al. 2011) and association with catalogs of star formation indicators, Walsh 


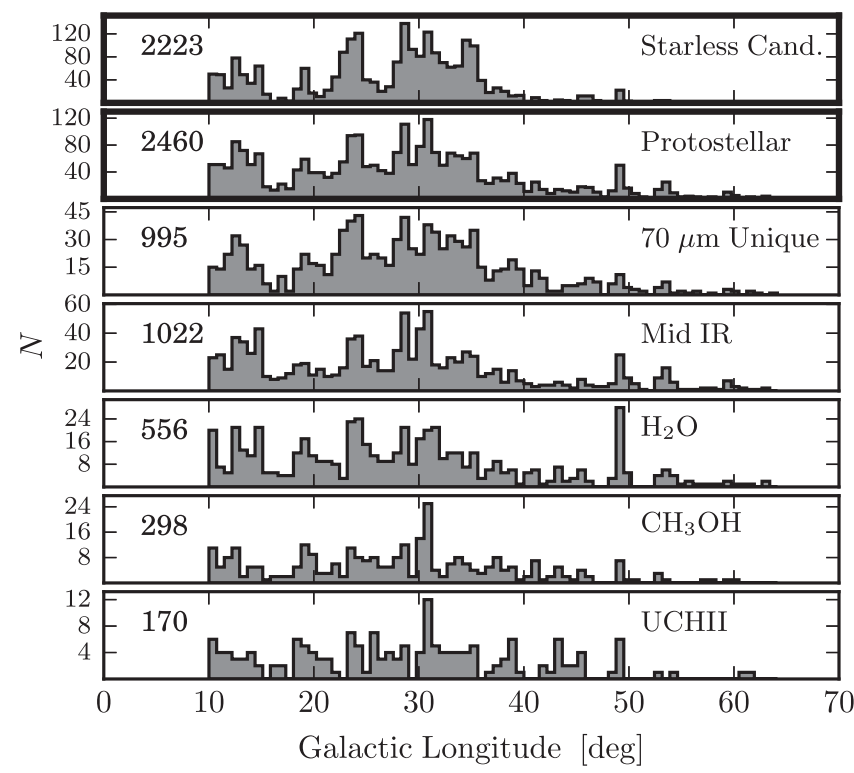

Figure 5. Observed distributions in Galactic longitude for different star formation indicators. The top two panels show the distributions for starless clump candidates and protostar-containing clumps for all BGPS clumps in the overlap region. The bottom five panels are ordered by star formation indicator, ranked by the approximate luminosity of a single protostar that produces the indicator. The number of BGPS clumps in each subset is shown in the upper left. Note that the $y$-axis scaling is different for each panel.

et al. (2014) find that $69 \%$ of water maser sites are associated with star formation, $19 \%$ with evolved stars, and $12 \%$ with unknown identification. We expect limited contamination of AGB stars coincident with our GBT pointings because the observations are targeted toward the clump peak $S_{1.1 \mathrm{~mm}}$ and $N$ $\left(\mathrm{H}_{2}\right)$ positions $\left(\theta_{\mathrm{HPBW}} \approx 30^{\prime \prime}\right)$ where a YSO is likely to be located, but a chance alignment by a field AGB star is unlikely. Thus, we use the detection of an $\mathrm{H}_{2} \mathrm{O}$ maser as an observational indicator of star formation activity.

Large observational $\mathrm{H}_{2} \mathrm{O}$ maser survey programs have been carried out by the Arcetri survey (Valdettaro et al. 2001), HOPS (Walsh et al. 2011), and the RMS $\mathrm{H}_{2} \mathrm{O}$ Maser Survey (Urquhart et al. 2011). The variability of $\mathrm{H}_{2} \mathrm{O}$ masers makes multiple catalogs at different epochs complementary for systems that drop below a survey's sensitivity limits (Furuya et al. 2003). The Arcetri catalog aggregates observations of all known literature $\mathrm{H}_{2} \mathrm{O}$ water masers prior to 2001. HOPS is a blind Galactic plane survey with overlap coverage with the BGPS extending to $\ell<20^{\circ}$. The HOPS team discovered a number of new $\mathrm{H}_{2} \mathrm{O}$ masers not found in the Arcetri catalog, most in the southern sky, which is inaccessible to the Arcetri survey. While the survey overlap between HOPS and the BGPS GBT $\mathrm{H}_{2} \mathrm{O}$ maser survey is limited to $10^{\circ}<\ell<20^{\circ}$, it provides valuable context to evaluate the targeted GBT observations. In this range, the GBT observations have a 3.4 times higher detection rate toward BGPS clumps. This is likely due to the tighter beam coupling of the GBT compared to Mopra and the lower baseline RMS for the targeted, singlepointing observing strategy. The GBT observations primarily detect weaker $\mathrm{H}_{2} \mathrm{O}$ masers with median integrated intensity of $\sim 5 \mathrm{Jy} \mathrm{km} \mathrm{s}^{-1}$ compared to HOPS with $\sim 5 \times 10^{2} \mathrm{Jy} \mathrm{km} \mathrm{s}^{-1}$.

\subsection{2. $\mathrm{CH}_{3} \mathrm{OH}$ Masers}

The $6.7 \mathrm{GHz}$ Class II methanol $\left(\mathrm{CH}_{3} \mathrm{OH}\right)$ maser is thought to be exclusively associated with high-mass star formation because of the environments necessary for IR pumping (Breen et al. 2013). A comparison between $6.7 \mathrm{GHz} \mathrm{CH}_{3} \mathrm{OH}$ maser emission from the MMB and ATLASGAL $870 \mu \mathrm{m}$ dust emission finds that $99 \%$ of MMB sources are associated with ATLASGAL emission, indicating that methanol maser emission is almost ubiquitously associated with massive protostellar clumps (Urquhart et al. 2015). We associate BGPS clumps with $\mathrm{CH}_{3} \mathrm{OH}$ maser indicators based on available literature data. We use data from the Arecibo $\mathrm{CH}_{3} \mathrm{OH}$ survey (Pandian et al. 2007, 2011), MMB (Breen et al. 2012a, 2012b, 2014, 2015), and aggregated literature data in Pestalozzi et al. (2005). Within the overlap region, MMB data extend from $10^{\circ}<\ell<60^{\circ}$, Arecibo data extend from $35^{\circ}<\ell<54^{\circ}$, and the data in Pestalozzi et al. (2005) are all-sky targeted observations. Thus, there is a small gap in the $\mathrm{CH}_{3} \mathrm{OH}$ maser survey data from $60^{\circ}<\ell<65^{\circ}$, but there are only 15 BGPS clumps in this range, so there should not exist a strong sampling bias. While the MMB is the most uniform and complete of the surveys, multiple overlapping data sets are useful because the intensity of $\mathrm{CH}_{3} \mathrm{OH}$ masers is time variable. This can be important for masers that drop below the sensitivity limits of a single survey. With multiple surveys at different epochs we create a more complete catalog of $\mathrm{CH}_{3} \mathrm{OH}$ masers.

\subsection{Radio Continuum-UCH II Regions}

$\mathrm{UCH}$ II regions are an unambiguous indicator of high-mass star formation because an OB star must be present to internally photoionize the clump. We compare BGPS with CORNISH (Hoare et al. 2012; Purcell et al. 2013) observed with the JVLA in $5 \mathrm{GHz}$ continuum from $10^{\circ}<\ell<65^{\circ}$. We compare all 241 sources classified as UCH II region candidates in Purcell et al. (2013), excluding 43 sources classified as either "H II-Region," "Diffuse H II-Region," or "Dark H II-Region," representative of later stages of evolution. We find 219/243 (90.1\%) CORNISH UCH II candidates associated with 170 BGPS clumps. No BGPS clumps are exclusively identified with star formation activity from the CORNISH UCH II region sources. All such clumps are associated with an additional evolutionary indicator: $169 \mathrm{Hi}-\mathrm{GAL} 70 \mu \mathrm{m}, 69$ mid-IR, $103 \mathrm{H}_{2} \mathrm{O}$ maser, and 70 $\mathrm{CH}_{3} \mathrm{OH}$ maser. Urquhart et al. (2013b) compare CORNISH with ATLASGAL and find that 213/243 are matched to 170 ATLASGAL clumps. The $24 \mathrm{CORNISH}$ sources classified as $\mathrm{UCH}$ II region candidates that are not associated with millimeter continuum emission in the BGPS could potentially be BGPS nondetections, contamination/misidentification in the CORNISH survey, or more evolved systems that have disrupted most of the surrounding clump.

\subsection{Star Formation Indicator Catalog Summary}

\subsubsection{Star Formation Indicator Statistics}

We combine these catalogs of observational indicators of star formation activity into a series of flags to sort clumps into groups for joint analysis of their physical properties. The most basic distinction is between clumps that are associated with no star formation indicators, the SCCs, and "protostellar" clumps that are associated with one or more indicator. The star formation indicator groups are then ranked by the approximate luminosity of a single protostar that produces the indicator (see Figure 5). These rankings are a heuristic motivated by proposed linear clump evolutionary sequences (Chambers et al. 2009; Battersby et al. 2010, 2011), but the ordering is settled upon 
because it consistently yields monotonic trends for clump properties. "70 $\mu \mathrm{m}$ Unique" are clumps that only contain a HiGAL compact source and thus are candidates for deeply embedded $(\approx$ Class $0 /$ I) protostellar activity. "Mid-IR" clumps are associated with one or more R08 YSO-flagged source, RMS protostellar source, and/or EGO. We group R08, RMS, and EGOs together because they are frequently coincident with the same protostellar sources and they use similar wavelengths to identify activity. " $\mathrm{H}_{2} \mathrm{O}$ " clumps are associated with one or more $\mathrm{H}_{2} \mathrm{O}$ masers from the BGPS GBT survey, Arcetri, and/or HOPS. Similarly, " $\mathrm{CH}_{3} \mathrm{OH}$ " clumps are associated with one or more $\mathrm{CH}_{3} \mathrm{OH}$ masers from Pestalozzi et al. (2005), Arecibo, and/or MMB. "UCH II" clumps are associated with the CORNISH UCH II regions. In Section 5 we will use these categories to compare physical properties.

Table 4 lists the detection statistics for sources matched to the BGPS in the overlap region and the number of clumps matched to sources. We find that in the common overlap region $2223 / 4683(47.5 \%)$ are starless candidates and 2460/ $4683(52.5 \%)$ show some indicator of protostellar activity. This fraction of SCCs is lower than the upper limit of $67 \%$ estimated from the ATLASGAL survey by Csengeri et al. (2014), which compares ATLASGAL clumps to WISE $22 \mu \mathrm{m}$ and MSX $21 \mu \mathrm{m}$ point-source catalogs. The smaller fraction of SCCs in our analysis is likely due to the inclusion of protostellar indicators with lower-luminosity sensitivity such as Herschel $70 \mu \mathrm{m}$ sources in our analysis. The Herschel $70 \mu \mathrm{m}$ sources account for the largest fraction of star formation indicators detected toward protostellar clumps (2168/2460 [88\%] of protostellar clumps contain a $70 \mu \mathrm{m}$ source flag). No strong variation in the Galactic longitude distribution is observed between SCCs and protostellar clumps (Figure 5). Individual star formation indicator flags for each clump are listed in Table 5.

Except for $70 \mu \mathrm{m}$ unique, the BGPS indicator groups are not mutually exclusive. Indeed, 70/170 (41\%) of $\mathrm{UCH}_{\text {II }}$ clumps are also associated with a $\mathrm{CH}_{3} \mathrm{OH}$ maser and 103/170 (61\%) are associated with an $\mathrm{H}_{2} \mathrm{O}$ maser. There are no BGPS clumps uniquely associated with $\mathrm{H}_{2} \mathrm{O}, \mathrm{CH}_{3} \mathrm{OH}$, or $\mathrm{UCH}$ II flags; those clumps are always associated with another protostellar indicator. This fact indicates that it is unlikely that a population of $\mathrm{H}_{2} \mathrm{O}$ unique clumps (meaning that $\mathrm{H}_{2} \mathrm{O}$ masers are the only indicator of star formation activity) exist toward clumps that have been targeted by our GBT observations (Sections 2.2 and 3.3.1). Figure 6 shows the intersection between indicators with the fraction of the total calculated for each. For example, 70 clumps are associated with both $\mathrm{CH}_{3} \mathrm{OH}$ masers and $\mathrm{UCH}$ II regions: $0.412(70 / 170)$ of those with $\mathrm{UCH}$ II regions are also associated with $\mathrm{CH}_{3} \mathrm{OH}$ masers; alternatively, 0.235 (70/298) of those with $\mathrm{CH}_{3} \mathrm{OH}$ masers are also associated with $\mathrm{UCH}$ II regions.

Among these indicators, the $\mathrm{CH}_{3} \mathrm{OH}$ and $\mathrm{UCH}$ II clumps are the most consistent indicators of high-mass protostellar activity. However, it is important to note that more extreme indicators of star formation activity do not necessarily represent a later stage in a linear clump evolutionary sequence. For one, not all clumps will have sufficient mass to form an $\mathrm{OB}$ star to drive an observable $\mathrm{CH}_{3} \mathrm{OH}$ maser or $\mathrm{UCH}$ II region. The sequence of star formation indicators outlined in Battersby et al. (2010) is consistent with the expected sequence of observational indicators for a high-mass protostellar core, but BGPS clumps are hierarchical constructs of density, likely composed of multiple cores (Merello et al. 2015). The surveys also do not have high enough resolution to identify single protostellar sources at large distances. For example, what appears to be a Hi-GAL compact source could be a protocluster at several kiloparsecs. In this case, the only certain true evolutionary transition is from the starless clump phase to the protostellar phase.

\subsubsection{Comparison with Published Starless Clump Catalogs}

We compare our protostellar indicator flags to the previously published starless clump catalog of Tackenberg et al. (2012), which identified 210 starless candidates with peak column density $N\left(\mathrm{H}_{2}\right)>10^{23} \mathrm{~cm}^{-2}$ from ATLASGAL $870 \mu$ m images in the $10^{\circ}<l<20^{\circ}$ range. Tackenberg et al. (2012) use a combination of mid-IR colors from Spitzer imaging plus visual analysis of $24 \mu \mathrm{m}$ point sources to identify starless candidates. Within this longitude range, there are 52 BGPS catalog sources with peak positions that lie within the BGPS angular resolution $\left(33^{\prime \prime}\right)$ of the Tackenberg et al. (2012) starless candidate position. We find that 30 out of those 52 clumps $(58 \%)$ contain an indicator of star formation activity in our catalog. Nearly all of those associations result from identification of a $70 \mu \mathrm{m}$ source in our catalog. This result highlights the necessity of far-IR imaging to identify deeply embedded and lowerluminosity YSOs.

We also compare to the Traficante et al. (2015a) catalog of 1684 Hi-GAL clumps associated with IRDCs in the $10^{\circ}<\ell<55^{\circ}$ and $|b| \leqslant 1^{\circ}$ range. Clumps are extracted from the IRDC catalog of Peretto \& Fuller (2009). A novel algorithm (Hyper; Traficante et al. 2015b) is used for clump extraction and photometry, and counterparts at $70 \mu \mathrm{m}$ are used for protostellar identification. When compared by positions, 963 clumps in Traficante et al. (2015a) are associated with 692 BGPS clumps (14.8\% of the BGPS clumps in the overlap region), whereas the remaining 721 clumps are not associated. Among the 692 BGPS clumps with associations, $550(80.3 \%)$ star-forming categorizations agree and 142 (19.7\%) conflict. The conflicts are dominated (109 of 142 conflicts) by cases of a smaller "subcomponent" starless clump in the Traficante et al. (2015a) catalog associated with a larger "parent" BGPS clump with a protostellar indicator (N.B. the Herschel Hi-GAL resolution at $160 \mu \mathrm{m}$ is 13 !' 6 , which is 2.4 times higher resolution than the BGPS at $1.1 \mathrm{~mm})$. A minority of $33(4.8 \%)$ BGPS clumps remain that we have classified as starless candidates that contain $70 \mu \mathrm{m}$ counterparts from the Traficante et al. (2015a) catalog. In these cases we have conservatively assigned Hi-GAL flag 3's (28/33) for ambiguous high backgrounds in the visual inspection.

\section{DETERMINING HELIOCENTRIC DISTANCE}

\subsection{Distance Probability Density Functions}

A radial velocity measurement for Galactic positions within the solar circle yields a kinematic ambiguity between a near and far distance. Unique radial velocities are determined from observations of dense gas tracers $\left(\mathrm{HCO}^{+}, \mathrm{N}_{2} \mathrm{H}^{+}\right.$, or $\mathrm{NH}_{3}$; Dunham et al. 2011b; Shirley et al. 2013) and from morphologically matching the BGPS clumps to the ${ }^{13} \mathrm{CO}$ spectra from the GRS (see Ellsworth-Bowers et al. 2015). A Bayesian statistical methodology of formulating clump DPDFs based on a range of prior distributions has been carried out and applied to the BGPS by Ellsworth-Bowers et al. (2013, 2015). 
Table 5

Star Formation Indicator Flags

\begin{tabular}{|c|c|c|c|c|c|c|c|c|c|c|c|c|c|c|c|c|c|c|c|}
\hline \multirow{2}{*}{$\begin{array}{l}\text { ID } \\
\text { Number }\end{array}$} & \multicolumn{2}{|c|}{ Activity } & \multirow{2}{*}{$\begin{array}{c}\text { Hi-GAL } \\
\text { Flag }\end{array}$} & \multicolumn{2}{|c|}{ MIPS } & \multicolumn{4}{|c|}{ Mid-IR } & \multicolumn{4}{|c|}{$\mathrm{H}_{2} \mathrm{O}$} & \multicolumn{4}{|c|}{$\mathrm{CH}_{3} \mathrm{OH}$} & \multicolumn{2}{|c|}{$\mathrm{UCH}$ II } \\
\hline & SF? & $P_{\text {proto }}$ & & Cat. & Arch. & IR? & R08 & RMS & EGO & $\mathrm{H}_{2} \mathrm{O}$ ? & GBT & Arc & HOPS & $\mathrm{CH}_{3} \mathrm{OH}$ ? & MMB & Pesta. & Pand. & UCHII? & Corn. \\
\hline 5096 & 1 & 1.00 & 2 & 5 & 5 & 1 & 1 & 0 & 0 & 1 & 1 & 0 & 0 & 1 & 2 & 0 & 0 & 1 & 1 \\
\hline 5097 & 1 & 1.00 & 4 & 2 & 1 & 1 & 1 & 0 & 0 & 0 & 0 & 0 & 0 & 0 & 0 & 0 & 0 & 0 & 0 \\
\hline 5098 & 1 & 1.00 & 1 & 5 & 7 & 1 & 1 & 0 & 0 & 0 & 0 & 0 & 0 & 0 & 0 & 0 & 0 & 0 & 0 \\
\hline 5099 & 1 & 0.50 & 3 & 2 & 1 & 1 & 1 & 0 & 0 & 0 & 0 & 0 & 0 & 0 & 0 & 0 & 0 & 0 & 0 \\
\hline 5100 & 1 & 1.00 & 1 & 1 & 2 & 1 & 1 & 0 & 0 & 0 & 0 & 0 & 0 & 0 & 0 & 0 & 0 & 0 & 0 \\
\hline 5101 & 1 & 1.00 & 2 & 2 & 8 & 1 & 1 & 0 & 0 & 0 & 0 & 0 & 0 & 1 & 0 & 1 & 0 & 0 & 0 \\
\hline 5102 & 1 & 1.00 & 1 & 3 & 6 & 1 & 1 & 0 & 0 & 0 & 0 & 0 & 0 & 0 & 0 & 0 & 0 & 0 & 0 \\
\hline 5103 & 1 & 1.00 & 2 & 2 & 8 & 2 & 0 & 0 & 0 & 0 & 0 & 0 & 0 & 0 & 0 & 0 & 0 & 0 & 0 \\
\hline 5104 & 0 & 0.00 & 0 & 2 & 3 & 0 & 0 & 0 & 0 & 0 & 0 & 0 & 0 & 0 & 0 & 0 & 0 & 0 & 0 \\
\hline 5105 & 0 & 0.00 & 0 & 0 & 0 & 0 & 0 & 0 & 0 & 0 & 0 & 0 & 0 & 0 & 0 & 0 & 0 & 0 & 0 \\
\hline
\end{tabular}

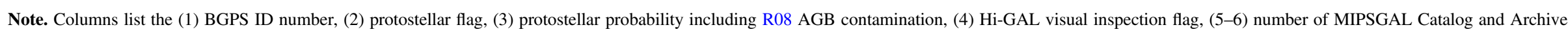

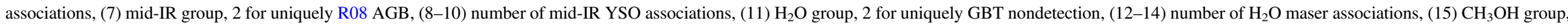
(16-18) number of $\mathrm{CH}_{3} \mathrm{OH}$ maser associations, (19) $\mathrm{UCH}$ II group, and (20) number of CORNISH associations.

References. see Section 3 for catalog descriptions.

(This table is available in its entirety in machine-readable form.) 


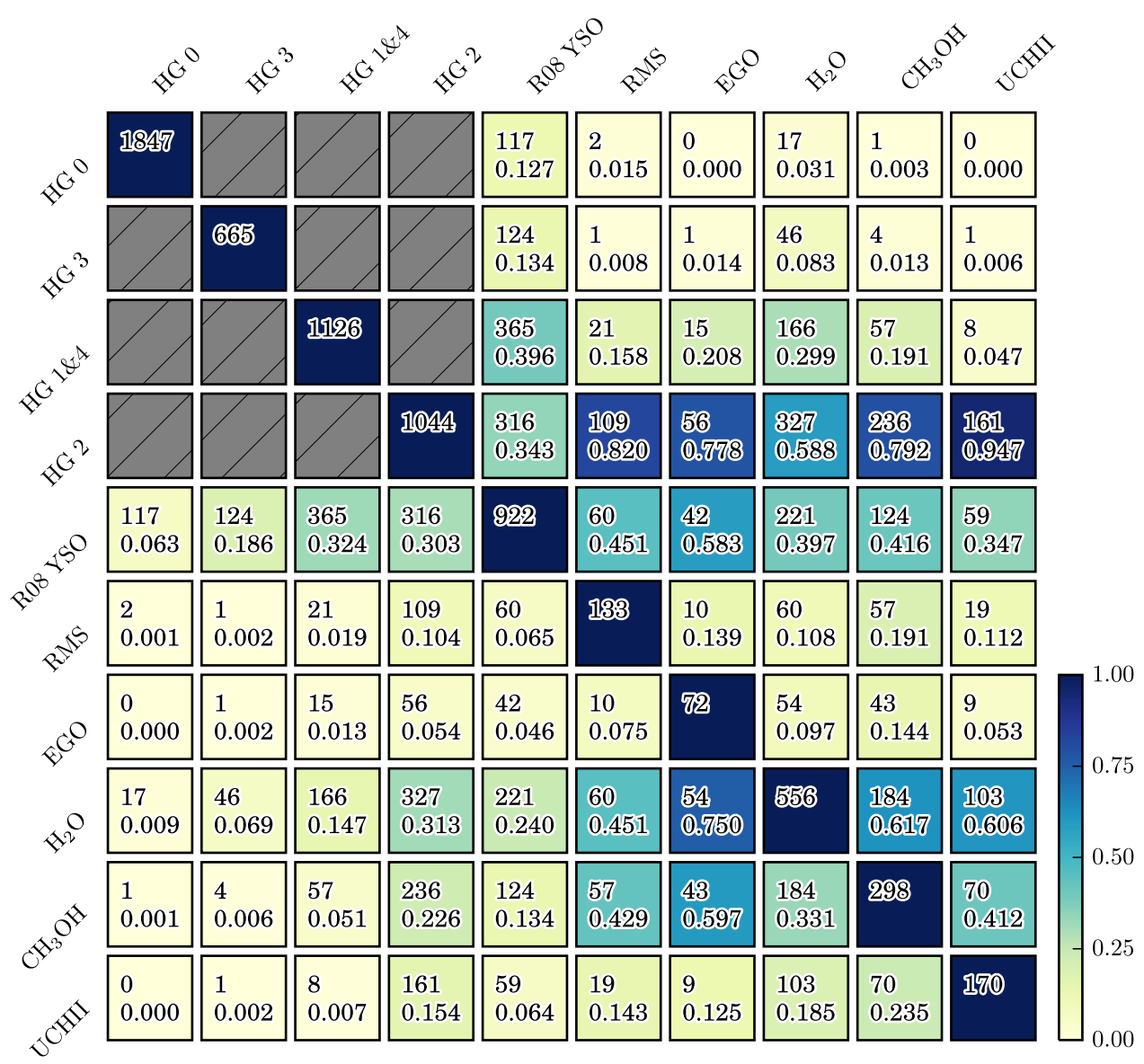

Figure 6. Intersection between pairs of star formation indicators. Totals for each indicator are shown diagonally. The top value in each square shows the number of overlapping indicators, and the bottom value shows the overlap fraction calculated with respect to the total of the column. The overlap fraction is color-coded by the scale on the right. To read, select the first indicator along the top axis, and drop down to the row of the second indicator along the left axis. The Hi-GAL flags are mutually exclusive for each clump and are grayed out.

Furthermore, to accurately propagate our uncertainty in heliocentric distance, we shall use MC random sampling of the DPDFs when calculating the derived physical properties. The DPDF is calculated by multiplying the likelihood function, derived from the measured $v_{\mathrm{LSR}}$ with a $7 \mathrm{~km} \mathrm{~s}^{-1}$ uncertainty to account for typical GMC dispersion and the Reid et al. (2014) rotation curve of the Galaxy, with prior distributions

$$
\operatorname{DPDF}\left(d_{\odot}\right)=\mathcal{L}\left(v_{\mathrm{LSR}}, l, b ; d_{\odot}\right) \prod_{i} P_{i}\left(d_{\odot}, l, b\right) .
$$

The priors are calculated from morphological matching to $8 \mu$ mabsorption features, latitude offsets compared to the Wolfire et al. (2003) model of the $\mathrm{H}_{2}$ distribution in the Galaxy, maser parallax measurements, and proximity to an $\mathrm{H}$ II region of known distance. The well-constrained DPDFs are defined to have full width of the $68 \%$ maximum-likelihood error bar $\mathrm{FW}_{68} \leqslant 2.3 \mathrm{kpc}$ (Ellsworth-Bowers et al. 2013). The total number of well-constrained DPDFs is 1710 in BGPS.

\subsection{Distance Resolution Broadcasting}

The well-constrained BGPS distance sample contains 1414 clump DPDFs representing $43 \%$ of the BGPS velocity catalog in $10^{\circ}<\ell<65^{\circ}$. Clumps show an easily distinguishable tendency to cluster in velocity coherent groups. Indeed, $75.4 \%$ of clumps in the overlap region have at least one adjacent neighbor (i.e., pixels touching) in the Bolocat label masks.
Clumps that are within spatial and kinematic proximity are more likely to be cospatial than chance alignments of noncospatial near/far kinematic distances. In order to increase the size of the distance sample, we apply a modified friends-offriends algorithm (Huchra \& Geller 1982) to associate or "broadcast" the known DPDFs based on whether clumps are nearby in $\left(\ell, b, v_{\mathrm{LSR}}\right)$ or PPV space. Because this technique uses only proximity in PPV space, the number of new associations does not have a strong selection effect from evolutionary stage or physical properties. To evaluate the optimal search angle-velocity search parameters, we use the distance sample as a training set and minimize the fraction of near/far misassociations.

We assign a clump to a "PPV group" or "group" by searching a distance $\rho$ around the clump in angular separation, $\theta_{s}$, and velocity, $v_{s}$ :

$$
\rho=\sqrt{\Delta \theta^{2}+\left(\frac{\theta_{s}}{v_{s}} \Delta v\right)^{2}}<\sqrt{2} \theta_{s} .
$$

Clumps within this neighborhood are assigned to a parent group, and the group is iteratively expanded by searching around the neighbors. This algorithm effectively creates groups out of a minimum density in PPV space. We calculate group assignments over a $30 \times 30$ grid of angular search and velocity search radii from $\theta_{s}=1 ! .8-10 ! 5$ and $v_{s}=1-6 \mathrm{~km} \mathrm{~s}^{-1}$. We use 
the known maximum-likelihood distances as a training set to evaluate the accuracy of the search parameters. We denote the conflict ratio, $R_{\text {conf }}$, as the number of clumps in groups with disagreeing distance resolutions divided by the number of clumps in groups with at least two distance resolutions. With $R_{\text {conf }}$ as a figure of merit, we select the search parameters that maximize the number of new distance resolutions for fixed $R_{\text {conf. It is important to note, however, that the DPDFs are not }}$ simply Boolean near/far kinematic distance resolutions. Thus,

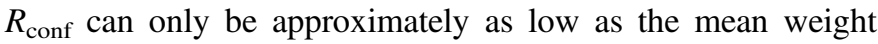
between the near/far probabilities in the DPDFs. EllsworthBowers et al. (2013) find that $8 \%$ of the DPDFs disagree with the ${ }^{13} \mathrm{CO}$ GRS KDA resolutions (KDARs) from Roman-Duval et al. (2009) using $\mathrm{H}_{\mathrm{I}}$ self-absorption (H ISA). We fix the conflict ratio to be reasonably consistent with this figure at $R_{\text {conf }}=0.12$ and select the search parameters $\left(\theta_{s}, v_{s}\right)=(3 ! 9$, $4.0 \mathrm{~km} \mathrm{~s}^{-1}$ ) that maximize new associations. In total, we associate 446 new DPDFs over the full survey and 226 in the survey overlap region. The KDA resolution broadcasting method thus increases the distance sample used in this study by $226(16 \%)$ to 1640 clumps in the overlap region.

The DPDFs added to the distance sample are constructed from the weighted priors of the DPDFs within the group. The optimized search parameters are used to associate the neighboring clumps in the group with a common kinematic near/far distance. To create a new posterior DPDF for a clump in a group, we apply a weighted average of all priors from the clumps in the group with a DPDF to the new group-member clump. The priors are given Gaussian weights by the PPVdistance $\rho$ with FWHM values set to the optimized $\theta_{s}$ and $v_{s}$ above. This weighting ensures that the closest clump priors in the group are most strongly weighted. To propagate our uncertainty in broadcasting the distance resolution of an individual clump, we apply a weighted error function (as a step-function analog) to the new posterior DPDF to account for $R_{\text {conf }}=0.12$. Down-weighting the distance resolutions by the conflict fraction accounts for the mean uncertainty in the group associations.

\subsection{Distance Comparisons}

Dunham et al. (2011b) used a combination of visually identified $8 \mu \mathrm{m}$ absorption features and $\mathrm{H}$ ISA to resolve the KDA toward a sample of BGPS clumps. Because these sources are also in our sample, we compare the distance resolutions in Dunham et al. (2011b) with the resolutions of the wellconstrained DPDFs in Ellsworth-Bowers et al. (2013) and Ellsworth-Bowers et al. (2015). The results of Dunham et al. (2011b) are based on the BGPS v1.0 data release and are assigned to BGPS v2.0 sources by mapping the peak flux positions of the v1.0 sources onto the v2.0 source masks. Of the 456 sources with resolved distances in Dunham et al. (2011b), 403 match to v2.0 sources and 100 have well-resolved KDAs in our sample. For sources flagged as either "near" or "tangent" in Dunham et al. (2011b), we find that $69 / 78 \approx 88 \%$ agree. For sources flagged as "far" that had no incidence of an $8 \mu \mathrm{m}$ absorption feature or $\mathrm{HISA}$, we find that only $11 /$ $20 \approx 53 \%$ agree.

The lack of an H ISA feature is commonly used to associate GMCs with the far kinematic distance (Roman-Duval et al. 2010), where the superposition against the background

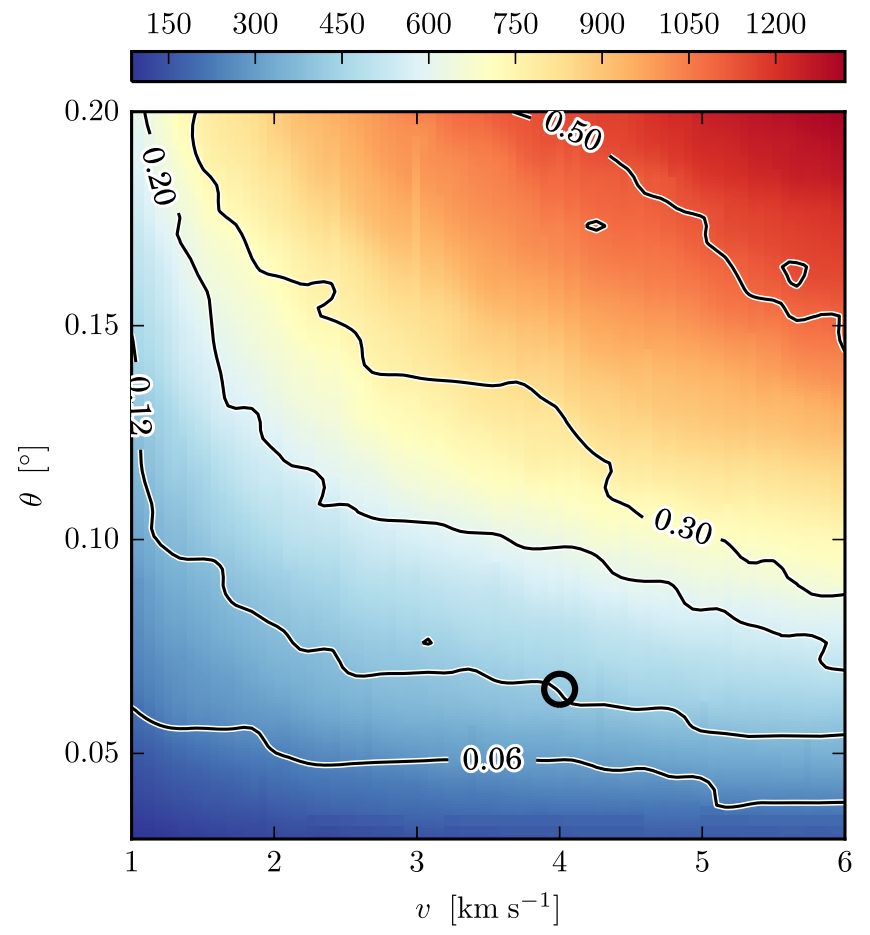

Figure 7. Optimization of the velocity and angular search parameters for the number of new clump KDA resolutions (color scale). The new KDA resolutions are in PPV groups with one or more resolved KDA member that have a well-constrained DPDF. Black contours show the conflict ratios $R_{\text {conf }}$, or the ratio of clumps in PPV groups with conflicting near/far KDA resolutions to the total number of clumps in PPV groups with two or more distance-resolved members. Maximizing the number of new KDAR associations for a fixed $R_{\text {conf }}=0.12$ gives 446 new distance resolutions at $\theta=0.065(3 ! 9)$ and $v=4.0 \mathrm{~km} \mathrm{~s}^{-1}$ (black circle).

$\mathrm{H}$ I distribution produces absorption at the velocity coincident with the high column density present in the GMC. The clumps found in the BGPS are both on smaller spatial scales and at higher spatial densities than GMCs. The disagreement for far distance resolutions for clumps is because the $\mathrm{H}$ I distribution does not follow that of the clump dust continuum, and thus the lack of an HiSA feature cannot be used to accurately resolve the KDA for BGPS clumps.

Wienen et al. (2015) use associations of ${ }^{13} \mathrm{CO}$ GRS isocontours in PPV space and the associated $\mathrm{HI}_{\mathrm{I}}$ absorption with those GRS clouds to resolve $44 \%$ of ATLASGAL clumps to the far kinematic distance. This is twice the $22 \%$ fraction found in Ellsworth-Bowers et al. (2015). Comparison of the Wienen et al. (2015) KDA resolutions with Ellsworth-Bowers et al. (2015) DPDFs indicates a 30\% conflict fraction. The origin of the larger conflict fraction is the larger PPV spaces used to associate objects in the Wienen et al. (2015) paper. Figure 7 shows that the conflict fraction increases with angular separation and velocity for larger PPV groups. This result, combined with the larger number of far kinematic resolutions, will systematically skew distributions of physical properties such as $M, R, n$, and $\left\langle t_{\mathrm{ff}}\right\rangle$ to larger values for the Wienen et al. (2015) sample.

\section{ANALYSIS OF PHYSICAL PROPERTIES WITH STAR FORMATION INDICATORS}

In the following subsections, we shall compare and analyze observable and physical quantities for both the SCCs and protostellar clumps. For the protostellar clumps, we sort clumps 
Table 6

Clump Physical Properties

\begin{tabular}{|c|c|c|c|c|c|c|c|c|c|c|}
\hline \multirow{2}{*}{$\begin{array}{l}\text { ID } \\
\text { Number }\end{array}$} & \multicolumn{5}{|c|}{$d_{\odot}(\mathrm{pc})$} & \multicolumn{5}{|c|}{$M_{\mathrm{H}_{2}}^{\text {Total }}\left(\mathrm{M}_{\odot}\right)$} \\
\hline & 15.9 & 50.0 & 84.1 & ML & $\sigma_{1 / 2}$ & 15.9 & 50.0 & 84.1 & ML & $\sigma_{1 / 2}$ \\
\hline 2409 & 8073 & 8630 & 9264 & 8711 & 403.2 & 3863 & 4597 & 5522 & 4547 & 553.2 \\
\hline 2418 & 4602 & 5036 & 5581 & 5187 & 329.1 & 904.2 & 1107 & 1369.6 & 962.5 & 150.4 \\
\hline 2421 & 4603 & 5046 & 5578 & 4899 & 322.0 & 1318 & 1617 & 2016 & 1455.5 & 229.1 \\
\hline 2424 & 4595 & 5042 & 5577 & 4852 & 328.1 & 3763 & 4575 & 5662 & 4842 & 625.4 \\
\hline 2425 & 4594 & 5037 & 5576 & 4800 & 332.3 & 3147 & 3830 & 4744 & 3888 & 529.0 \\
\hline 2433 & 2297 & 3047 & 3914 & 2882 & 502.3 & 276.9 & 490.9 & 822.9 & 425.5 & 164.0 \\
\hline 2434 & 2369 & 3158 & 3964 & 2732 & 500.0 & 389.4 & 687.1 & 1120.7 & 564.8 & 226.3 \\
\hline 2436 & 2443 & 3156 & 3786 & 3113 & 443.6 & 157.7 & 304.0 & 533.3 & 276.5 & 118.88 \\
\hline
\end{tabular}

Note. Physical properties calculated from the MC simulations for each clump in the distance sample. The percentiles of the posterior distributions (15.9-84.1), maximum likelihood (ML), and median absolute deviation $\left(\sigma_{1 / 2}\right)$ with uncertainties are calculated. Columns list the (1) BGPS ID number, (2-4) heliocentric distance, (5-8) total mass. A subset of the derived physical properties $\left(d_{\odot}, M\right)$ are shown above for guidance regarding the table's form and content.

(This table is available in its entirety in machine-readable form.)

by their star formation indicators into subsamples described in Section 3.5. As noted in Section 3, this ordering is not an evolutionary sequence but is roughly ordered by the typical luminosity required to produce each indicator. In the following figures, the top two panels indicate the SCC and protostellar candidate subsamples, while subsequent panels indicate various star formation indicator subsamples. For the physical properties and sampling techniques described below, Table 6 lists the derived physical properties and uncertainties for each clump in the distance sample, and Table 7 lists the statistical properties and uncertainties for subsamples of clumps sorted by star formation indicator.

\subsection{Sampling}

For each subsample we compute MC simulations to randomly sample all of the input parameters, both measured quantities with statistical uncertainties (e.g., $S_{\nu}, T_{\mathrm{K}}$ ) and DPDFs. By calculating descriptive statistical quantities (e.g., $\mu_{1 / 2}, \rho_{\text {spear }}$ ) from suites of $\mathrm{MC}$ simulations, we can estimate the uncertainty and significance of comparisons between samples of clumps associated with different star formation indicators. The suites of MC simulations also more accurately propagate the uncertainties when compared to simply creating a single histogram of the maximum-likelihood values, because some quantities such as heliocentric distance can have highly asymmetric and/or bimodal PDFs. Thus, while a single clump may have large fractional uncertainties in any given derived physical property, we can make robust and accurate calculations for large $\left(\sim 10^{2}-10^{3}\right)$, statistically significant subsamples of clumps.

\subsubsection{AGB Contamination Resampling}

Contamination from AGB stars is a significant source of uncertainty when the only indicator of star formation activity within a clump is from the mid-infrared colors of a point source. In order to propagate this uncertainty due to AGB contamination, we resample flags discriminating between R08 YSO and AGB sources in the MC simulations. We use conservative estimates of the contamination fractions between the R08 YSO contaminants in the AGB classification and AGB contaminants in the YSO classification. As described in Section 3.2.2, we apply contamination fractions of $40 \%$ for AGB sources and 50\% for YSO sources. All R08 sources within clumps are resampled this way. If a clump is associated with another star formation indicator besides R08, then it is still flagged as protostellar and the MC flag resampling will not affect the clump's overall designation. In the overlap region, only 280 clumps $(6.0 \%)$ are uniquely associated with R08 sources, and 122 clumps (7.4\%) in the distance sample. Resampling propagates our uncertainty in the R08 flags, but because of significant coincidence with other star formation indicators, it introduces only a minor effect in discriminating between SCCs and protostellar clumps. In the following analysis, AGB contamination resampling is used in all MC calculations.

\subsubsection{DPDF Sampling and Distance Biases}

For the calculation of quantities that are distance dependent (e.g., $R, M$ ), we draw the heliocentric distance from the source DPDF. Each MC simulation consists of $10^{4}$ random draws. Since the output distributions of the physical properties may be skewed, we use robust statistical indicators such as the median $\left(\mu_{1 / 2}\right)$ and median absolute deviation $\left(\sigma_{1 / 2}\right)^{12}$ as conservative characterizations of the distribution of properties for a particular subsample.

Before we compare physical properties among clumps with different star formation indicators (Sections 5.2-5.4), we compare the distribution of DPDFs for each star formation indicator subsample defined in Section 3.5. Figure 8(c) shows the mean distribution or average DPDF for each indicator. The indicator categories show similar distributions of heliocentric distance. While the number of clumps associated with each indicator varies, the range and distribution of $d$ are similar, with medians between 4.4 and $5.1 \mathrm{kpc}$. As a result, physical properties that depend linearly or quadratically on distance will not be strongly biased by the underlying distance distribution for each indicator subsample. Sources with

\footnotetext{
12 The median absolute deviation is defined by $\sigma_{1 / 2}=$ $\mu_{1 / 2, i}\left(\left|X_{i}-\mu_{1 / 2, j}\left(X_{j}\right)\right|\right)$ for a univariate data set $X$. For normally distributed data, $\sigma_{1 / 2}$ is related to the standard deviation by $\sigma \approx 1.48260 \sigma_{1 / 2}$.
} 
Table 7

Statistics of Observed and Physical Properties

\begin{tabular}{|c|c|c|c|c|c|c|c|c|}
\hline Property & Indicator & $N$ & 10 & 25 & $50\left(\mu_{1 / 2}\right)$ & 75 & 90 & $\sigma_{1 / 2}$ \\
\hline$\overline{\Omega^{\text {Total }}}$ & Starless Cand. & 2223 & $920.2_{-3.5}^{+3.7}$ & $1824.2_{-4.7}^{+4.7}$ & $3620.4_{-8.3}^{+8.2}$ & $6531_{-17}^{+17}$ & $10668_{-39}^{+38}$ & $2110.9_{-6.2}^{+6.2}$ \\
\hline \multirow[t]{6}{*}{ (sq. $\operatorname{arcsec}$ ) } & Protostellar & 2460 & $2002.9_{-6.6}^{+6.5}$ & $3678.3_{-10}^{+9.8}$ & $7114_{-16}^{+15}$ & $13755_{-36}^{+35}$ & $23286_{-64}^{+65}$ & $4191_{-12}^{+12}$ \\
\hline & $70 \mu \mathrm{m}$ Unique & 995 & $1629.6_{-7.0}^{+7.0}$ & $2908_{-11}^{+11}$ & $4985_{-17}^{+17}$ & $9148_{-28}^{+28}$ & $14540_{-64}^{+63}$ & $2626_{-12}^{+12}$ \\
\hline & Mid IR & 1022 & $2360_{-14}^{+14}$ & $4575_{-15}^{+15}$ & $8802_{-42}^{+42}$ & $17114_{-63}^{+59}$ & $30320_{-130}^{+130}$ & $5416_{-22}^{+22}$ \\
\hline & $\mathrm{H}_{2} \mathrm{O}$ & 556 & $3129_{-17}^{+16}$ & $5912_{-36}^{+35}$ & $12445_{-48}^{+49}$ & $23076_{-74}^{+78}$ & $36950_{-230}^{+240}$ & $7632_{-36}^{+36}$ \\
\hline & $\mathrm{CH}_{3} \mathrm{OH}$ & 298 & $3792_{-23}^{+23}$ & $7330_{-47}^{+49}$ & $15526_{-75}^{+77}$ & $27840_{-160}^{+160}$ & $40420_{-290}^{+270}$ & $9135_{-66}^{+67}$ \\
\hline & UCH II & 170 & $4661_{-44}^{+42}$ & $9635_{-54}^{+57}$ & $18060_{-110}^{+120}$ & $31190_{-180}^{+170}$ & $45480_{-330}^{+340}$ & $10238_{-120}^{+95}$ \\
\hline \multirow[t]{7}{*}{$\Omega^{\text {Total }} / \Omega^{\text {FWHM }}$} & Starless Cand. & 2103 & $1.1517_{-0.0016}^{+0.0016}$ & $1.2830_{-0.0016}^{+0.0016}$ & $1.5534_{-0.0026}^{+0.0026}$ & $2.0949_{-0.0042}^{+0.0043}$ & $3.088_{-0.012}^{+0.012}$ & $0.3326_{-0.0018}^{+0.0019}$ \\
\hline & Protostellar & 2426 & $1.2676_{-0.0023}^{+0.0023}$ & $1.6210_{-0.0034}^{+0.0033}$ & $2.5959_{-0.0055}^{+0.0054}$ & $5.485_{-0.014}^{+0.015}$ & $14.191_{-0.052}^{+0.050}$ & $1.2064_{-0.0044}^{+0.0045}$ \\
\hline & $70 \mu \mathrm{m}$ Unique & 975 & $1.1915_{-0.0028}^{+0.0028}$ & $1.4324_{-0.0039}^{+0.0039}$ & $1.9578_{-0.0061}^{+0.0059}$ & $3.1400_{-0.0096}^{+0.0097}$ & $5.167_{-0.024}^{+0.024}$ & $0.6559_{-0.0047}^{+0.0047}$ \\
\hline & Mid IR & 1010 & $1.3419_{-0.0045}^{+0.0046}$ & $1.7800_{-0.0047}^{+0.0048}$ & $3.030_{-0.010}^{+0.010}$ & $7.570_{-0.032}^{+0.032}$ & $16.923_{-0.082}^{+0.081}$ & $1.6073_{-0.0094}^{+0.0092}$ \\
\hline & $\mathrm{H}_{2} \mathrm{O}$ & 555 & $1.886_{-0.011}^{+0.011}$ & $3.350_{-0.018}^{+0.019}$ & $7.142_{-0.047}^{+0.048}$ & $16.566_{-0.080}^{+0.077}$ & $39.33_{-0.25}^{+0.24}$ & $4.906_{-0.037}^{+0.037}$ \\
\hline & $\mathrm{CH}_{3} \mathrm{OH}$ & 297 & $2.709_{-0.017}^{+0.018}$ & $4.747_{-0.034}^{+0.035}$ & $11.215_{-0.057}^{+0.056}$ & $25.16_{-0.16}^{+0.16}$ & $54.28_{-0.35}^{+0.35}$ & $7.643_{-0.056}^{+0.056}$ \\
\hline & UCH II & 170 & $4.066_{-0.037}^{+0.035}$ & $8.501_{-0.058}^{+0.054}$ & $16.377_{-0.085}^{+0.085}$ & $34.87_{-0.25}^{+0.25}$ & $59.61_{-0.49}^{+0.46}$ & $10.906_{-0.080}^{+0.080}$ \\
\hline \multirow{7}{*}{$\begin{array}{l}S_{1.1}^{\text {Total }} \\
\text { (Jy) }\end{array}$} & Starless Cand. & 2223 & $0.1021_{-0.0030}^{+0.0031}$ & $0.1891_{-0.0033}^{+0.0033}$ & $0.3559_{-0.0046}^{+0.0046}$ & $0.6729_{-0.0084}^{+0.0087}$ & $1.275_{-0.018}^{+0.018}$ & $0.2034_{-0.0037}^{+0.0036}$ \\
\hline & Protostellar & 2460 & $0.2590_{-0.0056}^{+0.0055}$ & $0.4639_{-0.0064}^{+0.0064}$ & $0.9493_{-0.0099}^{+0.0098}$ & $2.229_{-0.025}^{+0.024}$ & $5.256_{-0.065}^{+0.063}$ & $0.6093_{-0.0083}^{+0.0079}$ \\
\hline & $70 \mu \mathrm{m}$ Unique & 995 & $0.2058_{-0.0070}^{+0.0009}$ & $0.3436_{-0.0071}^{+0.00072}$ & $0.6090_{-0.0099}^{+0.010}$ & $1.101_{-0.017}^{+0.0017}$ & $1.928_{-0.036}^{+0.037}$ & $0.3220_{-0.0079}^{+0.0079}$ \\
\hline & Mid IR & 1022 & $0.320_{-0.011}^{+0.011}$ & $0.624_{-0.013}^{+0.013}$ & $1.348_{-0.021}^{+0.021}$ & $3.448_{-0.049}^{+0.049}$ & $8.26_{-0.16}^{+0.16}$ & $0.929_{-0.018}^{+0.018}$ \\
\hline & $\mathrm{H}_{2} \mathrm{O}$ & 556 & $0.516_{-0.018}^{+0.019}$ & $1.050_{-0.030}^{+0.031}$ & $2.579_{-0.049}^{+0.048}$ & $6.71_{-0.12}^{+0.12}$ & $14.29_{-0.29}^{+0.30}$ & $1.917_{-0.041}^{+0.042}$ \\
\hline & $\mathrm{CH}_{3} \mathrm{OH}$ & 298 & $0.600_{-0.030}^{+0.031}$ & $1.326_{-0.042}^{+0.043}$ & $3.363_{-0.072}^{+0.0068}$ & $9.09_{-0.19}^{+0.21}$ & $18.52_{-0.48}^{+0.50}$ & $2.519_{-0.062}^{+0.059}$ \\
\hline & UCH II & 170 & $0.896_{-0.048}^{+0.047}$ & $2.115_{-0.097}^{+0.097}$ & $4.63_{-0.12}^{+0.12}$ & $11.37_{-0.29}^{+0.29}$ & $19.78_{-0.57}^{+0.46}$ & $3.562_{-0.099}^{+0.10}$ \\
\hline \multirow{7}{*}{$\begin{array}{l}S_{1.1}^{\mathrm{FWHM}} \\
(\mathrm{Jy})\end{array}$} & Starless Cand. & 2223 & $0.0619_{-0.0028}^{+0.0028}$ & $0.1442_{-0.0029}^{+0.0029}$ & $0.2871_{-0.0039}^{+0.0038}$ & $0.5313_{-0.0063}^{+0.0066}$ & $0.963_{-0.014}^{+0.014}$ & $0.1714_{-0.0030}^{+0.0030}$ \\
\hline & Protostellar & 2460 & $0.1911_{-0.0043}^{+0.0043}$ & $0.3425_{-0.0044}^{+0.0045}$ & $0.6316_{-0.0061}^{+0.0061}$ & $1.234_{-0.011}^{+0.011}$ & $2.332_{-0.025}^{+0.027}$ & $0.3611_{-0.0051}^{+0.0051}$ \\
\hline & $70 \mu \mathrm{m}$ Unique & 995 & $0.1515_{-0.0055}^{+0.0056}$ & $0.2610_{-0.0056}^{+0.0055}$ & $0.4417_{-0.0070}^{+0.0069}$ & $0.751_{-0.012}^{+0.012}$ & $1.234_{-0.020}^{+0.021}$ & $0.2176_{-0.0055}^{+0.0056}$ \\
\hline & Mid IR & 1022 & $0.2344_{-0.0080}^{+0.00079}$ & $0.4396_{-0.0085}^{+0.0085}$ & $0.831_{-0.012}^{+0.0012}$ & $1.644_{-0.021}^{+0.021}$ & $3.179_{-0.048}^{+0.050}$ & $0.4908_{-0.0093}^{+0.0093}$ \\
\hline & $\mathrm{H}_{2} \mathrm{O}$ & 556 & $0.348_{-0.012}^{+0.012}$ & $0.612_{-0.013}^{+0.014}$ & $1.195_{-0.019}^{+0.019}$ & $2.502_{-0.039}^{+0.039}$ & $4.598_{-0.088}^{+0.088}$ & $0.722_{-0.017}^{+0.016}$ \\
\hline & $\mathrm{CH}_{3} \mathrm{OH}$ & 298 & $0.388_{-0.016}^{+0.017}$ & $0.687_{-0.020}^{+0.019}$ & $1.304_{-0.026}^{+0.028}$ & $2.901_{-0.056}^{+0.058}$ & $5.61_{-0.16}^{+0.17}$ & $0.812_{-0.026}^{+0.026}$ \\
\hline & UCH II & 170 & $0.533_{-0.030}^{+0.030}$ & $0.948_{-0.029}^{+0.029}$ & $1.664_{-0.042}^{+0.043}$ & $3.674_{-0.092}^{+0.092}$ & $6.99_{-0.21}^{+0.22}$ & $0.975_{-0.037}^{+0.039}$ \\
\hline \multirow{7}{*}{$\begin{array}{l}T_{\mathrm{K}} \\
(\mathrm{K})\end{array}$} & Starless Cand. & 248 & $11.685_{-0.085}^{+0.084}$ & $12.485_{-0.074}^{+0.073}$ & $13.961_{-0.091}^{+0.096}$ & $16.57_{-0.15}^{+0.16}$ & $21.64_{-0.36}^{+0.38}$ & $1.837_{-0.065}^{+0.064}$ \\
\hline & Protostellar & 1164 & $12.688_{-0.044}^{+0.045}$ & $14.081_{-0.042}^{+0.042}$ & $16.577_{-0.056}^{+0.055}$ & $21.62_{-0.13}^{+0.14}$ & $27.98_{-0.21}^{+0.18}$ & $3.133_{-0.052}^{+0.052}$ \\
\hline & $70 \mu \mathrm{m}$ Unique & 282 & $12.121_{-0.083}^{+0.074}$ & $12.989_{-0.072}^{+0.074}$ & $14.711_{-0.089}^{+0.080}$ & $17.48_{-0.19}^{+0.19}$ & $21.31_{-0.37}^{+0.44}$ & $1.976_{-0.074}^{+0.076}$ \\
\hline & Mid IR & 556 & $12.868_{-0.065}^{+0.003}$ & $14.116_{-0.057}^{+0.058}$ & $16.584_{-0.075}^{+0.075}$ & $21.82_{-0.24}^{+0.23}$ & $28.18_{-0.24}^{+0.32}$ & $3.011_{-0.071}^{+0.071}$ \\
\hline & $\mathrm{H}_{2} \mathrm{O}$ & 499 & $13.770_{-0.068}^{+0.067}$ & $15.508_{-0.067}^{+0.070}$ & $19.17_{-0.12}^{+0.12}$ & $25.76_{-0.16}^{+0.11}$ & $31.45_{-0.14}^{+0.19}$ & $4.353_{-0.080}^{+0.071}$ \\
\hline & $\mathrm{CH}_{3} \mathrm{OH}$ & 232 & $15.28_{-0.17}^{+0.17}$ & $17.84_{-0.14}^{+0.13}$ & $22.48_{-0.21}^{+0.21}$ & $27.33_{-0.20}^{+0.21}$ & $32.39_{-0.12}^{+0.22}$ & $4.74_{-0.11}^{+0.13}$ \\
\hline & $\mathrm{UCH}$ II & 134 & $18.02_{-0.36}^{+0.53}$ & $22.38_{-0.37}^{+0.24}$ & $27.27_{-0.18}^{+0.17}$ & $32.46_{-0.13}^{+0.40}$ & $39.88_{-0.84}^{+0.76}$ & $5.14_{-0.17}^{+0.21}$ \\
\hline \multirow{7}{*}{$\begin{array}{l}\sigma_{\mathrm{v}}\left(\mathrm{NH}_{3}\right) \\
\left(\mathrm{km} \mathrm{s}^{-1}\right)\end{array}$} & Starless Cand. & 319 & $0.3085_{-0.0067}^{+0.0061}$ & $0.4197_{-0.0051}^{+0.0051}$ & $0.5671_{-0.0054}^{+0.0058}$ & $0.882_{-0.010}^{+0.010}$ & $1.485_{-0.027}^{+0.030}$ & $0.2008_{-0.0048}^{+0.0052}$ \\
\hline & Protostellar & 1132 & $0.3998_{-0.0037}^{+0.0040}$ & $0.5369_{-0.0031}^{+0.0031}$ & $0.7160_{-0.0030}^{+0.0032}$ & $0.9862_{-0.0043}^{+0.0042}$ & $1.3305_{-0.010}^{+0.0083}$ & $0.2157_{-0.0026}^{+0.0027}$ \\
\hline & $70 \mu \mathrm{m}$ Unique & 297 & $0.3555_{-0.0063}^{+0.0048}$ & $0.4571_{-0.0049}^{+0.0054}$ & $0.6222_{-0.0058}^{+0.0061}$ & $0.8343_{-0.0057}^{+0.0067}$ & $1.077_{-0.019}^{+0.021}$ & $0.1819_{-0.0049}^{+0.0048}$ \\
\hline & Mid IR & 511 & $0.4002_{-0.0042}^{+0.0047}$ & $0.5256_{-0.0039}^{+0.0038}$ & $0.7132_{-0.0042}^{+0.0046}$ & $0.9699_{-0.0063}^{+0.0063}$ & $1.287_{-0.016}^{+0.002}$ & $0.2165_{-0.0032}^{+0.0032}$ \\
\hline & $\mathrm{H}_{2} \mathrm{O}$ & 493 & $0.5281_{-0.0050}^{+0.0044}$ & $0.6636_{-0.0033}^{+0.0033}$ & $0.8549_{-0.0050}^{+0.0050}$ & $1.1253_{-0.0087}^{+0.0083}$ & $1.469_{-0.016}^{+0.015}$ & $0.2197_{-0.0034}^{+0.0036}$ \\
\hline & $\mathrm{CH}_{3} \mathrm{OH}$ & 205 & $0.6210_{-0.0089}^{+0.0072}$ & $0.7528_{-0.0067}^{+0.0067}$ & $0.9851_{-0.0046}^{+0.0044}$ & $1.321_{-0.011}^{+0.0011}$ & $1.627_{-0.019}^{+0.036}$ & $0.2757_{-0.0080}^{+0.0066}$ \\
\hline & UCH II & 139 & $0.706_{-0.012}^{+0.012}$ & $0.914_{-0.013}^{+0.012}$ & $1.162_{-0.013}^{+0.0010}$ & $1.451_{-0.014}^{+0.020}$ & $2.085_{-0.074}^{+0.047}$ & $0.2676_{-0.0097}^{+0.0100}$ \\
\hline \multirow{7}{*}{$\begin{array}{l}d_{\odot} \\
(\mathrm{kpc}) \\
\mathrm{val} \times 10^{-3}\end{array}$} & Starless Cand. & 613 & $2.250_{-0.071}^{+0.075}$ & $3.395_{-0.056}^{+0.056}$ & $4.706_{-0.055}^{+0.057}$ & $6.52_{-0.29}^{+0.41}$ & $10.11_{-0.14}^{+0.15}$ & $1.424_{-0.072}^{+0.077}$ \\
\hline & Protostellar & 1027 & $2.160_{-0.056}^{+0.058}$ & $3.242_{-0.045}^{+0.046}$ & $4.788_{-0.054}^{+0.055}$ & $7.94_{-0.25}^{+0.23}$ & $11.11_{-0.17}^{+0.15}$ & $1.821_{-0.068}^{+0.070}$ \\
\hline & $70 \mu \mathrm{m}$ Unique & 311 & $2.18_{-0.13}^{+0.11}$ & $3.208_{-0.088}^{+0.088}$ & $4.680_{-0.089}^{+0.092}$ & $7.86_{-0.50}^{+0.45}$ & $10.90_{-0.25}^{+0.26}$ & $1.77_{-0.12}^{+0.13}$ \\
\hline & Mid IR & 504 & $2.086_{-0.060}^{+0.061}$ & $3.105_{-0.063}^{+0.060}$ & $4.623_{-0.079}^{+0.083}$ & $7.00_{-0.33}^{+0.39}$ & $10.70_{-0.24}^{+0.26}$ & $1.688_{-0.084}^{+0.090}$ \\
\hline & $\mathrm{H}_{2} \mathrm{O}$ & 323 & $2.315_{-0.098}^{+0.0907}$ & $3.437_{-0.080}^{+0.003}$ & $5.089_{-0.092}^{+0.091}$ & $8.31_{-0.31}^{+0.27}$ & $11.45_{-0.33}^{+0.22}$ & $1.99_{-0.12}^{+0.12}$ \\
\hline & $\mathrm{CH}_{3} \mathrm{OH}$ & 130 & $2.47_{-0.15}^{+0.15}$ & $4.07_{-0.18}^{+0.17}$ & $5.48_{-0.13}^{+0.14}$ & $9.16_{-0.21}^{+0.18}$ & $11.78_{-0.23}^{+0.21}$ & $2.41_{-0.20}^{+0.19}$ \\
\hline & $\mathrm{UCH}$ II & 101 & $2.85_{-0.27}^{+0.26}$ & $4.63_{-0.20}^{+0.19}$ & $6.41_{-0.47}^{+0.97}$ & $10.94_{-0.36}^{+0.33}$ & $13.03_{-0.23}^{+0.26}$ & $2.99_{-0.16}^{+0.16}$ \\
\hline \multirow{4}{*}{$\begin{array}{l}R_{\text {eq }}^{\text {Total }} \\
\text { (pc) }\end{array}$} & Starless Cand. & 613 & $0.352_{-0.010}^{+0.010}$ & $0.5286_{-0.0099}^{+0.010}$ & $0.842_{-0.016}^{+0.016}$ & $1.333_{-0.031}^{+0.035}$ & $2.152_{-0.064}^{+0.071}$ & $0.366_{-0.011}^{+0.012}$ \\
\hline & Protostellar & 1027 & $0.431_{-0.011}^{+0.011}$ & $0.705_{-0.012}^{+0.013}$ & $1.251_{-0.021}^{+0.021}$ & $2.162_{-0.043}^{+0.042}$ & $3.376_{-0.070}^{+0.075}$ & $0.638_{-0.016}^{+0.016}$ \\
\hline & $70 \mu \mathrm{m}$ Unique & 311 & $0.366_{-0.017}^{+0.018}$ & $0.591_{-0.018}^{+0.019}$ & $1.037_{-0.028}^{+0.031}$ & $1.706_{-0.046}^{+0.050}$ & $2.560_{-0.12}^{+0.091}$ & $0.512_{-0.021}^{+0.023}$ \\
\hline & Mid IR & 504 & $0.453_{-0.015}^{+0.016}$ & $0.758_{-0.018}^{+0.018}$ & $1.308_{-0.031}^{+0.031}$ & $2.257_{-0.067}^{+0.067}$ & $3.46_{-0.10}^{+0.11}$ & $0.648_{-0.023}^{+0.025}$ \\
\hline
\end{tabular}


Table 7

(Continued)

\begin{tabular}{|c|c|c|c|c|c|c|c|c|}
\hline Property & Indicator & $N$ & 10 & 25 & $50\left(\mu_{1 / 2}\right)$ & 75 & 90 & $\sigma_{1 / 2}$ \\
\hline & $\mathrm{H}_{2} \mathrm{O}$ & 323 & $0.579_{-0.023}^{+0.022}$ & $0.899_{-0.025}^{+0.027}$ & $1.574_{-0.041}^{+0.044}$ & $2.833_{-0.063}^{+0.068}$ & $3.981_{-0.093}^{+0.097}$ & $0.849_{-0.033}^{+0.033}$ \\
\hline & $\mathrm{CH}_{3} \mathrm{OH}$ & 130 & $0.812_{-0.054}^{+0.048}$ & $1.352_{-0.051}^{+0.056}$ & $2.309_{-0.10}^{+0.098}$ & $3.28_{-0.12}^{+0.13}$ & $4.56_{-0.19}^{+0.21}$ & $0.967_{-0.060}^{+0.064}$ \\
\hline & UCH II & 101 & $0.991_{-0.099}^{+0.14}$ & $1.653_{-0.088}^{+0.095}$ & $2.656_{-0.079}^{+0.073}$ & $3.876_{-0.11}^{+0.096}$ & $5.02_{-0.15}^{+0.15}$ & $1.111_{-0.066}^{+0.060}$ \\
\hline$R_{\mathrm{eq}}^{\mathrm{FWHM}}$ & Starless Cand. & 592 & $0.2593_{-0.0073}^{+0.0074}$ & $0.4005_{-0.0071}^{+0.0073}$ & $0.642_{-0.012}^{+0.012}$ & $0.964_{-0.019}^{+0.021}$ & $1.532_{-0.041}^{+0.044}$ & $0.2694_{-0.0082}^{+0.0083}$ \\
\hline \multirow[t]{6}{*}{ (pc) } & Protostellar & 1020 & $0.2264_{-0.0056}^{+0.0058}$ & $0.3653_{-0.0056}^{+0.0058}$ & $0.6166_{-0.0089}^{+0.0092}$ & $0.988_{-0.017}^{+0.017}$ & $1.509_{-0.032}^{+0.032}$ & $0.2847_{-0.0069}^{+0.0071}$ \\
\hline & $70 \mu \mathrm{m}$ Unique & 309 & $0.243_{-0.012}^{+0.0013}$ & $0.383_{-0.011}^{+0.0011}$ & $0.643_{-0.017}^{+0.015}$ & $0.996_{-0.030}^{+0.032}$ & $1.515_{-0.066}^{+0.052}$ & $0.286_{-0.012}^{+0.013}$ \\
\hline & Mid IR & 499 & $0.2247_{-0.0087}^{+0.0008}$ & $0.3543_{-0.0075}^{+0.00078}$ & $0.602_{-0.011}^{+0.011}$ & $0.981_{-0.026}^{+0.023}$ & $1.523_{-0.052}^{+0.050}$ & $0.2803_{-0.0091}^{+0.0095}$ \\
\hline & $\mathrm{H}_{2} \mathrm{O}$ & 323 & $0.2105_{-0.0095}^{+0.0010}$ & $0.3400_{-0.0089}^{+0.0006}$ & $0.551_{-0.012}^{+0.011}$ & $0.870_{-0.020}^{+0.020}$ & $1.326_{-0.043}^{+0.047}$ & $0.2499_{-0.0098}^{+0.00095}$ \\
\hline & $\mathrm{CH}_{3} \mathrm{OH}$ & 130 & $0.212_{-0.012}^{+0.012}$ & $0.318_{-0.012}^{+0.015}$ & $0.521_{-0.017}^{+0.018}$ & $0.837_{-0.031}^{+0.034}$ & $1.383_{-0.060}^{+0.054}$ & $0.241_{-0.012}^{+0.012}$ \\
\hline & UCH II & 101 & $0.243_{-0.017}^{+0.016}$ & $0.366_{-0.020}^{+0.023}$ & $0.577_{-0.021}^{+0.021}$ & $0.867_{-0.033}^{+0.042}$ & $1.434_{-0.073}^{+0.056}$ & $0.242_{-0.015}^{+0.015}$ \\
\hline \multirow{7}{*}{$\begin{array}{l}\Sigma_{\mathrm{H}_{2}}^{\text {Total }} \\
\left(\mathrm{g} \mathrm{cm}^{-2}\right) \\
\mathrm{val} \times 10^{3}\end{array}$} & Starless Cand. & 2222 & $5.67_{-0.17}^{+0.17}$ & $9.23_{-0.17}^{+0.17}$ & $14.53_{-0.20}^{+0.20}$ & $21.86_{-0.29}^{+0.30}$ & $30.88_{-0.47}^{+0.49}$ & $6.03_{-0.15}^{+0.14}$ \\
\hline & Protostellar & 2459 & $8.09_{-0.18}^{+0.18}$ & $12.56_{-0.18}^{+0.18}$ & $19.29_{-0.19}^{+0.19}$ & $28.10_{-0.27}^{+0.28}$ & $39.19_{-0.46}^{+0.47}$ & $7.50_{-0.14}^{+0.14}$ \\
\hline & $70 \mu \mathrm{m}$ Unique & 994 & $7.91_{-0.27}^{+0.18}$ & $11.72_{-0.25}^{+0.18}$ & $17.38_{-0.30}^{+0.31}$ & $24.80_{-0.42}^{+0.42}$ & $33.50_{-0.65}^{+0.46}$ & $6.32_{-0.21}^{+0.14}$ \\
\hline & Mid IR & 1022 & $8.09_{-0.30}^{+0.32}$ & $13.41_{-0.32}^{+0.32}$ & $21.26_{-0.31}^{+0.31}$ & $31.37_{-0.44}^{+0.46}$ & $44.19_{-0.71}^{+0.73}$ & $8.71_{-0.25}^{+0.25}$ \\
\hline & $\mathrm{H}_{2} \mathrm{O}$ & 556 & $11.27_{-0.36}^{+0.30}$ & $16.82_{-0.30}^{+0.29}$ & $23.68_{-0.32}^{+0.31}$ & $34.06_{-0.54}^{+0.54}$ & $48.7_{-1.1}^{+1.11}$ & $8.12_{-0.27}^{+0.27}$ \\
\hline & $\mathrm{CH}_{3} \mathrm{OH}$ & 298 & $10.18_{-0.48}^{+0.46}$ & $15.57_{-0.46}^{+0.40}$ & $21.47_{-0.37}^{+0.38}$ & $31.94_{-0.82}^{+0.54}$ & $48.8_{-1.5}^{+1.6}$ & $7.47_{-0.40}^{+0.41}$ \\
\hline & UCH II & 170 & $9.67_{-0.54}^{+0.55}$ & $14.71_{-0.56}^{+0.54}$ & $20.55_{-0.47}^{+0.48}$ & $30.41_{-0.87}^{+0.89}$ & $45.1_{-1.7}^{+1.8}$ & $7.33_{-0.44}^{+0.46}$ \\
\hline \multirow{7}{*}{$\begin{array}{l}\Sigma_{\mathrm{H}_{2}}^{\mathrm{FWHM}} \\
\left(\mathrm{g} \mathrm{cm}^{-2}\right) \\
\mathrm{val} \times 10^{3}\end{array}$} & Starless Cand. & 2138 & $5.54_{-0.21}^{+0.22}$ & $9.94_{-0.21}^{+0.21}$ & $16.56_{-0.26}^{+0.25}$ & $26.51_{-0.40}^{+0.41}$ & $42.00_{-0.86}^{+0.90}$ & $7.72_{-0.18}^{+0.19}$ \\
\hline & Protostellar & 2439 & $9.75_{-0.25}^{+0.24}$ & $16.53_{-0.28}^{+0.28}$ & $28.90_{-0.36}^{+0.37}$ & $51.61_{-0.59}^{+0.59}$ & $90.2_{-1.2}^{+1.2}$ & $15.13_{-0.27}^{+0.28}$ \\
\hline & $70 \mu \mathrm{m}$ Unique & 985 & $8.99_{-0.35}^{+0.33}$ & $14.23_{-0.35}^{+0.36}$ & $22.47_{-0.41}^{+0.44}$ & $34.76_{-0.66}^{+0.69}$ & $51.8_{-1.2}^{+1.2}$ & $9.57_{-0.32}^{+0.33}$ \\
\hline & Mid IR & 1010 & $10.01_{-0.43}^{+0.45}$ & $18.63_{-0.56}^{+0.57}$ & $35.22_{-0.66}^{+0.41}$ & $64.6_{-1.0}^{+1.00}$ & $112.0_{-2.2}^{+2.2}$ & $20.17_{-0.52}^{+0.52}$ \\
\hline & $\mathrm{H}_{2} \mathrm{O}$ & 556 & $19.61_{-0.81}^{+0.80}$ & $32.96_{-0.79}^{+0.79}$ & $56.27_{-0.91}^{+0.89}$ & $96.6_{-1.5}^{+1.5}$ & $166.8_{-3.7}^{+3.9}$ & $28.20_{-0.77}^{+0.76}$ \\
\hline & $\mathrm{CH}_{3} \mathrm{OH}$ & 297 & $17.4_{-1.2}^{+1.31}$ & $33.5_{-1.4}^{+1.4}$ & $64.3_{-1.5}^{+1.51}$ & $114.3_{-2.3}^{+2.3}$ & $196.8_{-5.7}^{+5.8}$ & $37.0_{-1.3}^{+1.2}$ \\
\hline & UCH II & 170 & $20.8_{-2.0}^{+2.1}$ & $40.5_{-1.7}^{+1.8}$ & $75.2_{-2.3}^{+2.3}$ & $123.6_{-2.8}^{+3.0}$ & $208.4_{-8.1}^{+9.1}$ & $39.7_{-1.7}^{+1.6}$ \\
\hline \multirow{7}{*}{$\begin{array}{l}\sum_{\mathrm{H}_{2}}^{\text {peak }} \\
\left(\mathrm{g} \mathrm{cm}^{-2}\right) \\
\mathrm{val} \times 10^{3}\end{array}$} & Starless Cand. & 2222 & $0.13_{-0.50}^{+0.49}$ & $8.66_{-0.39}^{+0.42}$ & $20.82_{-0.50}^{+0.51}$ & $38.45_{-0.76}^{+0.76}$ & $61.8_{-1.3}^{+1.3}$ & $14.05_{-0.35}^{+0.36}$ \\
\hline & Protostellar & 2459 & $7.72_{-0.53}^{+0.53}$ & $19.86_{-0.54}^{+0.53}$ & $40.75_{-0.70}^{+0.68}$ & $75.1_{-1.0}^{+1.1}$ & $125.3_{-1.8}^{+1.8}$ & $24.83_{-0.50}^{+0.52}$ \\
\hline & $70 \mu \mathrm{m}$ Unique & 994 & $4.76_{-0.87}^{+0.85}$ & $15.48_{-0.77}^{+0.73}$ & $30.83_{-0.87}^{+0.86}$ & $52.1_{-1.2}^{+1.3}$ & $78.8_{-2.1}^{+2.2}$ & $17.52_{-0.62}^{+0.63}$ \\
\hline & Mid IR & 1021 & $9.41_{-0.84}^{+0.81}$ & $23.09_{-0.94}^{+0.93}$ & $49.4_{-1.2}^{+1.21}$ & $92.7_{-1.8}^{+1.2}$ & $155.0_{-3.3}^{+3.2}$ & $31.24_{-0.88}^{+0.02}$ \\
\hline & $\mathrm{H}_{2} \mathrm{O}$ & 556 & $22.2_{-1.6}^{+1.6}$ & $43.7_{-1.5}^{+1.5}$ & $78.8_{-1.7}^{+1.6}$ & $133.4_{-2.5}^{+2.5}$ & $228.9_{-5.5}^{+5.7}$ & $41.1_{-1.4}^{+1.4}$ \\
\hline & $\mathrm{CH}_{3} \mathrm{OH}$ & 297 & $21.3_{-1.9}^{+2.1}$ & $45.1_{-2.2}^{+2.2}$ & $88.0_{-2.5}^{+2.5}$ & $155.7_{-3.7}^{+3.8}$ & $267.1_{-8.1}^{+8.4}$ & $50.6_{-2.0}^{+2.0}$ \\
\hline & $\mathrm{UCH}$ II & 170 & $27.2_{-2.9}^{+3.2}$ & $54.6_{-2.9}^{+2.9}$ & $101.2_{-3.5}^{+3.5}$ & $169.4_{-4.7}^{+4.9}$ & $279_{-12}^{+13}$ & $54.0_{-2.6}^{+2.8}$ \\
\hline \multirow{7}{*}{$\begin{array}{l}M_{\mathrm{H}_{2}}^{\text {Total }} \\
\left(M_{\odot}\right)\end{array}$} & Starless Cand. & 612 & $33.2_{-2.6}^{+2.8}$ & $86.1_{-4.6}^{+4.7}$ & $228_{-11}^{+11}$ & $639_{-33}^{+35}$ & $1610_{-100}^{+110}$ & $179.3_{-9.1}^{+9.5}$ \\
\hline & Protostellar & 1026 & $65.2_{-4.2}^{+4.3}$ & $183.8_{-7.8}^{+8.0}$ & $596_{-21}^{+22}$ & $1834_{-69}^{+73}$ & $4620_{-210}^{+210}$ & $503_{-18}^{+19}$ \\
\hline & $70 \mu \mathrm{m}$ Unique & 310 & $40.8_{-5.0}^{+5.6}$ & $122.1_{-9.4}^{+9.8}$ & $391_{-25}^{+26}$ & $1007_{-71}^{+78}$ & $2290_{-180}^{+200}$ & $318_{-21}^{+22}$ \\
\hline & Mid IR & 504 & $83.6_{-6.7}^{+7.0}$ & $215_{-13}^{+14}$ & $699_{-36}^{+37}$ & $2140_{-110}^{+110}$ & $5060_{-320}^{+330}$ & $587_{-31}^{+31}$ \\
\hline & $\mathrm{H}_{2} \mathrm{O}$ & 323 & $140_{-13}^{+14}$ & $384_{-25}^{+26}$ & $1164_{-66}^{+70}$ & $3350_{-200}^{+220}$ & $7500_{-420}^{+440}$ & $961_{-56}^{+60}$ \\
\hline & $\mathrm{CH}_{3} \mathrm{OH}$ & 130 & $224_{-45}^{+61}$ & $700_{-62}^{+75}$ & $1970_{-180}^{+180}$ & $5180_{-310}^{+310}$ & $9930_{-800}^{+980}$ & $1570_{-140}^{+150}$ \\
\hline & UCH II & 101 & $354_{-76}^{+80}$ & $970_{-110}^{+110}$ & $2590_{-180}^{+200}$ & $5520_{-350}^{+350}$ & $9810_{-860}^{+920}$ & $1930_{-140}^{+150}$ \\
\hline \multirow{7}{*}{$\begin{array}{l}M_{\mathrm{H}_{2}}^{\mathrm{FWM}} \\
\left(M_{\odot}\right)\end{array}$} & Starless Cand. & 613 & $19.7_{-2.0}^{+2.0}$ & $61.5_{-3.6}^{+3.5}$ & $166.2_{-7.5}^{+8.1}$ & $450_{-22}^{+25}$ & $1141_{-72}^{+79}$ & $133.1_{-6.3}^{+6.7}$ \\
\hline & Protostellar & 1026 & $43.2_{-2.9}^{+2.9}$ & $119.7_{-4.9}^{+5.2}$ & $341_{-11}^{+12}$ & $944_{-36}^{+35}$ & $2068_{-83}^{+91}$ & $274.5_{-9.6}^{+10}$ \\
\hline & $70 \mu \mathrm{m}$ Unique & 310 & $29.9_{-3.7}^{+4.3}$ & $86.6_{-6.6}^{+6.8}$ & $257_{-15}^{+16}$ & $641_{-43}^{+40}$ & $1370_{-110}^{+120}$ & $203_{-13}^{+14}$ \\
\hline & Mid IR & 503 & $53.0_{-4.2}^{+4.4}$ & $133.7_{-7.1}^{+7.4}$ & $376_{-17}^{+18}$ & $1037_{-50}^{+54}$ & $2310_{-130}^{+140}$ & $299_{-15}^{+16}$ \\
\hline & $\mathrm{H}_{2} \mathrm{O}$ & 323 & $82.9_{-8.3}^{+8.8}$ & $198_{-11}^{+12}$ & $494_{-27}^{+30}$ & $1373_{-74}^{+75}$ & $2710_{-160}^{+170}$ & $378_{-22}^{+24}$ \\
\hline & $\mathrm{CH}_{3} \mathrm{OH}$ & 130 & $109_{-19}^{+20}$ & $294_{-26}^{+28}$ & $717_{-61}^{+70}$ & $1770_{-98}^{+110}$ & $3230_{-240}^{+320}$ & $546_{-49}^{+55}$ \\
\hline & UCH II & 101 & $152_{-31}^{+34}$ & $394_{-39}^{+38}$ & $1010_{-71}^{+74}$ & $1850_{-100}^{+110}$ & $3180_{-420}^{+450}$ & $689_{-48}^{+49}$ \\
\hline \multirow{7}{*}{$\begin{array}{l}n_{\mathrm{c}} \\
\left(\mathrm{cm}^{-3}\right)\end{array}$} & Starless Cand. & 591 & $287_{-31}^{+30}$ & $645_{-36}^{+36}$ & $1338_{-57}^{+61}$ & $2800_{-140}^{+160}$ & $6560_{-580}^{+690}$ & $863_{-45}^{+47}$ \\
\hline & Protostellar & 1019 & $390_{-24}^{+25}$ & $848_{-30}^{+32}$ & $1857_{-54}^{+57}$ & $4160_{-140}^{+150}$ & $9880_{-580}^{+640}$ & $1253_{-45}^{+46}$ \\
\hline & $70 \mu \mathrm{m}$ Unique & 308 & $322_{-43}^{+43}$ & $721_{-51}^{+53}$ & $1475_{-80}^{+84}$ & $3000_{-190}^{+220}$ & $6370_{-660}^{+860}$ & $940_{-66}^{+64}$ \\
\hline & Mid IR & 498 & $415_{-36}^{+36}$ & $917_{-48}^{+49}$ & $2120_{-91}^{+93}$ & $4760_{-220}^{+230}$ & $11580_{-900}^{+1000}$ & $1468_{-72}^{+73}$ \\
\hline & $\mathrm{H}_{2} \mathrm{O}$ & 323 & $635_{-48}^{+50}$ & $1290_{-75}^{+75}$ & $2780_{-140}^{+140}$ & $6560_{-340}^{+320}$ & $15670_{-880}^{+1000}$ & $1890_{-110}^{+110}$ \\
\hline & $\mathrm{CH}_{3} \mathrm{OH}$ & 130 & $686_{-87}^{+89}$ & $1550_{-120}^{+110}$ & $3080_{-210}^{+220}$ & $8090_{-520}^{+550}$ & $24900_{-1700}^{+1700}$ & $2060_{-160}^{+180}$ \\
\hline & UCH II & 101 & $539_{-66}^{+73}$ & $1300_{-140}^{+180}$ & $3460_{-240}^{+260}$ & $7260_{-420}^{+440}$ & $15600_{-1400}^{+1600}$ & $2540_{-190}^{+200}$ \\
\hline
\end{tabular}


Table 7

(Continued)

\begin{tabular}{|c|c|c|c|c|c|c|c|c|}
\hline Property & Indicator & $N$ & 10 & 25 & $50\left(\mu_{1 / 2}\right)$ & 75 & 90 & $\sigma_{1 / 2}$ \\
\hline$t_{\mathrm{ff}, \mathrm{c}}$ & Starless Cand. & 590 & $0.379_{-0.018}^{+0.018}$ & $0.581_{-0.016}^{+0.016}$ & $0.839_{-0.018}^{+0.019}$ & $1.207_{-0.031}^{+0.034}$ & $1.795_{-0.085}^{+0.099}$ & $0.299_{-0.014}^{+0.014}$ \\
\hline (year) & Protostellar & 1018 & $0.3092_{-0.0094}^{+0.0093}$ & $0.4763_{-0.0083}^{+0.0086}$ & $0.713_{-0.011}^{+0.011}$ & $1.053_{-0.019}^{+0.019}$ & $1.545_{-0.047}^{+0.050}$ & $0.2729_{-0.0083}^{+0.0084}$ \\
\hline $\mathrm{val} \times 10^{-6}$ & $70 \mu \mathrm{m}$ Unique & 307 & $0.385_{-0.024}^{+0.021}$ & $0.560_{-0.019}^{+0.019}$ & $0.799_{-0.022}^{+0.022}$ & $1.139_{-0.040}^{+0.043}$ & $1.682_{-0.099}^{+0.12}$ & $0.275_{-0.017}^{+0.018}$ \\
\hline & Mid IR & 498 & $0.286_{-0.011}^{+0.012}$ & $0.446_{-0.011}^{+0.011}$ & $0.667_{-0.014}^{+0.014}$ & $1.013_{-0.026}^{+0.028}$ & $1.503_{-0.062}^{+0.070}$ & $0.264_{-0.011}^{+0.011}$ \\
\hline & $\mathrm{H}_{2} \mathrm{O}$ & 322 & $0.2456_{-0.0078}^{+0.0071}$ & $0.379_{-0.010}^{+0.010}$ & $0.583_{-0.014}^{+0.015}$ & $0.857_{-0.024}^{+0.025}$ & $1.218_{-0.044}^{+0.051}$ & $0.229_{-0.010}^{+0.010}$ \\
\hline & $\mathrm{CH}_{3} \mathrm{OH}$ & 129 & $0.1948_{-0.0064}^{+0.0072}$ & $0.341_{-0.012}^{+0.011}$ & $0.553_{-0.019}^{+0.021}$ & $0.782_{-0.028}^{+0.032}$ & $1.173_{-0.070}^{+0.080}$ & $0.221_{-0.014}^{+0.015}$ \\
\hline$\alpha_{\text {vir }}$ & Starless Cand. & 174 & $0.173_{-0.024}^{+0.024}$ & $0.339_{-0.028}^{+0.030}$ & $0.726_{-0.058}^{+0.063}$ & $1.90_{-0.19}^{+0.23}$ & $5.74_{-0.89}^{+1.1}$ & $0.498_{-0.049}^{+0.056}$ \\
\hline & Protostellar & 637 & $0.167_{-0.012}^{+0.012}$ & $0.338_{-0.015}^{+0.015}$ & $0.676_{-0.025}^{+0.026}$ & $1.353_{-0.058}^{+0.059}$ & $2.90_{-0.21}^{+0.24}$ & $0.415_{-0.020}^{+0.021}$ \\
\hline & $70 \mu \mathrm{m}$ Unique & 153 & $0.167_{-0.025}^{+0.026}$ & $0.344_{-0.032}^{+0.032}$ & $0.664_{-0.049}^{+0.052}$ & $1.32_{-0.12}^{+0.13}$ & $2.85_{-0.40}^{+0.49}$ & $0.400_{-0.039}^{+0.042}$ \\
\hline & Mid IR & 319 & $0.153_{-0.015}^{+0.016}$ & $0.302_{-0.018}^{+0.018}$ & $0.591_{-0.030}^{+0.032}$ & $1.162_{-0.063}^{+0.067}$ & $2.18_{-0.18}^{+0.22}$ & $0.359_{-0.024}^{+0.024}$ \\
\hline & $\mathrm{H}_{2} \mathrm{O}$ & 276 & $0.204_{-0.022}^{+0.023}$ & $0.407_{-0.026}^{+0.026}$ & $0.810_{-0.042}^{+0.044}$ & $1.564_{-0.098}^{+0.10}$ & $3.33_{-0.34}^{+0.39}$ & $0.484_{-0.033}^{+0.035}$ \\
\hline & $\mathrm{CH}_{3} \mathrm{OH}$ & 94 & $0.204_{-0.036}^{+0.043}$ & $0.430_{-0.045}^{+0.047}$ & $0.866_{-0.072}^{+0.074}$ & $1.61_{-0.15}^{+0.18}$ & $3.29_{-0.48}^{+0.61}$ & $0.513_{-0.056}^{+0.061}$ \\
\hline
\end{tabular}

Note. Columns list the (1) physical property, unit, and numerical scale factor if applied, (2) star formation indicator category, (3) number of clumps in calculation, (4-8) 10th through 90th percentile, and (9) median absolute deviation. Uncertainties show the $1 \sigma$ confidence interval.

associated $\mathrm{CH}_{3} \mathrm{OH}$ masers and/or $\mathrm{UCH}$ II regions show a relative deficit of distances from 2 to $4 \mathrm{kpc}$. This could be an effect from the method of resolving distances with the $8 \mu \mathrm{m}$ absorption prior, where better spatially resolved clumps at the near distance are dominated by mid-IR emission from a high-mass YSO. These regions may not have the necessary contrast in the GLIMPSE $8 \mu \mathrm{m}$ maps to result in a wellconstrained distance estimate, whereas further, and thus larger, clumps could have sufficient surrounding $8 \mu \mathrm{m}$ absorption. In addition, the PPV method of associating nearby clumps with known distances for a fixed radius in angle and a fixed velocity better associates regions at larger distances where the angular separation between clumps is smaller.

When comparing distance-independent quantities between starless candidate and protostellar categories, the total number of sources is nearly equal (2223 SCCs versus 2460 protostarcontaining clumps); however, when comparing distancedependent physical properties, it should be noted that in the distance sample there are 1.675 times as many protostellarcontaining clumps than SCCs (613 SCCs versus 1027 protostellar clumps). The fraction of clumps in the distance sample that contain at least one star formation indicator is shown as a function of heliocentric distance in Figure 9. The median protostellar clump fraction in the distance sample is 0.60 , and there is no significant trend in this ratio out to heliocentric distances of $12 \mathrm{kpc}$. Since only 58 clumps (3.5\%) in this study have maximum-likelihood distances greater than $12 \mathrm{kpc}$, this result indicates that differences in the properties of starless candidates compared to protostellar clumps are not driven by a bias in the protostellar fraction by distance.

We can only calculate distance-dependent quantities from the subset of clumps in each category of the distance sample. We run MC simulations for both the subsamples that have a well-constrained DPDF and those without to test how this selection criterion may bias our distributions. Figure 8(a) shows the observed total flux density $S_{1.1}^{\text {Total }}$ for each indicator, with the total sample (light gray) and distance sample (dark gray). Figure 8(b) shows the cumulative results of $10^{4}$ draws from the MC simulation of the $1.1 \mathrm{~mm}$ flux density Gaussian deviates and R08 AGB contamination resampling.
The subsamples with DPDFs have greater median $S_{1.1}$ when compared to full samples. Similarly, Figure $8(d)$ shows the distribution of the ratio of medians for each MC simulation. All indicator categories show a factor of 1.5-2.2 higher median flux for clumps with DPDFs; however, there is not a consistent trend in the ratio between star formation indicators. The ratio of median flux densities for protostar-containing clumps is only a factor of 1.1-1.2 times larger than the corresponding ratio for SCCs. If we make the simple assumption that sources without well-constrained DPDFs follow the average DPDF distributions for the distance sample, then this result implies that physical quantities that depend linearly on flux density (i.e., mass) are biased by factors of 1.5-2.2 larger in the distance sample versus the total sample.

\subsection{Flux Density, Flux Concentration, and Size}

The observed $1.1 \mathrm{~mm}$ flux densities increase toward more extreme indicators from SCCs to UCH II associated clumps (Figure 8(a)). The median total $1.1 \mathrm{~mm}$ flux density of starless clump candidates is $0.356 \pm 0.005 \mathrm{Jy}$ and is a factor of 15 lower in total flux density compared to the median for UCH II associated clumps. The starless candidate and UCH II associated clump distributions are distinct where $99 \%$ of starless candidates have lower total $1.1 \mathrm{~mm}$ flux density than the median total flux of UCH II associated clumps. Even the presence of a deeply embedded YSO observed as a compact Hi-GAL $70 \mu \mathrm{m}$ source preferentially increases the median observed flux density by a factor of $\sim 1.8$. Figure 9 shows these trends in an alternative way as a strongly increasing protostellar clump fraction with increasing $1.1 \mathrm{~mm}$ flux density. The ratio crosses 0.5 around $500 \mathrm{mJy}$ and approaches 1.0 above $5 \mathrm{Jy}$. Without internal star formation activity, starless clumps would only be externally heated by the interstellar radiation field (ISRF; Evans et al. 2002; Jørgensen et al. 2006; Wilcock et al. 2012). As a result, clumps with weaker internal heating will have colder dust temperatures and lower observed $1.1 \mathrm{~mm}$ flux densities (see Section 5.3).

The deconvolved clump equivalent angular radius $\theta_{\mathrm{eq}}$ is calculated by subtracting the BGPS beam $\theta_{\mathrm{HPBW}}=33^{\prime \prime}$ 

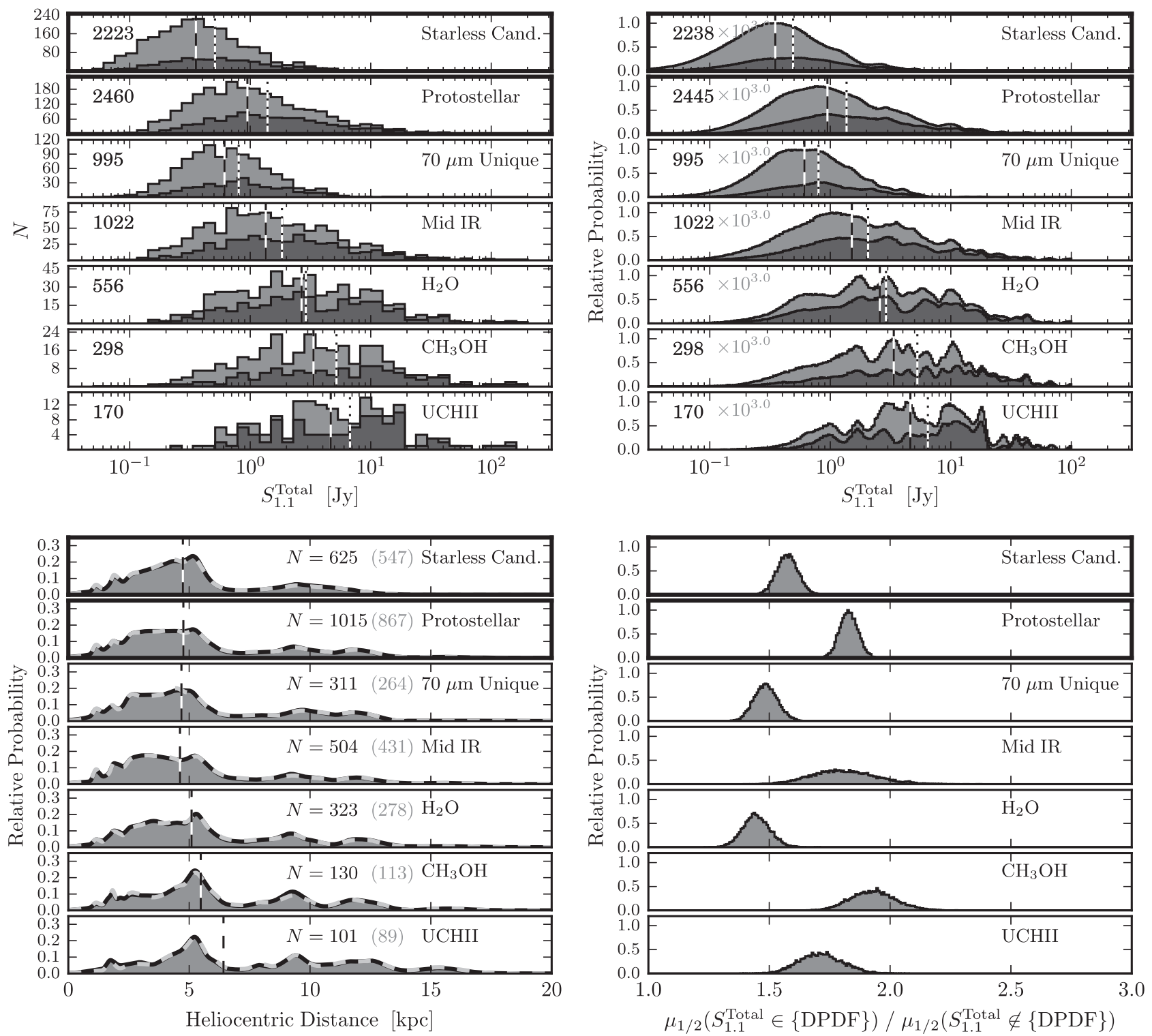

Figure 8. Distributions of total flux density for clumps in the full sample (light gray) and distance sample (dark gray) by star formation indicator. Top left: flux density histogram of observed values. Top right: flux density distribution drawn from MC simulation. Bottom left: average DPDF for the expanded DPDF sample (black line) and original DPDF sample (dashed gray line). The number $N$ of the clumps is shown in black and gray, respectively. Bottom right: ratio of median total flux density of clumps with a DPDF to clumps without a DPDF. Clumps in the distance sample have a factor of $\sim 1.5-2.0$ higher median flux density than clumps not in the distance sample. The star formation indicator is shown in the upper right of each panel.

$\left(\Omega_{\text {beam }}=1234 \operatorname{arcsec}^{2}\right)$ in quadrature from the observed equivalent angular radius $\theta_{\mathrm{obs}}=\Omega_{\mathrm{obs}} / \pi$ calculated directly from the sum of the 7 !' $2 \times 7$ !! 2 map pixels in the Bolocat label-mask frames

$$
\theta_{\mathrm{eq}}=\sqrt{\frac{\Omega_{\mathrm{obs}}}{\pi}-\frac{\Omega_{\mathrm{beam}}}{\pi}},
$$

where $\Omega_{\mathrm{obs}}$ is either the total solid angle defined by the Bolocat v2.0 seeded watershed algorithm or the FWHM solid angle defined by the contour at half-maximum flux density. Quantities that are derived from the Bolocat label masks of the BGPS maps are sensitive to the spatial filtering imposed by the principal component analysis performed on the Bolocam time-stream data. A clump size based on the FWHM definition is more robust to spatial filtering and is also more prevalent in the literature (e.g., Beuther et al. 2002; Shirley et al. 2003). In the following analyses, we shall use both total size definition, i.e., the full label masks, and the FWHM size definition to calculate derived properties.

Figure 10 (a) shows $\Omega^{\text {Total }}$ for each indicator, with an increasing trend in the distributions from median $3.62 \times 10^{3} \operatorname{arcsec}^{2}\left(1.01 \operatorname{arcmin}^{2}\right)$ for the starless candidates to $1.81 \times 10^{4} \operatorname{arcsec}^{2}\left(5.03 \operatorname{arcmin}^{2}\right)$ for the UCH II associated clumps. There is also a systematically increasing trend in the concentration of the $1.1 \mathrm{~mm}$ flux density distributions when sorted by indicator. Figure 10(b) shows the ratio in deconvolved solid angles, or total to FWHM "concentration," defined as $\Omega^{\text {Total }} / \Omega^{\text {FWHM }}$. Clumps that are near uniform brightness and with a peak brightness less than twice the $1 \sigma$ ( $\sim 0.1-0.5 \mathrm{Jy})$ cutoff threshold of the clump boundaries should have concentrations near unity, while clumps with highly concentrated brightness distributions will have large concentration 

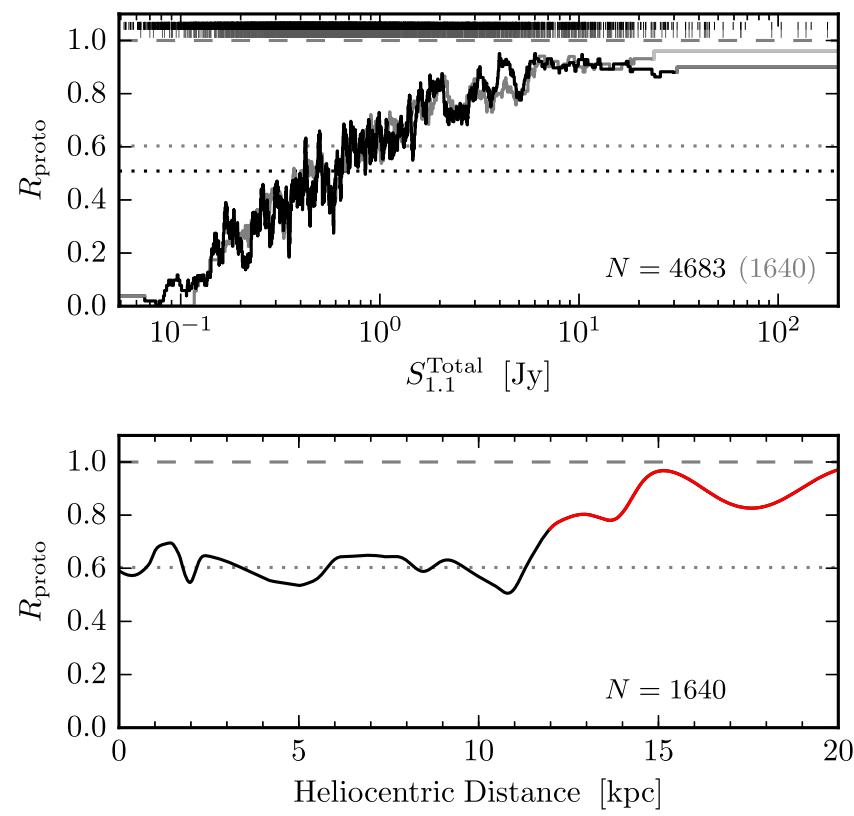

Figure 9. Protostellar fraction distributions, computed using the MC simulations. Top: total flux density. The black line shows $R_{\text {proto }}$ for a moving window of 50 sources over the full overlap sample, and the distance sample is shown in gray. Vertical lines show the clump $S_{1.1}^{\text {Total }}$ values. Bottom: heliocentric distance. The solid line shows $R_{\text {proto }}$ computed as a weighted sum of the DPDFs. $R_{\text {proto }}$ is approximately constant at $<12 \mathrm{kpc}$, suggesting that the star formation indicators are mostly complete for BGPS clumps. In red are the 58 clumps with $d_{\mathrm{ML}}>12 \mathrm{kpc}$ that compose $3.5 \%$ of the distance sample. Sample protostellar fractions are shown by horizontal dotted lines.

values $(\gg 1)$. A strong increasing trend is seen from a median ratio $\Omega^{\text {Total }} / \Omega^{\mathrm{FWHM}}$ for the starless candidate phase at 1.55-16.38 for UCH II clumps. Clumps associated with $\mathrm{H}_{2} \mathrm{O}$ masers, $\mathrm{CH}_{3} \mathrm{OH}$ masers, and $\mathrm{UCH}$ II regions are concentrated at similar levels and also more concentrated than those with less extreme indicators.

The equivalent physical radius $R_{\mathrm{eq}}$ is calculated from the observed $\Omega$ when projected to a heliocentric distance $d_{\odot}$ drawn from the DPDFs

$$
R_{\mathrm{eq}}=0.29\left(\frac{\theta_{\mathrm{eq}}}{\operatorname{arcmin}}\right)\left(\frac{d_{\odot}}{\mathrm{kpc}}\right) .
$$

Figure 11 shows the equivalent radius distributions for the total (Figure 11(a)) and FWHM (Figure 11(b)) definitions. The median $R_{\mathrm{eq}}^{\text {Total }}$ increases from $0.842 \pm 0.017$ to $2.66 \pm 0.08 \mathrm{pc}$ from starless candidates to $\mathrm{UCH}$ II clumps; however, little separation among different indicators is observed using the FWHM definition with median $R_{\mathrm{eq}}^{\mathrm{FWHM}} \sim 0.6 \mathrm{pc}$. The $R_{\mathrm{eq}}^{\text {Total }}$ in this study are larger than those found by Schlingman et al. (2011) (median $R_{\text {eq }}=0.75$ ) for a subset of known 529 sources taken from the BGPS v1.0. This discrepancy can be attributed to the recovery of flux at lower spatial frequencies in the BGPS v2.0 maps, resulting in larger clumps (Ginsburg et al. 2013). The different trends observed between the total source size and FWHM size are more striking and can be explained by offsetting factors. Clumps containing indicators of more luminous protostars (i.e., $\mathrm{CH}_{3} \mathrm{OH}$ masers, $\mathrm{UCH}$ II) have the highest flux concentrations, which partially offset their larger total solid angle, resulting in their FWHM sizes remaining nearly the same on median as SCCs and clumps with lower-luminosity protostellar indicators. This result highlights the need to explore multiple size definitions in the analysis of source physical properties.

\subsection{Gas Kinetic Temperature and Line Width}

$\mathrm{NH}_{3}$ is an excellent intermediate to dense gas tracer with an effective excitation of $\sim 10^{3} \mathrm{~cm}^{-3}$ for the lowest pure inversion transition (Shirley 2015). Fitting the intensities of the lowestenergy inversion transitions provides estimates of the gas kinetic temperature (Ho \& Townes 1983). The rotation temperature of the $(1,1)$ and $(2,2) \mathrm{p}-\mathrm{NH}_{3}$ inversion transitions saturates for $T_{\mathrm{K}}>30 \mathrm{~K}$, but the simultaneously observed $(3,3)$ transitions retain sensitivity to $T_{\mathrm{K}}<100 \mathrm{~K}$ if the $\mathrm{o}-\mathrm{NH}_{3}(3,3)$ transition is not masing (Danby et al. 1988). Figure 12 shows the distributions of $\mathrm{NH}_{3}$-derived gas kinetic temperature with values ranging between $T_{\mathrm{K}} \sim 10$ and $100 \mathrm{~K}$. The starless candidate phase has a median $T_{\mathrm{K}}=13.96 \pm 0.10 \mathrm{~K}$ with an increasing trend to $T_{\mathrm{K}}=27.2 \pm 0.2 \mathrm{~K}$ for $\mathrm{UCH}$ II clumps. The clumps uniquely associated with compact Hi-GAL $70 \mu \mathrm{m}$ sources, a sign of deeply embedded protostellar activity, show only a slight enhancement in the gas kinetic temperature. As a clump evolves from a quiescent phase to one with active star formation, radiative heating from star formation is expected to raise the gas kinetic temperature. With more extreme indicators of star formation activity suggesting higher-luminosity YSOs, the $\mathrm{CH}_{3} \mathrm{OH}$ and $\mathrm{UCH}_{\text {II }}$ regions containing clumps are subject to stronger radiative heating from massive star formation. The SCC distribution does display a positive skew. This could be a result of undetected embedded sources or enhancement by the local radiation field from neighboring starforming regions. The lower $S_{1.1}$ in the candidate starless clump stage shown in Figure $8 \mathrm{can}$, in part, be attributed to this observed trend in $T_{\mathrm{K}}$. The flux density of a modified blackbody is 4.9 times higher at $T_{\mathrm{K}}=30 \mathrm{~K}$ than $10 \mathrm{~K}$ at $1.1 \mathrm{~mm}$. We shall account for the temperature differences observed among the different star formation indicators in our calculations of the clump mass in Section 5.4.

The observed FWHM line widths of $\mathrm{NH}_{3}$ for clumps may be broadened as a result of a variety of factors, including thermal broadening, bulk motion, optical depth, and turbulence. In the following analysis we use the $\mathrm{NH}_{3}$ rather than $\mathrm{HCO}^{+}$line width because the $\mathrm{NH}_{3}$ observations have three times better spectral resolution, $0.3 \mathrm{~km} \mathrm{~s}^{-1}$, and $\mathrm{NH}_{3}$ has lower optical depths, $\tau_{\mathrm{NH}_{3}(1,1)} \sim 1-5$ compared to $\tau_{\mathrm{HCO}^{+}(3-2)} \sim 10$ (see Shirley et al. 2013). Figures 12(b) shows the distributions of observed $\mathrm{NH}_{3}$ line width, indicating a factor of two increase from $1.339 \pm 0.014 \mathrm{~km} \mathrm{~s}^{-1}$ on median toward starless candidates to $2.736 \pm 0.03 \mathrm{~km} \mathrm{~s}^{-1}$ on median toward $\mathrm{UCH}$ II associated clumps. For gas temperatures between 10 and $30 \mathrm{~K}$ the thermal line width is $\Delta v_{\text {therm }}\left(\mathrm{NH}_{3}\right)=0.16-0.28 \mathrm{~km} \mathrm{~s}^{-1}$. The observed $\mathrm{NH}_{3}$ line widths at $>1 \mathrm{~km} \mathrm{~s}^{-1}$ suggest that supersonic turbulence exists on scales smaller than the $33^{\prime \prime}$ beam of the BGPS. The quiescent starless candidate phase and the deeply embedded candidate phase of uniquely identified Hi-GAL $70 \mu \mathrm{m}$ compact sources have $\Delta v_{\text {turb }} /$ $\Delta v_{\text {therm }} \sim 10$. The observed increase in line width with more extreme star formation indicators could be due to an increase in turbulent feedback in more luminous protostellar clumps. More luminous protostars will drive more powerful jets and outflows (see Bontemps et al. 1996), as well as provide more radiative feedback on the surrounding clump that can drive turbulence (see Krumholz \& Thompson 2012). Alternatively, the larger 

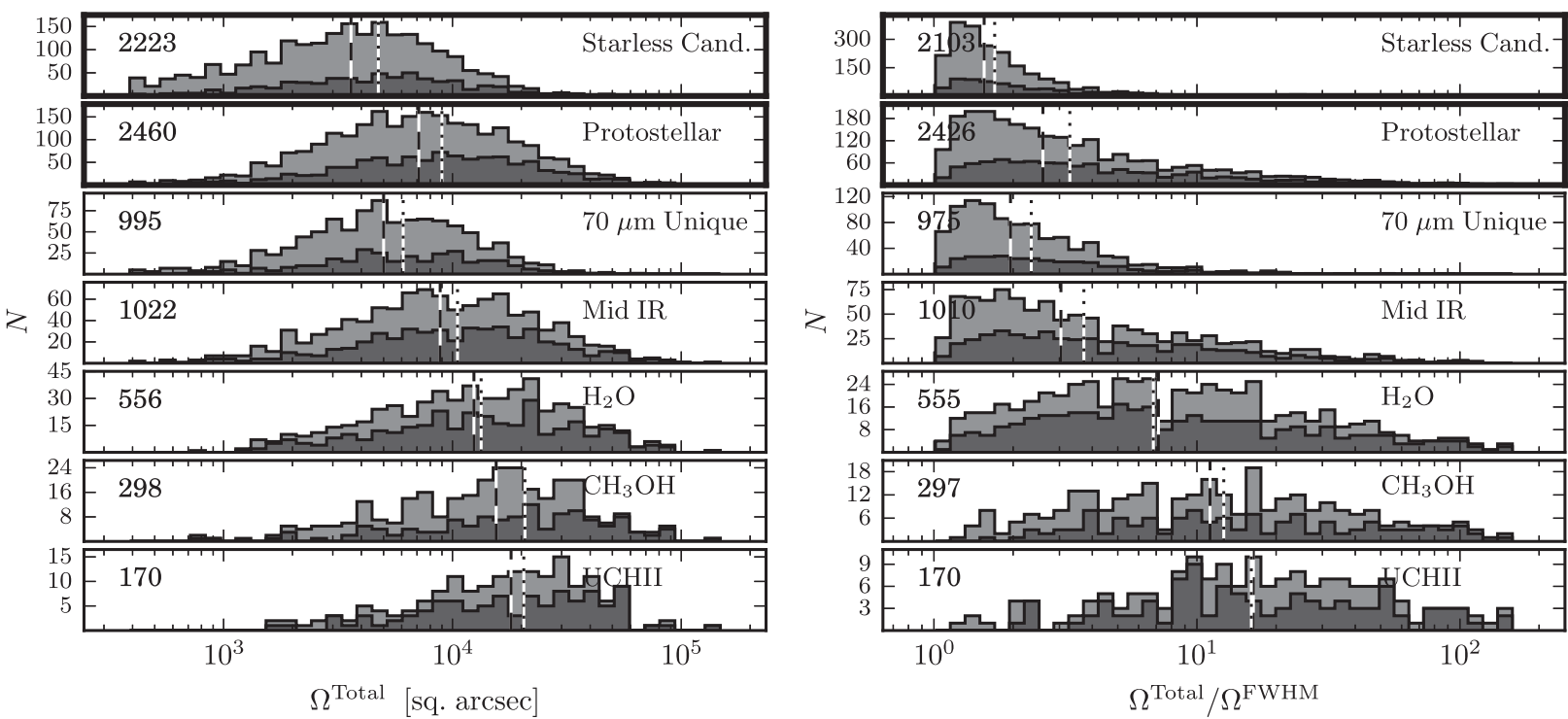

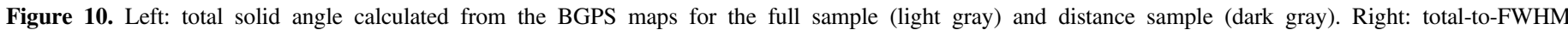

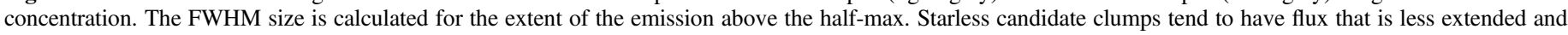
less concentrated than more extreme indicators.

line widths toward clumps with more extreme indicators could be due to larger bulk-flow motions that are unresolved at the GBT angular resolution or a systematic tendency for highermass stars to form in more turbulent regions. Without higherresolution observations of the clumps, it is difficult to determine which scenario drives the observed trend.

Prior observations of a significantly smaller subsample of BGPS clumps have indicated a breakdown in the line widthsize relationship (Schlingman et al. 2011). We plot the line width-size relationship for SCCs and protostellar clumps observed in $\mathrm{NH}_{3}$, shown in Figure 13. The Spearman's rank correlation coefficients for SCCs and protostellar clumps are $\rho_{\mathrm{sp}}=0.24$ and $\rho_{\mathrm{sp}}=0.50$, respectively, from the MC simulations. The starless clumps appear uncorrelated, while protostellar clumps have a weak correlation that is only marginally better than the Schlingman et al. (2011) correlation $\left(\rho_{\mathrm{sp}}=0.40\right)$. SCCs, as traced by $\mathrm{NH}_{3}$ emission, seem to have decoupled from the supersonic scaling relationship observed toward molecular clouds more so than protostellar clumps. Indeed, the observed $\mathrm{NH}_{3}$ model-fit velocity dispersion (expressed as FWHM line width) is below the extrapolated relationship observed by Larson (1981) for molecular clouds traced by $\mathrm{CO} 1-0$. While these clumps themselves are still turbulent structures, their level of turbulence appears dissipated compared to the expected relationship in clouds when observed on clump spatial scales.

\subsection{Mass Calculations}

\subsubsection{Mass Surface Density}

The mean mass surface density is calculated using

$$
\Sigma_{\mathrm{H}_{2}}=\frac{S_{1.1}}{B_{1.1}\left(T_{\text {dust }}\right) \kappa_{1.1} \zeta} \frac{1}{\Omega} \text {, }
$$

where $B_{1.1}$ is the Planck function, $\kappa_{1.1}$ is the dust opacity, $\zeta$ is the dust-to-gas ratio, here assumed to be $1 / 100, \Omega$ is the source solid angle, and $S_{1.1}$ is the source integrated flux density. Both the total and FWHM size definitions are used for $\Omega$ or $S_{1.1}$. We assume that the $1.1 \mathrm{~mm}$ emission is optically thin and use Gaussian deviates to sample the statistical uncertainty. We also assume $\mathrm{OH} 5$ dust opacities calculated for coagulated grains with thin ice mantles (Ossenkopf \& Henning 1994). For the MC calculation, we draw Gaussian deviates from the observational uncertainty on $T_{\mathrm{K}}$ and assume that the dust temperature is equal to the gas kinetic temperature. Because 47\% (772/ 1640) of clumps with a distance lack $T_{\mathrm{K}}$ measurements, for those clumps we draw $T_{\mathrm{K}}$ from that indicator's observed distribution of $T_{\mathrm{K}}$ (Figure 12(a)). This takes into account the trend of increasing gas $T_{\mathrm{K}}$ for clumps associated with more extreme star formation indicators. For clumps associated with multiple star formation indicators, the indicator with the hottest median $T_{\mathrm{K}}$ is used.

Figure 14 shows $\Sigma_{\mathrm{H}_{2}}^{\text {Total }}$ with an increasing trend toward higher $\Sigma_{\mathrm{H}_{2}}^{\text {Total }}$ for clumps associated with more extreme star formation indicators. The median $\Sigma_{\mathrm{H}_{2}}^{\text {Total }}$ increases from median values $\quad \Sigma_{\mathrm{H}_{2}}^{\text {Total }}=0.0145 \pm 0.0002 \mathrm{~g} \mathrm{~cm}^{-2}$ and $\Sigma_{\mathrm{H}_{2}}^{\mathrm{FWHM}}=$ $0.0165 \pm 0.0003 \mathrm{~g} \mathrm{~cm}^{-2}$ for the starless candidate clumps to $\Sigma_{\mathrm{H}_{2}}^{\text {Total }}=0.0207 \pm 0.0005 \mathrm{~g} \mathrm{~cm}^{-2} \quad$ and $\quad \Sigma_{\mathrm{H}_{2}}^{\mathrm{FWHM}}=0.076 \pm$ $0.002 \mathrm{~g} \mathrm{~cm}^{-2}$ for the $\mathrm{UCH}$ II associated clumps. A similar trend in increasing column density from quiescent (potentially starless) clumps to protstellar clumps has also been observed by other (sub)millimeter Galactic plane surveys (see Csengeri et al. 2014; Guzmán et al. 2015).

The factor of four increase in $\Sigma_{\mathrm{H}_{2}}^{\mathrm{FWHM}}$ is consistent with the greater flux densities and flux concentrations observed toward clumps with more extreme indicators. The median mass surface density for protostellar sources is close to the purported threshold for star formation of approximately $120 M_{\odot} \mathrm{pc}^{-2}$ (or $0.025 \mathrm{~g} \mathrm{~cm}^{-2}$; Heiderman et al. 2010; Lada et al. 2010). Since half of these clumps presumably contain protostellar sources despite being below this threshold, this result highlights that BGPS clumps themselves contain significant substructures that are at higher mass surface densities. 

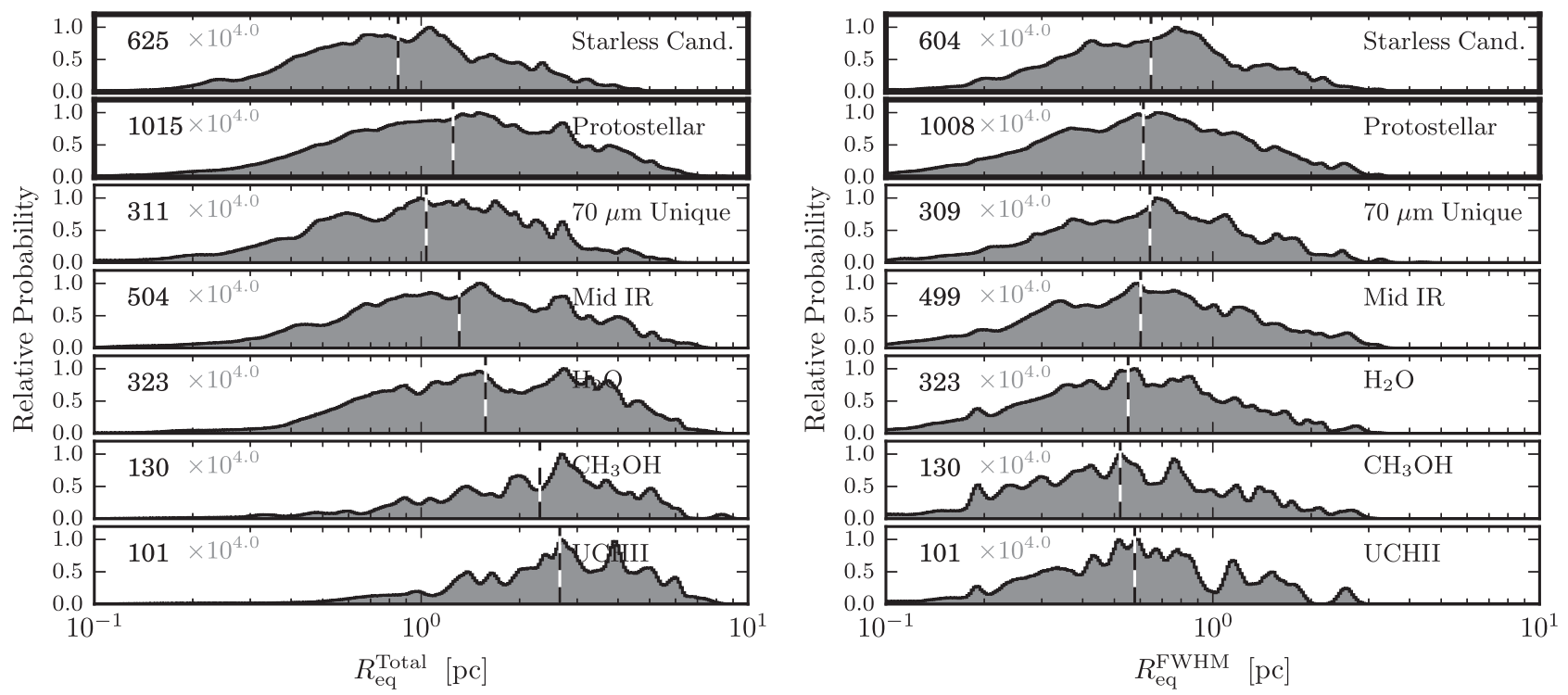

Figure 11. Physical equivalent radius histograms from the MC simulations for each star formation indicator sample. Left: physical total radius. Right: physical FWHM radius. The dashed lines show the median value, and the number of clumps in the subsample is shown in the upper left.
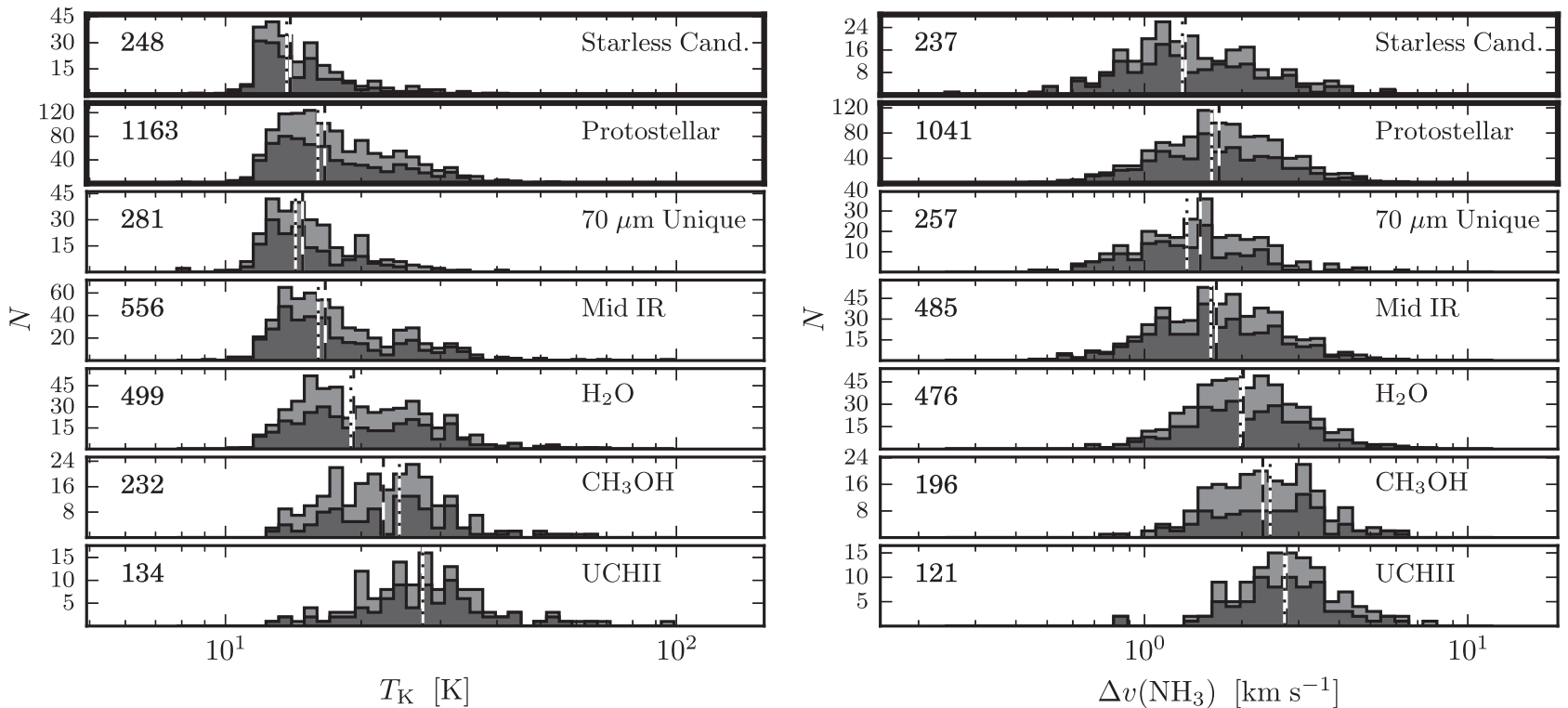

Figure 12. Observed distributions of $\mathrm{NH}_{3}$-derived kinetic temperature (left) and line width (right) by star formation indicator. The dashed lines show the median value, and the number of clumps in the subsample is shown in the upper left.

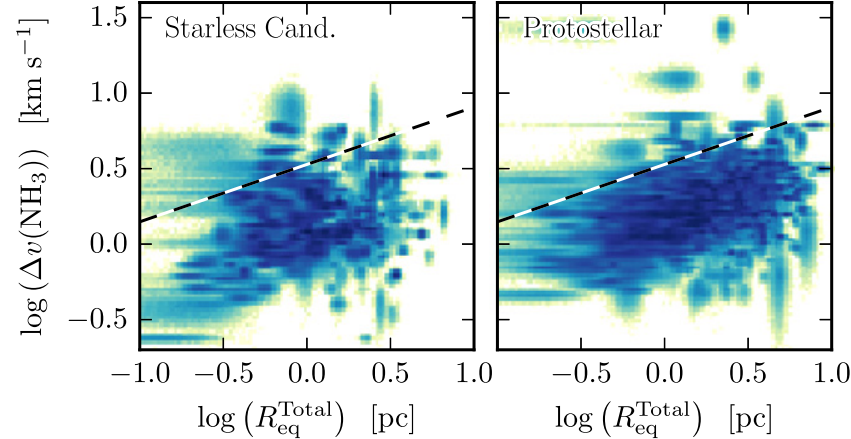

Figure 13. Line width-size relationship plotted for starless clump candidates (left) and protostellar clumps (right) drawn from MC simulations of each quantity. The protostellar clumps have a higher Spearman rank correlation coefficient $\rho_{\mathrm{sp}}=0.50$ than the starless clump candidates $\rho_{\mathrm{sp}}=0.24$. The dashed line indicates the converted relationship $\Delta v=3.37 \mathrm{~km} \mathrm{~s}^{-1}(R / \mathrm{pc})^{0.38}$ observed in Larson (1981) for molecular clouds traced by CO 1-0.

\subsubsection{Clump Mass}

We calculate total clump masses with

$$
\begin{aligned}
M_{\mathrm{H}_{2}} & =\frac{S_{1.1} d_{\mathrm{ML}}^{2}}{B_{1.1}\left(T_{\text {dust }}\right) \kappa_{\text {dust, } 1.1 \zeta}} \\
& \approx 13.1\left(\frac{e^{13 \mathrm{~K} / T_{\text {dust }}}-1}{e^{13 \mathrm{~K} / 20 \mathrm{~K}}-1}\right)\left(\frac{S_{1.1}}{1 \mathrm{Jy}}\right)\left(\frac{d_{\odot}}{1 \mathrm{kpc}}\right)^{2} M_{\odot} .
\end{aligned}
$$

Figure 15 shows the distributions of $M_{\mathrm{H}_{2}}^{\text {Total }}$ when sorted by indicator from $10^{4} \mathrm{MC}$ simulations that account for R08 AGB catalog contamination and uncertainties in $S_{1.1}, T_{\mathrm{K}}$ (or randomly drawn from the appropriate $T_{\mathrm{K}}$ distribution if no $T_{\mathrm{K}}$ observations exist), and the DPDFs. The masses show a systematic increase from $\mu_{1 / 2}\left(M_{\mathrm{H}_{2}}\right)=226 \pm 11 M_{\odot}$ for SCCs to $\mu_{1 / 2}\left(M_{\mathrm{H}_{2}}\right)=600 \pm 20 M_{\odot}$ for protostellar clumps. Among 

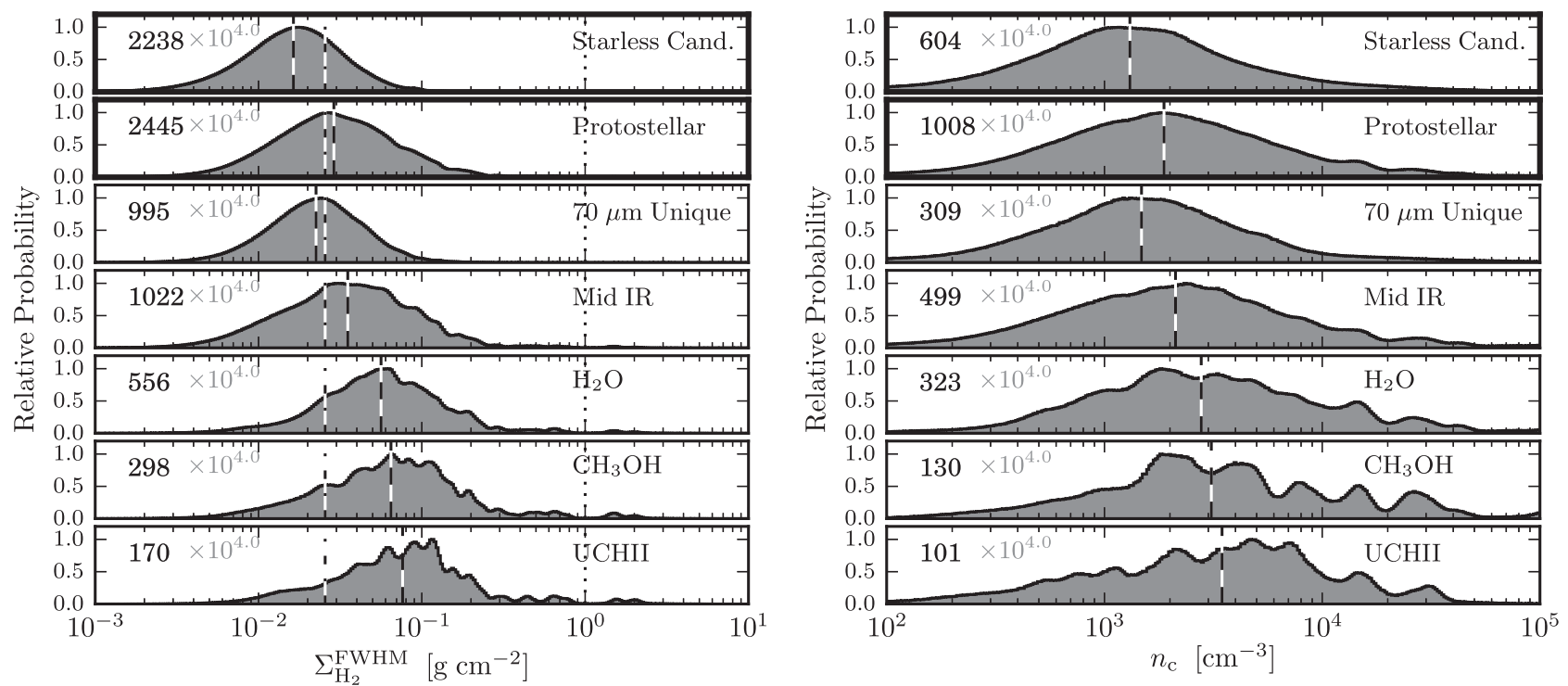

Figure 14. Distributions of FWHM mass surface (left) density and central density (right) from MC simulations by star formation indicator. The dashed lines show the median value, and the number of clumps in the subsample is shown in the upper left. The dotted line shows the predicted $1 \mathrm{~g} \mathrm{~cm}^{-3}$ threshold for the formation of a massive star from Krumholz \& McKee (2008). The dot-dashed line shows the $0.025 \mathrm{~g} \mathrm{~cm}^{-3}$ threshold for star formation observed in local clouds (Heiderman et al. 2010; Lada et al. 2010).
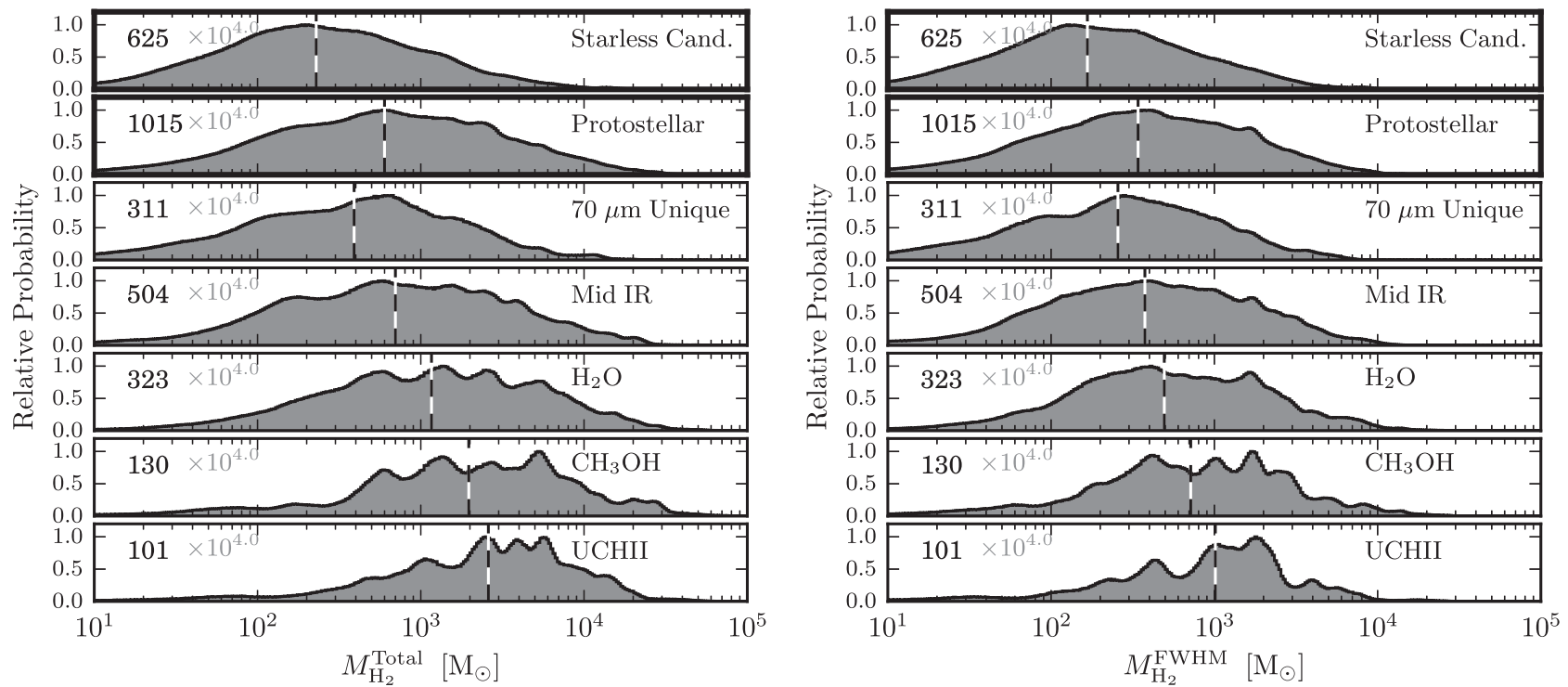

Figure 15. Distributions drawn from MC simulations of total mass (left) and FWHM mass (right) sorted by star formation indicator. The number of clumps in a sample is shown in the upper left, and a vertical dashed line shows the median value.

the clumps associated with star formation indicators, the masses increase from $\mu_{1 / 2}\left(M_{\mathrm{H}_{2}}\right)=390 \pm 30 M_{\odot}$ for the $70 \mu$ m unique category to $\mu_{1 / 2}\left(M_{\mathrm{H}_{2}}\right)=2600 \pm 200 M_{\odot}$ for the UCH II category. The difference in median masses between the SCC and protostellar categories is of $\Delta \mu_{1 / 2}\left(M_{\mathrm{H}_{2}}\right)=$ $370 \pm 20 M_{\odot}$ and the SCC and $70 \mu \mathrm{m}$ unique category is of $\Delta \mu_{1 / 2}\left(M_{\mathrm{H}_{2}}\right)=170 \pm 30 M_{\odot}$. The median mass difference is driven by more SCCs than protostellar clumps below $470 M_{\odot}$ and more protostellar clumps than SCCs above that mass. We statistically estimate the highest-mass SCC by integrating the upper tail of the $M_{\mathrm{H}_{2}}^{\text {Total }}$ PDF to where it equals one part in the sample size, 1:625, or the 99.84th percentile. This sets the maximum observed mass of SCCs at $<1.4 \times 10^{4} M_{\odot}$. The observed increase in mass toward clumps associated with more extreme star formation indicators could be due to several physical and systematic explanations. In the remainder of this section, we explore possible systematic effects to generate the observed mass difference.

For physical properties that depend on the dust temperature of the clumps, we assume an isothermal temperature with $T_{\text {dust }}=T_{\mathrm{K}}$ in the clumps. Nominally, these two temperatures are only expected to be well coupled by collisions at densities greater than $\sim 10^{5} \mathrm{~cm}^{-3}$ (Goldsmith 2001; Young et al. 2004). Battersby et al. (2014) find no correlation between $\mathrm{NH}_{3}$-derived $T_{\mathrm{K}}$ and Herschel-derived $T_{\text {dust }}$ for a quiescent clump; however, agreement within $20 \%$ is observed when the Herschel data are not background subtracted. Battersby et al. (2014) suggest 
that the gas and dust are weakly coupled at densities $10^{4}$ $10^{5} \mathrm{~cm}^{-3}$. Similarly, the $T_{\text {dust }}$ derived from far-infrared SEDs in Traficante et al. (2015a) shows a $20 \%$ agreement to $T_{\mathrm{K}}$ for both starless candidate and protostellar clumps. A $20 \%$ decrease in $T_{\text {dust }}$ (from $T_{\mathrm{K}} \sim 14$ ) will increase our mass and column density measurements for the starless candidate group by $30 \%$. This effect alone is not enough to explain the observed mass differences. There is no reason to decrease only the dust temperatures of SCCs. Any simultaneous decrease in $T_{\text {dust }}$ for protostellar clumps would mitigate the decrease of the median mass differences.

In reality, there are dust temperature gradients within the BGPS beam owing to heating from the ISRF and to embedded protostars in protostar-containing clumps. The general effect of line-of-sight dust temperature gradients on graybody SED fitting of dust continuum emission is to overestimate the dust temperature and underestimate the mass more severely for starless clumps than protostellar clumps (Malinen et al. 2011). The corresponding effect on the line-of-sight average $\mathrm{NH}_{3} T_{\mathrm{K}}$ cannot explain the observed mass difference unless the temperature gradients cause an underestimate of the average line-of-sight $T_{\mathrm{K}}$ for the SCCs from the observed median value of $14.0 \mathrm{~K}$ down to $10.0 \mathrm{~K}$, a value that is lower than all $\mathrm{NH}_{3}-$ based $T_{\mathrm{K}}$ measurements in this paper and $99 \%$ of SED-based $T_{\text {dust }}$ measurements in Traficante et al. (2015a). Analysis of the dust temperature profiles from grids of radiative transfer models of low-mass starless cores (Shirley et al. 2005; Launhardt et al. 2013) finds that the mass-weighted average $T_{\text {dust }}$ never drops below $10 \mathrm{~K}$ for a grid of Bonner-Ebert spheres (Ebert 1955; Bonnor 1956) with central densities spanning $10^{4}$ to $3 \times 10^{6} \mathrm{~cm}^{-3}$ and the strength of the ISRF equal to half the standard value (Habing 1968). These lowmass starless core models are not the exact analogs of massive starless clumps, which are possibly fragmented and clumpy, have lower observed average density (Figure 14), and are likely subject to stronger heating from the ISRF than half the Habing value, effects that will increase the average $T_{\text {dust }}$ compared to low-mass starless cores. These results indicate that it is unlikely that dust temperature gradients result in an average $T_{\text {dust }}$ as low as $10.0 \mathrm{~K}$. Radiative transfer modeling of the entire BGPS clump population is beyond the scope of the current paper and requires higher spatial resolution continuum and $\mathrm{NH}_{3}$ images. In the absence of reliable dust continuum temperatures for the full sample, a single-component $T_{\text {dust }}=T_{\mathrm{K}}$ assumption is the next best alternative.

The other assumption made in calculation of mass surface density and mass is that the dust opacities are well described by OH5 dust. They are a popular set of opacities because grains are expected to coagulate and accrete ice mantle in dense environments and there are observational constraints that indicate that $\mathrm{OH} 5$ opacities are a reasonable match, albeit toward nearby, low-mass cores (see Shirley et al. 2011). The OH5 model assumes that the grains have coagulated for a period of $10^{6} \mathrm{yr}$ at a density of $10^{5} \mathrm{~cm}^{-3}$. Both this time period and the density used in the coagulation model are likely larger than the typical values observed toward BGPS clumps (see Dunham et al. 2011a; Schlingman et al. 2011). In order to explain the observed $\Delta M_{\mathrm{H}_{2}}^{\text {Total }}$, the opacity ratio toward protostellar clumps versus starless clumps would have to be 1.7-2.6 (for $70 \mu \mathrm{m}$ unique clumps or the full protostellar clump sample). While it is certainly likely that there are opacity variations among clumps that deviate from the assumed $\mathrm{OH} 5$ opacities, the variation would have to be systematic between starless candidate and protostellar clumps. There is currently no evidence for or against such a systematic variation in opacities.

The observed mass difference cannot be due to selection effects on the flux density $S_{1.1}$ of the distance sample alone (i.e., the subsample of clumps that have DPDFs compared to those without) because SCCs and protostellar clumps have similar ratios in median $S_{1.1}$ (see Section 5, Figure 8(d)). At most this effect could account for $\sim 10 \%$, lowering the mass difference to $\Delta \mu_{1 / 2}\left(M_{\mathrm{H}_{2}}\right) \approx 330 M_{\odot}$. In addition, as a fluxlimited survey, the BGPS is effected by Malmquist bias and mass incompleteness. Ellsworth-Bowers et al. (2015) derive the mass completeness function for clumps in the distance sample by computing MC simulations drawn from the DPDFs, where the sample is $50 \%$ complete above $M_{\mathrm{H}_{2}}^{\text {Total }} \approx 70 M_{\odot}$ and $90 \%$ above $M_{\mathrm{H}_{2}}^{\text {Total }} \approx 400 M_{\odot}$. The median SCC mass, $M_{\mathrm{H}_{2}}^{\text {Total }}=230 M_{\odot}$, is at the $80 \%$ completeness level, and the $90 \%$ completeness level is achieved at the 64th mass percentile. The incompleteness in the SCC category should not, qualitatively, affect the observed median mass difference. Because $R_{\text {proto }}$ is smaller at low flux densities and masses, the population of undetected clumps with $M<400$ would disproportionately add to the SCC category and simply enhance the median mass difference further.

One additional possibility is that the spatial filtering in BGPS has systematically affected the flux densities of sources. Ginsburg et al. (2013) estimate the effects of spatial filtering by approximating the source brightness distribution as a power law with varying index and find that the flux density for sources with angular radii less than $40^{\prime \prime}$ is essentially recovered (see Ginsburg et al. 2013, Figure 8). Depending on the exact value of the power-law index, the spatial filtering recovers $>75 \%-80 \%$ of the flux density for sources with angular radii $<60^{\prime \prime}$. This accounts for $77 \%(3606 / 4683)$ of the BGPS sources. It is evident from Figure 10(a) that the sources more strongly affected by spatially filtering are protostellar stages with more luminous evolutionary indicators that tend to have larger angular radii. Thus, accounting for spatial filtering should systematically increase the median mass difference between SCCs and protostellar clumps. A detailed analysis of the effects of spatial filtering on a source-by-source basis is beyond the scope of the present work.

In summary, the observed mass difference cannot be explained by biases between the distance sample and the full sample, incompleteness, spatial filtering, or our assumptions in the mass calculation unless there is a factor of 1.7-2.6 systematic variation in dust opacities between SCCs and protostellar clumps. Interestingly, the BGPS sample is the second statistically significant sample to see this systematic offset in mass. The Herschel survey of Traficante et al. (2015a) toward IRDCs also finds a modest offset of $\sim 80 M_{\odot}$ between the average mass of starless clumps and protostellar clumps. While the mass difference is smaller by a factor of two in their study than in ours, the mass difference in their survey is significant. Alternatively, Hoq et al. (2013), using data from the first-year data release of the MALT90 molecular line mapping survey of several hundred ATLASGAL clumps in the fourth and first quadrants, do not observe an increasing trend in total mass from an " $8 / 24 \mu \mathrm{m}$ quiescent" phase to a "PDR" phase. It should be noted that these two surveys are drawn from subsets of clumps in the Galactic plane; therefore, interpretation of these results depends on how the properties of the subsets 
compare to the more complete clump populations observed in (sub)millimeter continuum Galactic plane surveys. In Section 6.3 we explore possible physical explanations for the variation in the median mass between the SCCs and protostellar candidates under the assumption that the observed mass difference is real.

\subsubsection{Virial Parameter}

The virial parameter, $\alpha=M_{\text {vir }} / M$, expresses the relative importance of gravitational and kinetic energy and can be used to assess whether clumps are gravitationally bound and/or stable to collapse. We calculate the virial parameter for a homoeoidal ellipsoid (ellipsoid of revolution) via

$$
\begin{aligned}
\alpha & =\frac{5}{8 \ln 2} \frac{1}{a_{1} a_{2}} \frac{\Delta v^{2} R}{G M} \\
& \approx 1 \frac{1}{a_{1}}\left(\frac{\Delta v}{1 \mathrm{~km} \mathrm{~s}^{-1}}\right)^{2}\left(\frac{R}{1 \mathrm{pc}}\right)\left(\frac{M}{209 M_{\odot}}\right)^{-1}
\end{aligned}
$$

where $a_{1}=(1-p / 3) /(1-2 p / 5)$ is the correction factor for a power-law density distribution with index $p$ and $a_{2} \sim 1$ for sources with aspect ratios less than 2 (Bertoldi \& McKee 1992). For a clump with negligible magnetic fields, $\alpha=1$ is gravitational virial equilibrium and $\alpha \approx 2$ is marginally gravitationally bound (see Kauffmann et al. 2013). The BGPS does not have sufficient resolution to constrain $p$, so lacking appropriate data, we draw $p=1.8 \pm 0.4$ Gaussian deviates in the MC simulations, as constrained from dust continuum modeling of high-mass star-forming regions (Mueller et al. 2002). Here we use the total mass and radius, thus setting the outer boundary to calculate $\alpha$ at the extent of the $1.1 \mathrm{~mm}$ emission. Figure 16 shows the virial parameter calculated from MC simulations for starless candidates and protostellar clumps with median values $\alpha=0.73 \pm 0.06$ and $\alpha=0.68 \pm 0.03$, respectively, suggesting that most clumps are in approximate virial equilibrium with more than half of the clumps in the distance sample with $\mathrm{NH}_{3}$ observations showing (sub)virialized motions $(\alpha \lesssim 1)$. Furthermore, $76 \%$ of the starless candidates and $86 \%$ of the protostellar clumps have $\alpha<2$, implying that most BGPS clumps are gravitationally bound. Although $24 \%$ of the starless candidates are unbound by the criteria $\alpha>2$, it is probable that these clumps host gravitationally bound higher-density substructures. These results suggest that the majority of BGPS clumps in the distance sample are self-gravitating or collapsing.

Observations of GMCs indicate virial parameters that are typically $\gtrsim 2$ (Larson 1981; Scoville et al. 1987; Solomon et al. 1987). For instance, analysis of GMCs in the GRS finds a mean value of $\alpha=1.9$ (Heyer et al. 2009). For smaller clump scales there exist a range of virial parameter measurements that report samples with $\alpha \gtrsim 1$ or $\alpha \lesssim 1$ (see Kauffmann et al. 2013 for a summary of literature values). We find our measurements of $\alpha \lesssim 1$ consistent with other Galactic plane surveys toward clumps from both BGPS and ATLASGAL with $\mathrm{NH}_{3-}$ determined line widths. The most direct comparisons are previous studies using GBT observations of $\mathrm{NH}_{3}$ that have been carried out toward the BGPS sources (Dunham et al. 2010, 2011b). Dunham et al. (2011b) measured the virial parameter toward 456 BGPS clumps with a median $\alpha=0.74$.

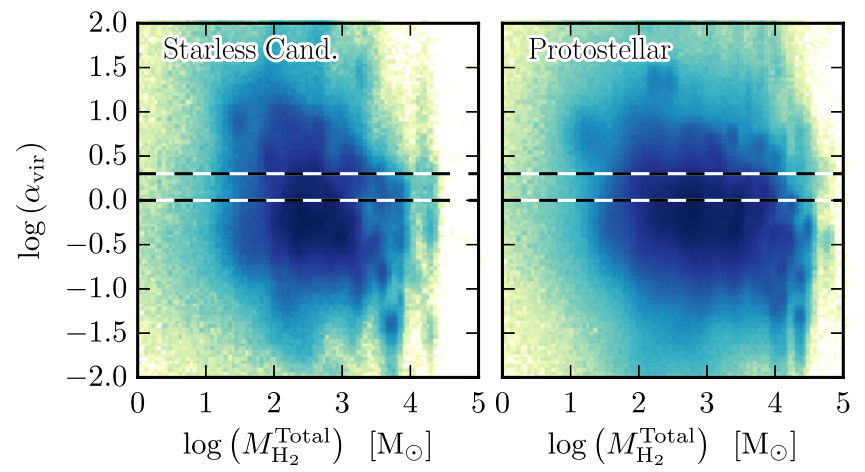

Figure 16. Comparison of virial parameter to clump total mass for SCCs (left) and protostellar clumps (right) computed using values drawn from the MC simulations. Virial parameters are derived from the model-fit $\mathrm{NH}_{3}$ velocity dispersion. The dotted lines show $\alpha=1$ and $\alpha=2$. Note the logarithmic scaling.

Wienen et al. (2012) also measure the virial parameter toward ATLASGAL clumps using $\mathrm{NH}_{3}$ and find a mean $\alpha \lesssim 1$ for clumps.

One important aspect that is often ignored in the standard virial parameter analysis is the importance of magnetic fields in virial balance. Magnetic fields in dense gas are notoriously difficult to measure, and there have been no systematic observations toward BGPS clumps. We can estimate the $B$ field strength required for virial balance from the expressions derived in Kauffmann et al. (2013) (their Equations (6) and (16)),

$$
B \gtrsim 15 \mu \mathrm{G}\left(\frac{2}{\alpha}-1\right)\left(\frac{\Delta v}{1 \mathrm{~km} \mathrm{~s}^{-1}}\right)^{2}\left(\frac{1 \mathrm{pc}}{R}\right) .
$$

Evaluating the above expression for the median values of the SCCs indicates that $B$-fields of only $50 \mu \mathrm{G}$ are needed to support typical SCCs against collapse. Magnetic fields of this magnitude and larger are indeed observed toward IRDCs. For instance, Pillai et al. (2015) find a total $B$-field strength of $\sim 250 \mu \mathrm{G}$ toward the IRDC G11.1 from analysis of submillimeter continuum polarization. The required $B$-field strength scales as $B \sim 1 / \alpha$, implying that very subvirial clumps with $\alpha \lesssim 0.1$ required $B \gtrsim 600 \mu \mathrm{G}$. In these more extreme cases, $B$ field support may prove difficult, but for the typical (medianproperty) SCCs, the required $B$-field strength for support is within a reasonable range of observed values (for a summary of $B$-field measurements, see Crutcher 2012).

\section{DISCUSSION}

For many of the observed properties and calculated physical properties analyzed in Section 5, there is a systematic trend in the property with the ordering of the star formation indicators from $70 \mu \mathrm{m}$ unique flags, to mid-IR star formation flags, to $\mathrm{H}_{2} \mathrm{O}$ maser flags, to $\mathrm{CH}_{3} \mathrm{OH}$ flags, to $\mathrm{UCH}$ in flags. Protostellar clumps that contain the most extreme indicators of luminous protostars $\left(\mathrm{CH}_{3} \mathrm{OH}\right.$ maser and $\mathrm{UCH}$ II containing clumps $)$ tend to have the highest flux densities, are the most centrally condensed, have the highest temperatures, and are more turbulent and more massive on median than other clumps. A partial evolutionary analysis of the ATLASGAL clumps associated with RMS sources, $\mathrm{CH}_{3} \mathrm{OH}$ maser, and $\mathrm{UCH}$ II regions has been performed (Urquhart et al. 2011, 2013a, 2013b, 2014). The BGPS results are consistent with the 


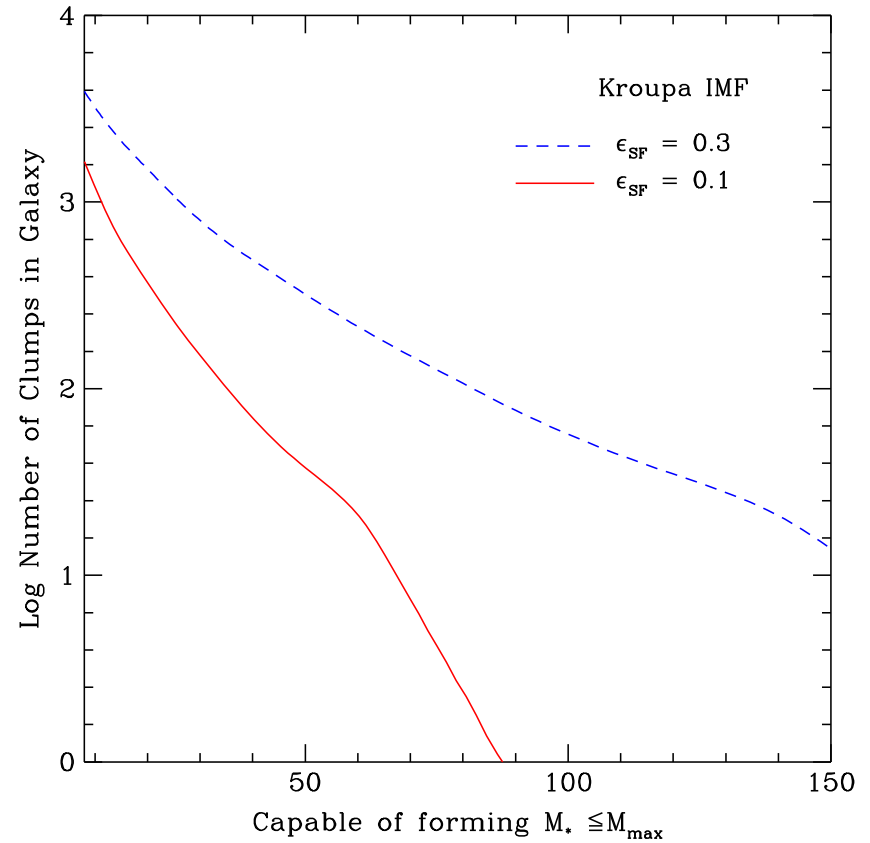

Figure 17. Number of clumps in the Milky Way capable of forming a star with a mass $\leqslant M_{\max }$ assuming a Kroupa IMF. Two star formation efficiencies are plotted for the clumps ( 0.1 in red solid line and 0.3 in blue dashed line).

observed trends $\left(M, T_{\mathrm{K}}\right)$ for the properties of ATLASGAL clumps that contain methanol masers and $\mathrm{H}_{\text {II }}$ regions (see Table 4 of Urquhart et al. 2014). In contrast, the observable and physical properties indicate that SCCs have lower flux densities, are less centrally concentrated, have smaller sizes, are colder and less turbulent, and are less massive on median when compared to clumps with indications of protostellar activity. In this section, we explore the potential for forming massive stars, the timescales for evolution of this newly discovered population of starless clumps, and possible explanations for the observed median mass difference between starless and protostellar clumps.

\subsection{Massive Star-forming Potential of Clumps}

With a robust sample of SCCs, it is important to assess their potential to form high-mass stars. We can estimate the mass of the most massive star formed by an SCC, $M_{\max }$, using the stellar IMF from Kroupa (2001). ${ }^{13}$ The total stellar cluster mass is equal to the mass of the progenitor clump times a star formation efficiency factor $\epsilon_{\mathrm{SF}}$. We can derive a relationship between $M_{\max }$ and the mass of the clump from

$$
\begin{gathered}
\epsilon_{\mathrm{SF}} M_{\text {clump }}=\frac{\int_{0.08}^{150} N(M) M d M}{\int_{M_{\max }}^{150} N(M) d M} \\
M_{\max } \approx 20 M_{\odot}\left(\frac{\epsilon_{\mathrm{SF}}}{0.3} \frac{M_{\text {clump }}}{1064 M_{\odot}}\right)^{1 / 1.3},
\end{gathered}
$$

where the IMF is normalized such that at least one star with $M \geqslant M_{\max }$ is formed. The values of $\epsilon_{\mathrm{SF}}$ range from 0.05 to 0.5 with a typical value of 0.3 (see Lada \& Lada 2003; Shirley

\footnotetext{
${ }^{13} N(M) \propto M^{-1.3} \quad$ for $\quad 0.08 M_{\odot} \leqslant M \leqslant 0.5 M_{\odot}, \quad N(M) \propto M^{-2.3} \quad$ for $M>0.5 M_{\odot}$. We assume a maximum stellar mass of $150 M_{\odot}$ in IMF calculations.
}

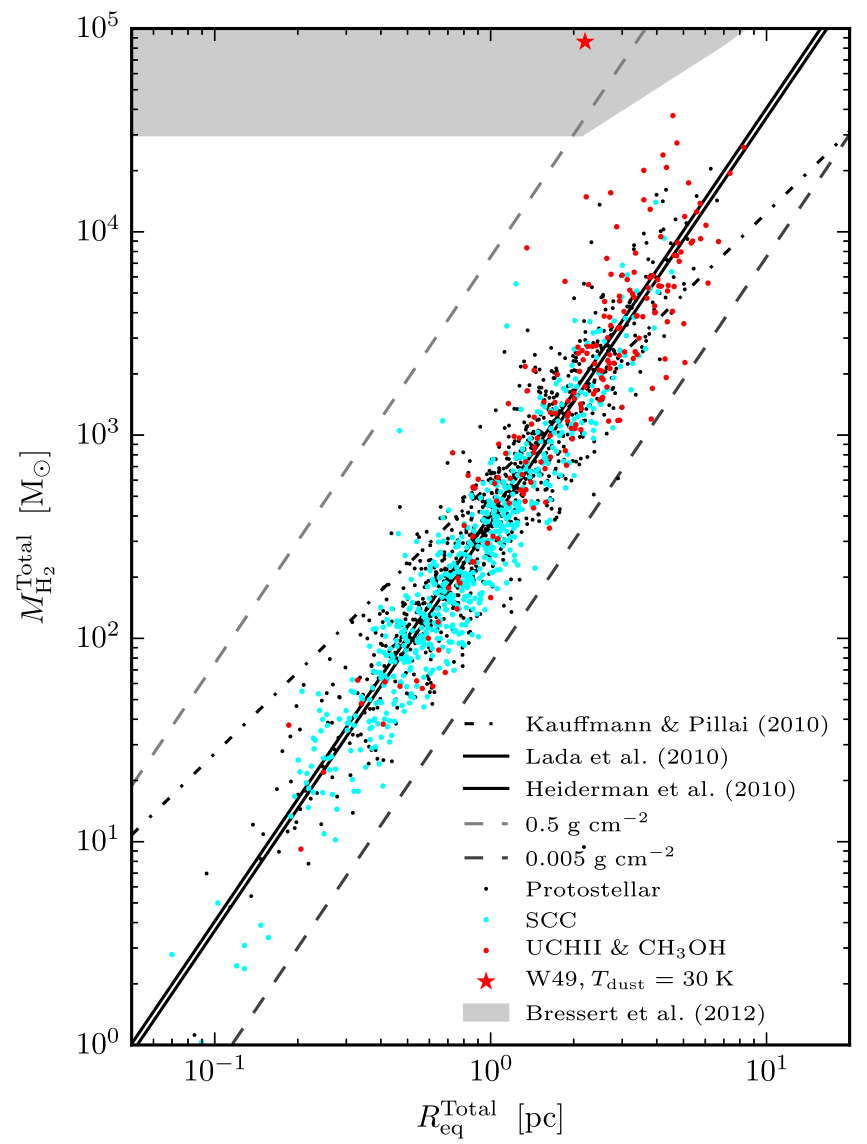

Figure 18. Equivalent radius compared to total mass. Median values for each clump are computed from MC simulations. Clumps are shown associated with $\mathrm{UCH}$ II or $\mathrm{CH}_{3} \mathrm{OH}$ masers (red), other protostellar (black), and SCCs (cyan). The dot-dashed line shows the threshold for high-mass star formation $M(R) \geqslant 580 M_{\odot}\left(R \mathrm{pc}^{-1}\right)^{1.33}$ from Kauffmann \& Pillai (2010) when scaled to OH5 dust opacities. Solid lines show thresholds for "effecient star formation" of $116 M_{\odot} \mathrm{pc}^{-2}$ (Lada et al. 2010) and $129 M_{\odot} \mathrm{pc}^{-2}$ (Heiderman et al. 2010). The upper and lower dashed gray lines show 0.5 and $0.005 \mathrm{~g} \mathrm{~cm}^{-2}$, respectively. The shaded gray region in the upper left indicates the part of parameter space for YMCs as defined in Bressert et al. (2012).

et al. 2003; Krumholz et al. 2007; Bontemps et al. 2010; Kuiper et al. 2010). Using $8 M_{\odot}$ as the definition of the minimum mass of a high-mass star, the corresponding BGPS clump mass is $320 M_{\odot}$. In the distance sample $42 \%$ of SCCs (264 clumps) have masses above $320 M_{\odot}$ in the MC simulations. This corresponds to 603 SCCs in the BGPS volume if we assume that the SCCs without well-constrained DPDFs have a similar average DPDF and account for the different flux density distributions. If we use the Wolfire et al. (2003) model of the $\mathrm{H}_{2}$ distribution in the Galaxy as an axisymmetric proxy for the massive clump spatial distribution, ${ }^{14}$ then there are $\sim 3900$ SCCs capable of forming an $8 M_{\odot}$ star in the entire Milky Way.

\footnotetext{
${ }^{14}$ The correction factor is calculated numerically by integrating the $\mathrm{H}_{2}$ density distribution over the volume of the wedge observed by the BGPS divided by the integral of the $\mathrm{H}_{2}$ density distribution over the total volume of the Galaxy. The Wolfire et al. (2003) density distribution is given by $\rho\left(R_{\mathrm{g}}, z_{\mathrm{g}}\right) \propto \exp \left(-4 \ln 2\left(R_{\mathrm{g}}-0.571 R_{\odot}\right)^{2} /\left(0.52 R_{\odot}\right)^{2}\right) \exp \left(-\left|z_{\mathrm{g}}\right| / 0.059\right)$ for $R<0.82 R_{\odot}$ and $\rho\left(R_{\mathrm{g}}, z_{\mathrm{g}}\right) \propto \exp \left(-R_{\mathrm{g}} /\left(0.34 R_{\odot}\right)\right) \exp \left(-\left|z_{\mathrm{g}}\right| / 0.059\right)$ for $R \geqslant 0.82 R_{\odot}$ where $R_{\mathrm{g}}$ is the galactocentric radius in kpc, $z_{\mathrm{g}}$ is the height above the Galactic midplane in $\mathrm{kpc}$, and $R_{\odot}=8.5 \mathrm{kpc}$ is the distance to the Galactic center. Note that we account for the vertical offset of $25 \mathrm{pc}$ of the Sun above the Galactic plane in the calculation of the BGPS wedge integrals (see Appendix C of Ellsworth-Bowers et al. 2013).
} 


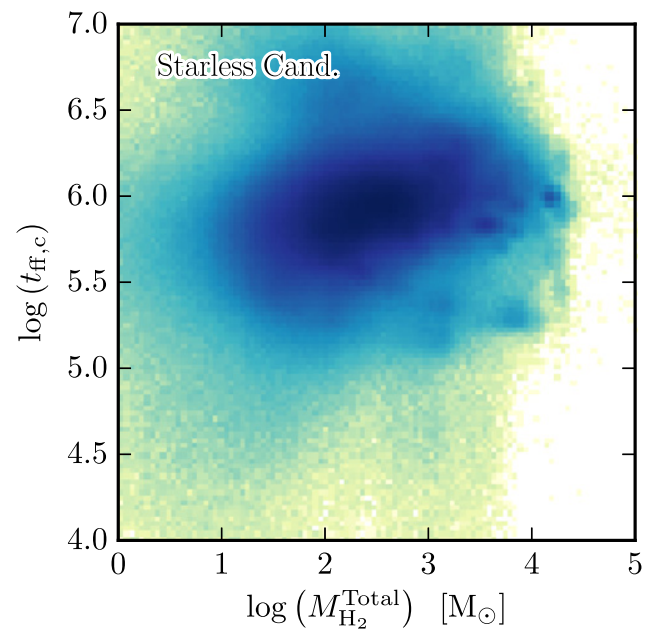

Figure 19. Comparison of clump freefall time to clump total mass for starless clump candidates computed using values drawn from the MC simulations. Freefall time values are calculated from central density $n_{\mathrm{c}}$.

Figure 17 plots the number of clumps in the Milky Way capable of forming stars with masses $\leqslant M_{\max }$. Note that the number of clumps is very sensitive to assumed star formation efficiency, especially for very massive stars.

An alternative way to assess the massive star-forming potential of BGPS clumps is to compare $M_{\mathrm{H}_{2}}$ and $R_{\mathrm{eq}}$ (Figure 18). Values are represented as the median value drawn from the MC simulations per clump. A robust increasing correlation is observed with $\rho_{\mathrm{sp}}=0.942$. To show the differences in clumps by star formation activity, we plot SCCs in yellow and clumps associated with $\mathrm{UCH}$ II or $\mathrm{CH}_{3} \mathrm{OH}$ masers as unambiguous detections of high-mass star formation activity in red. The distributions of points follow the general trends discussed in Section 5: SCCs are generally lower mass, smaller radius, and lower mass surface density. Several thresholds for star formation determined from local clouds are overplotted. Fitting star formation rates to local molecular clouds, Lada et al. (2010) and Heiderman et al. (2010) find relationships for "effecient" star formation of $\approx 116 M_{\odot} \mathrm{pc}^{-2}$ and $129 \pm M_{\odot} \mathrm{pc}^{-2}$, respectively, where above these thresholds the star formation rate is linearly proportional to mass surface density. The thresholds from Lada et al. (2010) and Heiderman et al. (2010) approximately bisect the samples, with $50.6 \%$ and $42.5 \%$ of the full samples, $46.6 \%$ and $39.9 \%$ of the $\mathrm{UCH}$ II and $\mathrm{CH}_{3} \mathrm{OH}$ maser sample, and $41.4 \%$ and $33.6 \%$ of the SCC sample exceeding the respective limits. Kauffmann \& Pillai (2010) derive the more restrictive criteria of $M \leqslant 580 M_{\odot}\left(R_{\mathrm{eq}} / \mathrm{pc}\right)^{1.33}$ from observations of nearby molecular clouds. Note that Kauffmann \& Pillai (2010) scale their OH5 dust opacities down by a factor of 1.5, so for consistency to values in this work we use the leading factor of $580 \mathrm{M}_{\odot}$ rather than the original $870 M_{\odot}$. In the BGPS distance sample, $24.8 \%$ of the full sample, $54.9 \%$ of the $\mathrm{UCH}$ II and $\mathrm{CH}_{3} \mathrm{OH}$ maser sample, and $10.8 \%$ of the SCC sample exceed this criterion. Because nearly half of BGPS clumps with an unambiguous detection of a high-mass YSO do not meet this criterion, this suggests that the BGPS cloud structures do not strictly follow the criteria derived in Kauffmann \& Pillai (2010). While the majority of SCCs lie below this threshold, any systematic growth would increase clump total mass and radius, placing them in phase space for a higher probability of high-mass star formation (see Section 6.3.2). In a similar blind sample from the ATLASGAL survey, Wienen et al. (2015) find that $92 \%$ of ATLASGAL clumps meet this criterion and $100 \%$ above the Lada et al. (2010) and Heiderman et al. (2010) lines. Notably, only one clump in the BGPS from $10^{\circ}<\ell<65^{\circ}$ (W49, G043.167+00.011) meets the criteria for the progenitors of young massive clusters (YMCs; Longmore et al. 2014, p. 291) with clump masses $\gtrsim 10^{5} M_{\odot}$, although W49 is not an SCC because it is actively forming stars.

\subsection{Clump Timescales}

\subsubsection{Average Freefall Time}

For the SCC sample we estimate the average freefall time, $t_{\mathrm{ff}}$, calculated with

$$
\begin{aligned}
t_{\mathrm{ff}} & =\sqrt{\frac{3 \pi}{32 G \mu m_{\mathrm{p}} n_{c}}} \\
& \approx 0.98\left(\frac{n_{\mathrm{c}}}{10^{3} \mathrm{~cm}^{-3}}\right)^{-1 / 2} \mathrm{Myr},
\end{aligned}
$$

where $n_{\mathrm{c}}$ is the clump central density. We estimate the clump central density $n_{\mathrm{c}}$ from the peak mass surface density and FWHM radius, assuming a spherically symmetric Gaussian density distribution (cf. Appendix C of Pattle et al. 2015). Integrating over the FWHM cylindrical aperture yields the expression

$$
n_{\mathrm{c}}=\sqrt{\frac{\ln 2}{\pi}} \frac{\Sigma_{\mathrm{H}_{2}}^{\text {peak }}}{R^{\text {FWHM }}}\left[\operatorname{erf}\left(\sqrt{\ln 2} \frac{\theta^{\text {Total }}}{\theta^{\text {FWHM }}}\right)\right]^{-1}
$$

where $\theta$ is the deconvolved angular radius for the total or FWHM definition (see Section 5.2). While a density distribution described by a singly peaked Gaussian is a crude assumption, it is more representative of higher-density substructures within starless clumps than just the average density with the FWHM. Figure 19 shows the average freefall time plotted versus the mass of the clumps of the MC simulation. There is no significant correlation between the average freefall time and the mass of the clumps. The median is $t_{\mathrm{ff}, \mathrm{c}}=0.84 \pm 0.02 \mathrm{Myr}$ for the SCCs.

Since the BGPS clump FWHM sizes are typically a factor of two larger when compared to the Jeans length of $0.36 \mathrm{pc}\left(T_{\mathrm{K}} / 15 \mathrm{~K}\right)^{1 / 2}\left(10^{3} \mathrm{~cm}^{-3} / n_{\mathrm{c}}\right)^{1 / 2}$, these starless clumps should fragment into smaller structures. Higher-resolution observations of a subset of BGPS clumps show this fragmentation into objects with higher densities (Merello et al. 2015). As a result, the median $t_{\mathrm{ff}}$ is likely an upper limit to the freefall time for a typical SCC to form a single detectable star.

\subsubsection{Clump Lifetimes from Number Statistics}

If the Galactic population of clumps is in steady state where the clump formation rate is equal to the destruction rate, then the number fraction of clumps in a given state is proportional to the lifetime of that state. We find nearly equal numbers of SCCs $(47.5 \%)$ compared to protostellar clumps. This result implies that the typical lifetime of an SCC is nearly equal to the typical lifetime of a protostellar clump. The starless clump lifetime is defined as the time it takes for a clump detectable by the BGPS to form a single star that is detectable in current 
infrared surveys of the Galactic plane. The protostellar clump lifetime is determined by the time it takes a cluster of stars to form (not a single star) and dissipate the gas and dust to the point where the clump is no longer detectable in the BGPS. The latter timescale is difficult to determine, but we can use the cluster formation timescale as a rough estimate. This cluster formation timescale has a large range and is typically longer than 1 Myr and up to a few megayears (Rebull et al. 2007; Chomiuk \& Povich 2011; Morales et al. 2013; see review by Longmore et al. 2014, p. 291). The total population statistics imply a long average timescale for SCCs that is on the order of a few times the average freefall time of the clumps and that is consistent with the effects of turbulence and or magnetic fields lengthening the starless evolution timescale.

A single timescale cannot properly describe the starless clump evolution timescale because clumps with different masses should evolve out of the starless phase at different rates. While a median-mass SCC $\left(230 M_{\odot}\right)$ may have a lifetime over $1 \mathrm{Myr}$, it seems highly unlikely that $10^{4} M_{\odot}$ starless clumps exist in a starless phase for that long. The lack of very massive $\left(M>1.4 \times 10^{4} M_{\odot}\right)$ SCCs argues for a shorter timescale for these objects to form a single detectable star and move to the protostellar clump category. Current singletimescale estimates (or upper limits) in the literature for massive starless clumps (Csengeri et al. 2014; Traficante et al. 2015a) are problematic because they do not account for this variation with mass and because they also attempt to compare to a single timescale for the lifetime of the embedded massive star formation phase (i.e., Davies et al. 2011; DuarteCabral et al. 2013). For the remaining discussion, we analyze how starless clump lifetimes vary with the mass of the clumps.

We can estimate how the clump lifetime for massive clumps varies with mass by using a method pioneered in Battersby (2013) by pinning the relative lifetimes of clumps to an estimated absolute lifetime of the Class II $\mathrm{CH}_{3} \mathrm{OH}$ maser $\tau_{\mathrm{CH}_{3} \mathrm{OH}}=2.5-4.5 \times 10^{4}$ year (Van der Walt 2005). This timescale is a statistical estimate based on correcting the number of the observed $\mathrm{CH}_{3} \mathrm{OH}$ masers in Pestalozzi et al. (2005) for completeness to predict the total Galactic count of high-mass stars with mass $>20 M_{\odot}$. Van der Walt (2005) uses an $\mathrm{SFR}=4 M_{\odot} \mathrm{yr}^{-1}$ based on the SFR $=3-6 M_{\odot} \mathrm{yr}^{-1}$ range in Boissier \& Prantzos (1999). Chomiuk \& Povich (2011) reanalyze several Galactic SFR measurements (see Table 1 therein) to derive $\mathrm{SFR}=1.9 \pm 0.4 M_{\odot} \mathrm{yr}^{-1}$. The Chomiuk \& Povich (2011) study is unique in that they apply the same IMF (Kroupa 2001) and SFR law to all measurements and use the Starburst99 code to self-consistently compare studies with substantially different methodologies (including free-free radio continuum observations, as well as YSO counts). Scaling the $\mathrm{CH}_{3} \mathrm{OH}$ maser lifetime inversely by their revised SFR yields $\tau_{\mathrm{CH}_{3} \mathrm{OH}}=(6.1-9.3) \times 10^{4} \mathrm{yr}$.

The clump lifetime is calculated using the relative number fraction of sources compared to the $\mathrm{CH}_{3} \mathrm{OH}$ maser sample at a given mass via

$$
\tau_{\mathrm{SCC}}=\tau_{\mathrm{CH}_{3} \mathrm{OH}}\left(\frac{N_{\mathrm{SCC}}}{N_{\mathrm{CH}_{3} \mathrm{OH}}}\right)
$$

where $\tau_{\mathrm{CH}_{3} \mathrm{OH}}$ is the completeness-corrected and the SFRcorrected Class II $\mathrm{CH}_{3} \mathrm{OH}$ maser lifetime. The ratio of $N_{\mathrm{SCC}} / N_{\mathrm{CH}_{3} \mathrm{OH}}$ is corrected for the fraction of SCCs and clumps with $\mathrm{CH}_{3} \mathrm{OH}$ that are in the distance sample compared to the

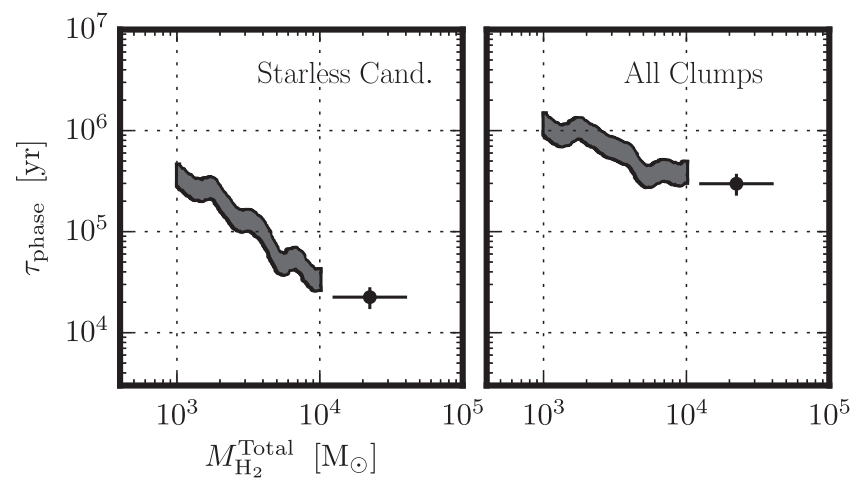

Figure 20. Clump lifetimes as a function of total mass. Relative lifetimes are computed from the number of $\mathrm{MC}$ samples in a narrow mass range and scaled using an absolute lifetime for the Class II $\mathrm{CH}_{3} \mathrm{OH}$ maser, in the range (6.1-9.3) $\times 10^{4} \mathrm{yr}$, corresponding to the upper and lower limits of the filled curves. The markers show the average values for $M>10^{4} M_{\odot}$ where $N \lesssim 30$.

total sample. The fundamental assumption of this method is that all clumps capable of forming a $20 M_{\odot}$ star $\left(M_{\text {clump }}>1000 M_{\odot}\right)$ will at some time form high-mass YSOs with detectable $\mathrm{CH}_{3} \mathrm{OH}$ masers. A population of equal-mass clumps that are not capable of producing observable $\mathrm{CH}_{3} \mathrm{OH}$ masers would decrease the observed fraction and lead us to overestimate the inferred lifetime. This method also assumes the lifetime of a single $\mathrm{CH}_{3} \mathrm{OH}$ maser $\left(\tau_{\mathrm{CH}_{3} \mathrm{OH}}\right)$ for all clumps with $\mathrm{CH}_{3} \mathrm{OH}$ maser associations. For clumps associated with multiple $\mathrm{CH}_{3} \mathrm{OH}$ maser sites (i.e., YSOs) an age spread within the clump would increase the observed duration of the clump $\mathrm{CH}_{3} \mathrm{OH}$ maser phase and lead us to underestimate the phase lifetime when the shorter, single YSO $\tau_{\mathrm{CH}_{3} \mathrm{OH}}$ is applied; however, for clumps associated with MMB sources, which have accurate interferometric positions, only 25/272 (9.2\%) are host to more than one $\mathrm{CH}_{3} \mathrm{OH}$ maser, so this does not likely introduce a strong bias. To calculate the lifetimes as a function of mass, we extend this method to the relative fractions of $\mathrm{MC}$ samples in narrow mass bins $(0.0125 \mathrm{dex}$ logarithmic bins for $10^{4} \mathrm{MC}$ mass samples per clump). In effect, this is the ratio of two mass PDFs each scaled by the sample sizes and then multiplied by $\tau_{\mathrm{CH}_{3} \mathrm{OH}}$.

Figure 20 shows the lifetime of SCCs as a function of mass. The starless candidate clump lifetime approximately follows $\tau_{\text {SCC }} \sim 0.37 \pm 0.08 \operatorname{Myr}\left(10^{3} M_{\odot} / M\right)$ for $M>10^{3} M_{\odot}$. The uncertainty corresponds to the systematic uncertainty in $\tau_{\mathrm{CH}_{3} \mathrm{OH}}$. It is not advisable to continue our timescale analysis to $<1000 M_{\odot}$ as the assumption that all clumps will form a $\mathrm{CH}_{3} \mathrm{OH}$ maser is less likely to be true; however, extrapolation of the trend to lower masses indicates that the starless clump lifetime becomes longer than the average clump freefall time at $<450 M_{\odot}$. This is approximately twice the median SCC mass. Thus, most SCCs have lifetimes longer than their average freefall times of $0.8 \mathrm{Myr}$. In turn, very massive SCCs with $M_{\mathrm{H}_{2}}>10^{4} M_{\odot}$ have very short lifetimes $\tau \lesssim 0.03$ Myr consistent with lack of such massive objects detected in BGPS (Ginsburg et al. 2012). Our lifetime estimates are 5 times longer and 3.5 times longer than the lifetime estimates of Tackenberg et al. (2012) for ATLASGAL clumps capable of forming 20 and $40 M_{\odot}$ stars, respectively. The Tackenberg et al. (2012) estimates are based on large extrapolations from small numbers 


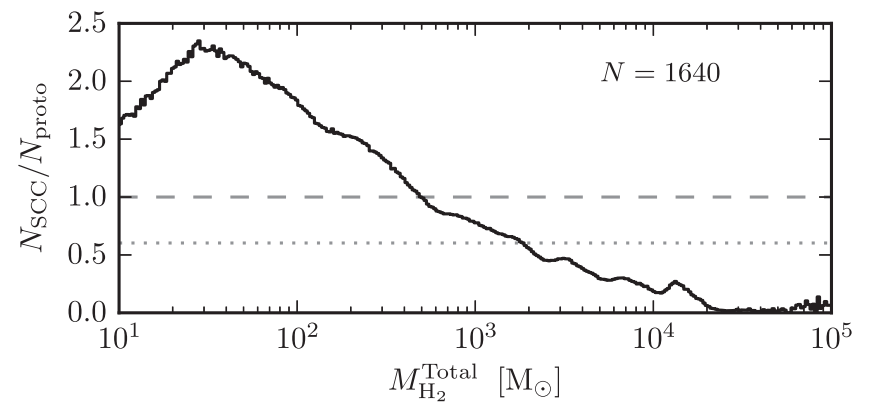

Figure 21. Ratio of starless clump candidates to protostellar clumps by total clump mass drawn from the MC simulations. The dotted line shows the total sample fraction $613 / 1027 \approx 0.597$. The plotted values taken from the distance sample are corrected by the factor $1027 / 613 \approx 1.675$ to be representative of the full survey.

of clumps (six and one, respectively) observed in their $20 \mathrm{deg}^{2}$ survey area to the entire Galaxy.

\subsection{Origin of Starless versus Protostellar Clump Mass Difference}

In Section 5.4.2 a systematic difference between the median mass of protostellar clumps and SCCs of 170-370 $M_{\odot}$ was discussed. Fundamentally, the median mass difference is due to more SCCs with masses below $M \lesssim 470 M_{\odot}$ and fewer highermass SCCs than protostellar clumps (see Figure 21). We explore three possible physical explanations for this mass difference: (i) not all SCCs will evolve into detectable protostellar clumps, (ii) clumps grow in mass by accreting surrounding material from their parent GMC, and (iii) clumps evolve at different rates from the starless to protostellar phase in exact proportion to their number statistics.

\subsubsection{Infertile Starless Clumps}

Perhaps the simplest physical explanation for the systematic mass difference is that there is a large population of clumps among the SCCs that, while starless, will not evolve to form YSOs detectable by our star formation indicators. In order for this population of objects to remain SCCs, they either would remain "infertile" and never form stars or would only be able to form stars with bolometric luminosities that are less than the $30-140 L_{\odot}$ sensitivity (corresponding to $1-2 M_{\odot}$ protostars accreting at typical rates) of current far-infrared Galactic plane surveys (Section 3). Such a population of low-density and lowmass clumps would bias the SCC distribution to lower masses. For a Kroupa IMF, a $230 M_{\odot}$ median mass SCC is capable of forming stars up to $6 M_{\odot}$, which is detectable given the sensitivity limits of current far-infrared surveys (see Figure 4); however, for SCCs at the 50\% BGPS mass completeness limit of $70 M_{\odot}$, the maximum-mass star formed is only $2.5 M_{\odot}$, which is closer to the sensitivity limit of current mid- and far-infrared surveys.

One test of this hypothesis is to check for the presence of dense gas. The $\mathrm{HCO}^{+} 3-2$ transition has an effective excitation density $n_{\text {eff }}>10^{4} \mathrm{~cm}^{-3}$ for the typical properties of SCCs (Shirley 2015). $\mathrm{HCO}^{+} 3-2$ from Shirley et al. (2013) was detected toward $44 \%$ of SCCs and $78 \%$ of protostellar clumps in the distance sample. This result indicates either that there is a population of lower-density SCCs or that their filling fraction of dense gas is less than toward protostellar clumps. Since the 3-2 transition has an upper energy level that is $25.7 \mathrm{~K}$ above ground, the effective excitation density $\left(n_{\text {eff }}\right)$ is a very sensitive function of $T_{\mathrm{K}}$ at the median temperature of $14.0 \mathrm{~K}$ measured toward SCCs. The sensitivity of the 3-2 transition to $T_{\mathrm{K}}$ means that the detection is biased against the SCCs that are colder on average than protostellar clumps. So this test is biased, meaning that since SCCs are colder on average than protostellar clumps, $\mathrm{HCO}^{+} 3-2$ requires higher densities in SCCs (by a factor of $\approx 2$ ) to be excited than toward protostellar clumps.

Another test of this hypothesis is to carefully control systematic effects from sampling clumps with different physical properties by performing a variety of astrophysical cuts on the sample and calculate the distribution of $\Delta \mu_{1 / 2}\left(M_{\mathrm{H}_{2}}\right)$ from the MC simulations. These cuts are designed to remove low-mass, low-density, or unbound objects. Figure 22 shows the cumulative distribution functions (CDFs) for $\Delta \mu_{1 / 2}\left(M_{\mathrm{H}_{2}}\right)$ subsamples selected on mass, $\mathrm{NH}_{3}$-derived gas kinetic temperature, heliocentric distance, virial parameter, mass surface density, central density, and molecular detections. The observed mass differences between categories, $\Delta \mu_{1 / 2}\left(M_{\mathrm{H}_{2}}\right)$, are robust to these criteria, with the values calculated with respect to the Hi-GAL $70 \mu \mathrm{m}$ unique category at $\Delta \mu_{1 / 2}\left(M_{\mathrm{H}_{2}}\right) \sim 100-200 M_{\odot}$ and the values calculated with respect to the protostellar category in the range $\Delta \mu_{1 / 2}\left(M_{\mathrm{H}_{2}}\right) \sim 200-500 M_{\odot}$. The robustness of the median mass difference to astrophysical cuts does not support the explanation that a population of low star formation potential SCCs solely drives the increasing trend in clump total mass.

\subsubsection{Possible Clump Mass Growth}

BGPS clumps commonly reside in a complex structural hierarchy of clouds and filaments. When sorted by protostellar indicator, the increasing trend in both $\Sigma$ and $M_{\text {tot }}$ but weak increasing trend in $R_{\text {eq }}$ is consistent with the physical process of clumps, as focusing points of local gravitational potential minima, accreting mass from the surrounding cloud in a "conveyor belt" fashion while maintaining the same approximate volume. Clump mass growth via accretion from the surrounding molecular cloud is a possible mechanism for the difference between SCCs and protostellar clump masses. An upper limit to the clump accretion rate can be set by the freefall time of the highest-density subcomponent of the clump. An estimate of $t_{\mathrm{ff}}$ from the $\mathrm{MC}$ simulation shown in Figure 19 suggests a median $t_{\mathrm{ff}}=0.84 \mathrm{Myr}$. For a median mass difference between the SCC and $70 \mu \mathrm{m}$ unique clumps or protostellar clumps of $170-370 M_{\odot}$, this yields an accretion rate of $\approx 200-440 M_{\odot} \mathrm{Myr}^{-1}$ as an upper limit if this is assumed to be the sole explanation for the mass difference. Note that all molecular clouds cannot be collapsing on freefall timescales and still be consistent with the observed Milky Way SFR (Zuckerman \& Evans 1974). Other mechanisms such as magnetic and turbulent support would increase the clump collapse time and decrease the predicted growth rate.

As a simple estimate, we calculate the total mass available to a clump embedded in a GMC with typical properties. We assume a GMC with a spherical inflow velocity of $\Delta v \sim 1 \mathrm{~km} \mathrm{~s}^{-1}$ onto a clump, feeding from a zone of $\Delta R_{\text {feed }} \sim 1.0$ pc over $t \sim 1$ Myr. For a median-sized clump with $R_{\mathrm{eq}} \sim 1 \mathrm{pc}$ and feeding zone density $n \sim 150 \mathrm{~cm}^{-3}$ (N.B. median $n=230 \mathrm{~cm}^{-3}$ in GMCs in the GRS; Roman-Duval et al. 2009), the homogeneous, spherical-shell reservoir mass would be $M_{\text {res }} \sim 250 M_{\odot}$. Higher inflow velocity or density 


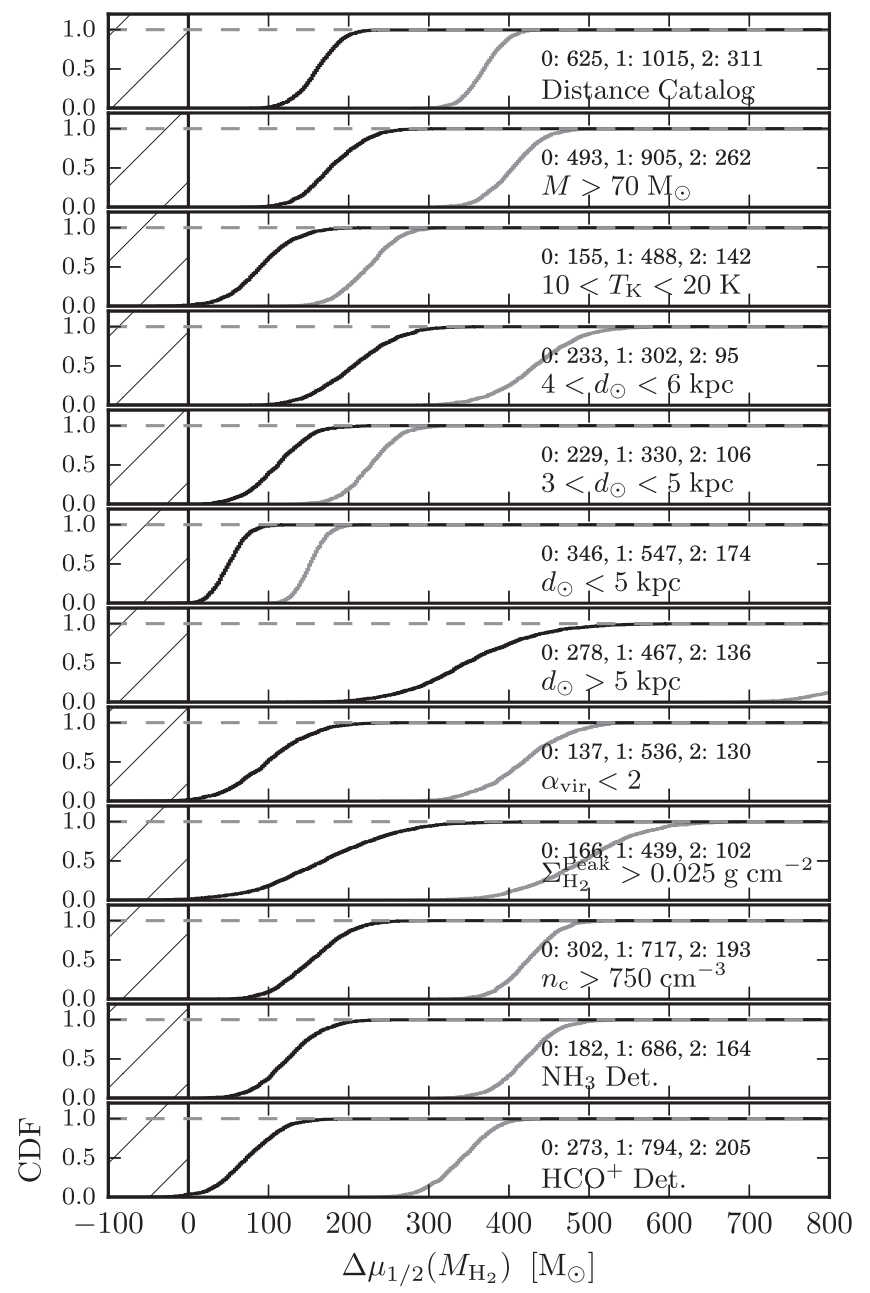

Figure 22. CDFs of the median mass difference $\Delta \mu_{1 / 2}\left(M_{\mathrm{H}_{2}}\right)$ drawn from the MC simulations based on different cuts in physical properties. The black curve shows the difference between the Hi-GAL unique and SCCs, and the gray curve shows the difference between the protostellar and SCCs. The cut is labeled in the bottom right of each panel, and above are the sample numbers for SCC (0), protostellar (1), and Hi-GAL $70 \mu \mathrm{m}$ unique (2). The values $\Delta \mu_{1 / 2}\left(M_{\mathrm{H}_{2}}\right) \sim 100 M_{\odot}$ agree across a wide range of physical cuts designed to remove low-mass, low-density, or unbound objects.

would both increase the available mass. These values show that reasonable physical conditions within a GMC yield estimates that are consistent with the observed median mass difference between the SCCs and protostellar clumps, suggesting that cloud-to-clump accretion is a plausible physical mechanism.

There are theoretical models of such large-scale clump accretion. For instance, Vázquez-Semadeni et al. (2009) invoke a model of hierarchical gravitational fragmentation in which multiscale collapse occurs. In this picture, the mass accretion rate onto the clumps is determined by the clump tidal radius and initial gravitational well hierarchy (see also Smith et al. 2009). In another approach, Murray \& Chang (2012) have explored a model of Bondi-Hoyle accretion of clumps within GMCs and predict that the clump mass accretion rate is slightly superlinear with mass of the clump $\left(\dot{M} \sim M_{\text {clump }}^{5 / 4}\right)$. Maschberger et al. (2014) instead use a model of stochastic growth with a sublinear mass growth rate $\left(\dot{M} \sim M_{\text {sink }}^{2 / 3}\right)$. The exact nature of clump accretion and how it depends on the mass of the clump are still under debate; nevertheless, there is theoretical support for cloud-to-clump accretion.

There is also direct observational evidence for large-scale flows onto clumps and filaments. One of the most striking examples is a flow estimated at $2500 M_{\odot} \mathrm{Myr}^{-1}$ traced by $\mathrm{HCO}^{+} 1-0$ blue asymmetric line profiles along a filamentary complex into the central clump of the source SDC335.579-0.272 (SDC335; Peretto et al. 2013). The total mass of this region is $5500 M_{\odot}$, which would place this among the most massive clumps in our survey. Another example of inflow onto clumps is seen in the converging flows toward the DR21 filament complex with inflow rates of $1000 M_{\odot} \mathrm{Myr}^{-1}$ onto 4900 and $3300 M_{\odot}$ clumps from infall profiles of the $1-0$ transitions of $\mathrm{HCO}^{+}$and $\mathrm{CO}$ (Schneider et al. 2010). Analysis of velocity centroids can also reveal potential mass inflow onto clumps along filaments (see three examples from the IRDC survey in Tackenberg et al. 2014), although there can be a degeneracy between inflow and outflow in the interpretation (Henshaw et al. 2014). These regions are already forming protostars and display hub-filament geometries where filaments of dense gas appear to converge on the central hub, so they are not exact analogs to the massive starless clumps in BGPS; nevertheless, they show that clumps are deep gravitational potentials that draw surrounding material in.

The examples of large-scale inflow also extend to nearby lower-mass regions. For example, inflow signatures are seen in line profiles of HNC 1-0 toward the Serpens South region indicating mass flow rates of $130 \mathrm{M}_{\odot} \mathrm{Myr}^{-1}$ onto the filament complex and $28 M_{\odot} \mathrm{Myr}^{-1}$ onto the central cluster (Kirk et al. 2013; for a higher-resolution study of the same region, see Fernández-López et al. 2014). The central filament (L1495, B213) of the low-mass star-forming Taurus complex also shows direct evidence of a large-scale flow in CO 1-0 of 27-50 $M_{\odot} \mathrm{pc}^{-1} \mathrm{Myr}^{-1}$ perpendicular to the filament complex (Palmeirim et al. 2013). While these last two examples occur in lower-mass regions than the typical BGPS starless clumps, they show that flow rates of $10-100 M_{\odot} \mathrm{Myr}^{-1}$ are possible in lowmass regions. It therefore seems plausible that higher-mass inflow rates of a few $\times 10^{2} M_{\odot} \mathrm{Myr}^{-1}$ should be possible in the more massive starless clump regions seen in the BGPS.

\subsubsection{Different Starless Clump versus Protostellar Clump Lifetimes}

A third explanation for the observed mass difference is that the lifetime of the SCC phase decreases with increasing total clump mass in exact proportion to the fraction of SCCs at each mass. The shorter lifetime of massive clumps explains the lack of very massive $\left(M>10^{4} M_{\odot}\right)$ starless clump candidates. We estimate that the lifetime of this phase is less than $0.03 \mathrm{Myr}$ based on the $\mathrm{CH}_{3} \mathrm{OH}$ maser technique. Thus, protostellar clumps with $>10^{4} M_{\odot}$ require an alternative formation mechanism: the average mass growth from cloud-to-clump accretion is not sufficient to explain the lack of these massive SCCs. Using even a high mass infall rate of $\sim 10^{3} \mathrm{M}_{\odot} \mathrm{Myr}^{-1}$, the most massive clumps could only accrete a meager $\sim$ few $\times 10^{1} M_{\odot}$ over their $\sim$ few $\times 10^{4}$ yr starless-phase lifetimes.

The lifetime ratio of SCCs to protostellar clumps would have to decrease with increasing mass in proportion to the ratio $N_{\text {SCC }} / N_{\text {proto }}$ if lifetime arguments are the sole explanation for the observed median mass difference. The ratio of $N_{\mathrm{SCC}} / N_{\text {proto }}$ scales as $M^{-0.4}$ over the range $100 M_{\odot} \leqslant M \leqslant 1000 M_{\odot}$. The negative exponent implies that the lifetime scaling for SCCs is a steeper function of mass than the protostellar clump lifetime. 
The value of the exponent is insensitive to the lower bound of the mass between 100 and $300 M_{\odot}$. There is no known theoretical prediction of such a lifetime scaling between highmass SCCs and high-mass protostellar clumps.

With the current data, it is difficult to disentangle the relative importance of differing lifetimes for SCCs and protostellar clumps versus the importance of clump mass growth in explaining the median mass difference between SCCs and protostellar clumps. It is likely that both processes occur.

\section{SUMMARY}

We present a sample of 4683 molecular cloud clumps from the BGPS sorted by different observational indicators of star formation activity. We use a variety of Galactic plane surveys in a common overlap region between $10^{\circ}<\ell<65^{\circ}$ that include $70 \mu \mathrm{m}$ compact sources from Herschel Hi-GAL, midIR color-selected YSOs, $\mathrm{H}_{2} \mathrm{O}$ and $\mathrm{CH}_{3} \mathrm{OH}$ masers, and $\mathrm{UCH}$ II regions. We use $\mathrm{MC}$ random sampling to calculate the clump physical properties using a subsample of 1640 well-constrained clump DPDFs. We also present a catalog of 1663 clump $\mathrm{NH}_{3} T_{\mathrm{K}}$ measurements and $22 \mathrm{GHz} \mathrm{H}_{2} \mathrm{O}$ maser observations.

We find the following conclusions:

1. We identify a subsample of 2223 dense clumps with no indicators of star formation activity, representing the largest and most robust sample of SCCs from a blind survey to date.

2. We measure numerous increasing trends in median physical properties from starless candidates to the most extreme indicator of star formation, $\mathrm{UCH}$ II regions. These include $S_{1.1}^{\text {Total }}(\mathrm{Jy}), \quad \Omega^{\text {Total }} / \Omega^{\text {FWHM }}, \quad T_{\mathrm{K}}(\mathrm{K})$, $\Delta v\left(\mathrm{NH}_{3}\right) \quad\left(\mathrm{km} \mathrm{s}^{-1}\right), R_{\mathrm{eq}}^{\text {Total }}(\mathrm{pc}), \sum_{\mathrm{H}_{2}}^{\mathrm{FWHM}}\left(\mathrm{g} \mathrm{cm}^{-2}\right), n_{\mathrm{c}}$ $\left(\mathrm{cm}^{-3}\right)$, and $M_{\mathrm{H}_{2}}^{\text {Total }}\left(M_{\odot}\right)$.

3. Median-mass SCCs $\left(230 M_{\odot}\right)$ are capable of forming intermediate-mass protostars up to $6 M_{\odot}$ (assuming a Kroupa IMF and $\epsilon_{\mathrm{SF}}=0.3$ ), which are detectable by current mid- and far-infrared Galactic plane surveys. Mass is well correlated with radius for BGPS clumps, but the observed $M-R$ relationship lies above the Kauffmann $\&$ Pillai (2010) thresholds for massive star formation for only $10.8 \%$ of SCCs.

4. The average SCC freefall timescale is $\left\langle t_{\mathrm{ff}}\right\rangle=0.8 \mathrm{Myr}$. Using $\mathrm{CH}_{3} \mathrm{OH}$ masers and counting the number of SCCs to $\mathrm{CH}_{3} \mathrm{OH}$ maser containing clumps, we estimate that the lifetime of massive SCCs decreases as $\sim 0.37 \pm 0.08 \operatorname{Myr}\left(10^{3} M_{\odot} / M\right)$ for $M>10^{3} M_{\odot}$. There are no SCCs observed with $M>1.4 \times 10^{4} M_{\odot}$, indicating a very short lifetime of $<0.03 \mathrm{Myr}$. The majority of SCCs (median total mass $230 M_{\odot}$ ), however, have lifetimes that are longer than their average freefall timescale.

5. Virial parameters derived from $\mathrm{NH}_{3}$ show subvirial clumps with median $\alpha=0.7$. More than $75 \%$ of BGPS clumps are gravitationally bound $(\alpha<2)$. A medianproperty SCC would be in virial equilibrium if a modest $\sim 50 \mu \mathrm{G}$ magnetic field is present.

6. We find a median mass difference between the SCC and prostellar categories of $\Delta M_{\mathrm{H}_{2}}^{\text {Total }}=170-370 M_{\odot}$. It is unlikely that this mass difference can be solely explained by a population of SCCs that are incapable of forming detectable protostars in current Galactic plane surveys. If the observed mass difference is not due to a systematic increase in dust opacity of 1.7-2.5 from SCCs to protostellar clumps, then there are two likely explanations: (a) SCCs accrete mass from their surroundings and (b) that the lifetime of SCCs is proportionally longer than protostellar clumps at the same mass below $470 M_{\odot}$ and shorter than protostellar clumps at masses above $470 M_{\odot}$. If mass accretion is the sole explanation, then for an average clump freefall timescale, $\dot{M} \sim 200-440 M_{\odot} \mathrm{Myr}^{-1}$ is required. If the variation in SCC and protostellar clump lifetimes is the sole explanation, then their ratio of lifetimes should scale as $M^{-0.4}$ for $100 \leqslant M \leqslant 1000 M_{\odot}$. In reality, both processes likely occur, although it is not possible to disentangle them with the current data.

We sincerely thank the observatory staff at the Green Bank Telescope for their help during observing. We also sincerely thank Shari Breen for sharing coordinates to the MMB catalog between $20^{\circ}<\ell<60^{\circ}$ prior to publication. We would also like to thank Julia Kamenetzky and Kimberly Ward-Duong for many useful comments that benefited this paper. B.E.S. was supported by the National Science Foundation Graduate Research Fellowship under grant No. DGE-1143953. Y.L.S. and B.E.S. were supported in part by the NSF Grants AST-1008577 and AST1410190. N.J.E. was supported by NSF grant AST-1109116 to the University of Texas at Austin. E.W.R. was supported by a Discovery Grant from NSERC of Canada.

\section{REFERENCES}

Aguirre, J. E., Ginsburg, G., Dunham, M., et al. 2011, ApJS, 192, 4 André, P., Belloche, A., Motte, F., \& Peretto, N. 2007, A\&A, 472, 519

André, P., Di Francesco, J., Ward-Thompson, D., et al. 2014, in Protostars and Planets VI, 27

Bally, J., Anderson, L., Battersby, C., et al. 2010, A\&A, 518, L90

Battersby, C. 2013, PhD thesis, Univ. Colorado at Boulder

Battersby, C., Bally, J., Dunham, M., et al. 2014, ApJ, 786, 116

Battersby, C., Bally, J., Jackson, J. M., et al. 2010, ApJ, 721, 222

Battersby, C., Bally, J., Ginsburg, A., et al. 2011, A\&A, 535, A128

Benjamin, R. A., Churchwell, E., Babler, B. L., et al. 2003, PASP, 115, 953

Bertoldi, F., \& McKee, C. F. 1992, ApJ, 395, 140

Beuther, H., Schilke, P., Menten, K. M., et al. 2002, ApJ, 566, 945

Boissier, S., \& Prantzos, N. 1999, MNRAS, 307, 857

Bonnell, I. A., Bate, M. R., Clarke, C. J., \& Pringle, J. E. 2001, MNRAS, 323, 785

Bonnell, I. A., Larson, R. B., \& Zinnecker, H. 2007, in Protostars and Planets V, ed. V. B. Reipurth, D. Jewitt, \& K. Keil (Tuscon, AZ: Univ. of Arizona Press), 149

Bonnor, W. B. 1956, MNRAS, 116, 351

Bontemps, S., Andre, P., Terebey, S., \& Cabrit, S. 1996, A\&A, 311, 858

Bontemps, S., Motte, F., Csengeri, T., \& Schneider, N. 2010, A\&A, 524, A18

Breen, S. L., Ellingsen, S. P., Caswell, J. L., et al. 2012a, MNRAS, 426, 2189

Breen, S. L., Ellingsen, S. P., Caswell, J. L., et al. 2012b, MNRAS, 421, 1703

Breen, S. L., Ellingsen, S. P., Contreras, Y., et al. 2013, MNRAS, 435, 524

Breen, S. L., Ellingsen, S. P., Caswell, J. L., et al. 2014, MNRAS, 438, 3368

Breen, S. L., Fuller, G. A., Caswell, J. L., et al. 2015, MNRAS, 450, 4109

Bressert, E., Ginsburg, A., Bally, J., et al. 2012, ApJL, 758, L28

Carey, S. J., Noriega-Crespo, A., Mizuno, D. R., et al. 2009, PASP, 121, 76

Chambers, E. T., Jackson, J. M., Rathborne, J. M., \& Simon, R. 2009, ApJS, 181,360

Chen, X., Gan, C.-G., Ellingsen, S. P., et al. 2013, ApJS, 206, 9

Chomiuk, L., \& Povich, M. S. 2011, AJ, 142, 197

Churchwell, E., Babler, B. L., Meade, M. R., et al. 2009, PASP, 121, 213

Contreras, Y., Schuller, F., Urquhart, J. S., et al. 2013, A\&A, 549, A45

Crutcher, R. M. 2012, ARA\&A, 50, 29

Csengeri, T., Urquhart, J. S., Schuller, F., et al. 2014, A\&A, 565, A75

Cyganowski, C. J., Whitney, B. A., Holden, E., et al. 2008, AJ, 136, 2391

Danby, G., Flower, D. R., Valiron, P., Schilke, P., \& Walmsley, C. M. 1988, MNRAS, 235, 229

Davies, B., Hoare, M. G., Lumsden, S. L., et al. 2011, MNRAS, 416, 972 
di Francesco, J., Evans, N. J., II, Caselli, P., et al. 2007, in Protostars and Planets V, ed. V. B. Reipurth, D. Jewitt, \& K. Keil (Tuscon, AZ: Univ. of Arizona Press), 17

Duarte-Cabral, A., Bontemps, S., Motte, F., et al. 2013, A\&A, 558, A125

Dunham, M. K., Robitaille, T. P., Evans, N. J., II, et al. 2011a, ApJ, 731, 90

Dunham, M. K., Rosolowsky, E., Evans, N. J., II, Cyganowski, C., \& Urquhart, J. S. 2011b, ApJ, 741, 110

Dunham, M. K., Rosolowsky, E., Evans, N. J., et al. 2010, ApJ, 717, 1157

Dunham, M. M., Stutz, A. M., Allen, L. E., et al. 2014, in Protostars and Planets VI, ed. H. Beother et al. (Tuscon, AZ: Univ. of Arizona Press), 195 Ebert, R. 1955, ZAp, 37, 217

Ellsworth-Bowers, T. P., Rosolowsky, E., Glenn, J., et al. 2015, ApJ, 799, 29

Ellsworth-Bowers, T. P., Glenn, J., Rosolowsky, E., et al. 2013, ApJ, 770, 39

Ellsworth-Bowers, T. P., Glenn, J., Riley, A., et al. 2015, ApJ, 805, 157

Evans, N. J., Shirley, Y. L., Mueller, K. E., \& Knez, C. 2002, in ASP Conf. Ser. 267, Hot Star Workshop III: The Earliest Phases of Massive Star Birth, ed. P. Crowther (San Francisco, CA: ASP), 17

Fernández-López, M., Arce, H. G., Looney, L., et al. 2014, ApJL, 790, L19

Furuya, R. S., Kitamura, Y., Wootten, A., Claussen, M. J., \& Kawabe, R. 2003, ApJS, 144, 71

Ginsburg, A., Bressert, E., Bally, J., \& Battersby, C. 2012, ApJL, 758, L29

Ginsburg, A., Glenn, J., Rosolowsky, E., et al. 2013, ApJS, 208, 14

Goldsmith, P. F. 2001, ApJ, 557, 736

Greene, T. P., Wilking, B. A., Andre, P., Young, E. T., \& Lada, C. J. 1994, ApJ, 434, 614

Gutermuth, R. A., \& Heyer, M. 2015, AJ, 149, 64

Guzmán, A. E., Sanhueza, P., Contreras, Y., et al. 2015, ApJ, 815, 130

Habing, H. J. 1968, BAN, 19, 421

Heiderman, A., Evans, N. J., II, Allen, L. E., Huard, T., \& Heyer, M. 2010, ApJ, 723, 1019

Henshaw, J. D., Caselli, P., Fontani, F., Jiménez-Serra, I., \& Tan, J. C. 2014, MNRAS, 440, 2860

Heyer, M., Krawczyk, C., Duval, J., \& Jackson, J. M. 2009, ApJ, 699, 1092

Ho, P. T. P., \& Townes, C. H. 1983, ARA\&A, 21, 239

Hoare, M. G., Purcell, C. R., Churchwell, E. B., et al. 2012, PASP, 124, 939

Hollenbach, D., Elitzur, M., \& McKee, C. F. 2013, ApJ, 773, 70

Hoq, S., Jackson, J. M., Foster, J. B., et al. 2013, ApJ, 777, 157

Huchra, J. P., \& Geller, M. J. 1982, ApJ, 257, 423

Imai, H., Obara, K., Diamond, P. J., Omodaka, T., \& Sasao, T. 2002, Natur, 417, 829

Jackson, J. M., Rathborne, J. M., Shah, R. Y., et al. 2006, ApJS, 163, 145

Jørgensen, J. K., Johnstone, D., van Dishoeck, E. F., \& Doty, S. D. 2006, A\&A, 449, 609

Kauffmann, J., \& Pillai, T. 2010, ApJL, 723, L7

Kauffmann, J., Pillai, T., \& Goldsmith, P. F. 2013, ApJ, 779, 185

Kirk, H., Myers, P. C., Bourke, T. L., et al. 2013, ApJ, 766, 115

Kroupa, P. 2001, MNRAS, 322, 231

Krumholz, M. R., Klein, R. I., \& McKee, C. F. 2007, ApJ, 656, 959

Krumholz, M. R., \& McKee, C. F. 2008, Natur, 451, 1082

Krumholz, M. R., \& Thompson, T. A. 2012, ApJ, 760, 155

Kuiper, R., Klahr, H., Beuther, H., \& Henning, T. 2010, ApJ, 722, 1556

Lada, C. J., \& Lada, E. A. 2003, ARA\&A, 41, 57

Lada, C. J., Lombardi, M., \& Alves, J. F. 2010, ApJ, 724, 687

Larson, R. B. 1981, MNRAS, 194, 809

Launhardt, R., Stutz, A. M., Schmiedeke, A., et al. 2013, A\&A, 551, A98

Longmore, S. N., Kruijssen, J. M. D., Bastian, N., et al. 2014, in Protostars and Planets VI, ed. H. Beother et al. (Tuscon, AZ: Univ. of Arizona Press), 291

Lumsden, S. L., Hoare, M. G., Oudmaijer, R. D., \& Richards, D. 2002, MNRAS, 336, 621

Lumsden, S. L., Hoare, M. G., Urquhart, J. S., et al. 2013, ApJS, 208, 11

Malinen, J., Juvela, M., Collins, D. C., Lunttila, T., \& Padoan, P. 2011, A\&A, 530, A101

Maschberger, T., Bonnell, I. A., Clarke, C. J., \& Moraux, E. 2014, MNRAS, 439, 234

McKee, C. F., \& Ostriker, E. C. 2007, ARA\&A, 45, 565

McKee, C. F., \& Tan, J. C. 2003, ApJ, 585, 850

Merello, M., Evans, N. J., II, Shirley, Y. L., et al. 2015, ApJS, 218, 1

Molinari, S., Schisano, E., Faustini, F., et al. 2011, A\&A, 530, A133

Molinari, S., Swinyard, B., Bally, J., et al. 2010, PASP, 122, 314

Morales, E. F. E., Wyrowski, F., Schuller, F., \& Menten, K. M. 2013, A\&A, 560, A76

Mueller, K. E., Shirley, Y. L., Evans, N. J., II, \& Jacobson, H. R. 2002, ApJS, 143,469

Murray, N., \& Chang, P. 2012, ApJ, 746, 75
Ossenkopf, V., \& Henning, T. 1994, A\&A, 291, 943

Palmeirim, P., André, P., Kirk, J., et al. 2013, A\&A, 550, A38

Pandian, J. D., Goldsmith, P. F., \& Deshpande, A. A. 2007, ApJ, 656, 255

Pandian, J. D., Momjian, E., Xu, Y., Menten, K. M., \& Goldsmith, P. F. 2011 ApJ, 730, 55

Pattle, K., Ward-Thompson, D., Kirk, J. M., et al. 2015, MNRAS, 450, 1094 Peretto, N., \& Fuller, G. A. 2009, A\&A, 505, 405

Peretto, N., Fuller, G. A., Duarte-Cabral, A., et al. 2013, A\&A, 555, A112

Pestalozzi, M. R., Minier, V., \& Booth, R. S. 2005, A\&A, 432, 737

Pillai, T., Kauffmann, J., Tan, J. C., et al. 2015, ApJ, 799, 74

Purcell, C. R., Hoare, M. G., Cotton, W. D., et al. 2013, ApJS, 205, 1

Ragan, S. E., Henning, T., \& Beuther, H. 2013, A\&A, 559, A79

Rebull, L. M., Stapelfeldt, K. R., Evans, N. J., II, et al. 2007, ApJS, 171, 447

Reid, M. J., Menten, K. M., Brunthaler, A., et al. 2014, ApJ, 783, 130

Robitaille, T. P., Whitney, B. A., Indebetouw, R., \& Wood, K. 2007, ApJS, 169,328

Robitaille, T. P., Whitney, B. A., Indebetouw, R., Wood, K., \& Denzmore, P 2006, ApJS, 167, 256

Robitaille, T. P., Meade, M. R., Babler, B. L., et al. 2008, AJ, 136, 2413

Roman-Duval, J., Jackson, J. M., Heyer, M., et al. 2009, ApJ, 699, 1153

Roman-Duval, J., Jackson, J. M., Heyer, M., Rathborne, J., \& Simon, R. 2010 , ApJ, 723, 492

Rosolowsky, E. W., Pineda, J. E., Foster, J. B., et al. 2008, ApJS, 175, 509

Rosolowsky, E., Dunham, M. K., Ginsburg, A., et al. 2010, ApJS, 188, 123

Schlingman, W. M., Shirley, Y. L., Schenk, D. E., et al. 2011, ApJS, 195, 14

Schneider, N., Csengeri, T., Bontemps, S., et al. 2010, A\&A, 520, A49

Schuller, F., Menten, K. M., Contreras, Y., et al. 2009, A\&A, 504, 415

Scoville, N. Z., Yun, M. S., Sanders, D. B., Clemens, D. P., \& Waller, W. H. 1987, ApJS, 63, 821

Shirley, Y. L. 2015, PASP, 127, 299

Shirley, Y. L., Evans, N. J., II, Young, K. E., Knez, C., \& Jaffe, D. T. 2003 ApJS, 149, 375

Shirley, Y. L., Huard, T. L., Pontoppidan, K. M., et al. 2011, ApJ, 728, 143 Shirley, Y. L., Nordhaus, M. K., Grcevich, J. M., et al. 2005, ApJ, 632, 982

Shirley, Y. L., Ellsworth-Bowers, T. P., Svoboda, B., et al. 2013, ApJS, 209, 2 Smith, R. J., Longmore, S., \& Bonnell, I. 2009, MNRAS, 400, 1775

Solomon, P. M., Rivolo, A. R., Barrett, J., \& Yahil, A. 1987, ApJ, 319, 730

Tackenberg, J., Beuther, H., Henning, T., et al. 2012, A\&A, 540, A113

Tackenberg, J., Beuther, H., Henning, T., et al. 2014, A\&A, 565, A101

Tafalla, M., Myers, P. C., Caselli, P., \& Walmsley, C. M. 2004, Ap\&SS, 292, 347

Tan, J. C., Beltran, M. T., Caselli, P., et al. 2014, in Protostars and Planets VI, 149

Traficante, A., Fuller, G. A., Peretto, N., Pineda, J. E., \& Molinari, S. 2015a MNRAS, 451, 3089

Traficante, A., Fuller, G. A., Pineda, J. E., \& Pezzuto, S. 2015b, A\&A, 574, A119

Traficante, A., Calzoletti, L., Veneziani, M., et al. 2011, MNRAS, 416, 2932

Urquhart, J. S., Morgan, L. K., Figura, C. C., et al. 2011, MNRAS, 418, 1689

Urquhart, J. S., Moore, T. J. T., Schuller, F., et al. 2013a, MNRAS, 431, 1752

Urquhart, J. S., Thompson, M. A., Moore, T. J. T., et al. 2013b, MNRAS, 435,400

Urquhart, J. S., Moore, T. J. T., Csengeri, T., et al. 2014, MNRAS, 443, 1555

Urquhart, J. S., Moore, T. J. T., Menten, K. M., et al. 2015, MNRAS, 446, 3461

Valdettaro, R., Palla, F., Brand, J., et al. 2001, A\&A, 368, 845

van der Walt, J. 2005, MNRAS, 360, 153

Vázquez-Semadeni, E., Gómez, G. C., Jappsen, A.-K., Ballesteros-Paredes, J., \& Klessen, R. S. 2009, ApJ, 707, 1023

Walsh, A. J., Purcell, C. R., Longmore, S. N., et al. 2014, MNRAS, 442, 2240

Walsh, A. J., Breen, S. L., Britton, T., et al. 2011, MNRAS, 416, 1764

Ward-Thompson, D., Scott, P. F., Hills, R. E., \& Andre, P. 1994, MNRAS, 268,276

Wienen, M., Wyrowski, F., Schuller, F., et al. 2012, A\&A, 544, A146

Wienen, M., Wyrowski, F., Menten, K. M., et al. 2015, A\&A, 579, A91

Wilcock, L. A., Ward-Thompson, D., Kirk, J. M., et al. 2012, MNRAS, 424, 716

Wolfire, M. G., McKee, C. F., Hollenbach, D., \& Tielens, A. G. G. M. 2003, ApJ, 587, 278

Young, K. E., Lee, J.-E., Evans, N. J., II, Goldsmith, P. F., \& Doty, S. D. 2004 ApJ, 614, 252

Zuckerman, B., \& Evans, N. J., II 1974, ApJL, 192, L149 